Danmarks Geologiske Undersøgelse. II. Række. Nr. 68.

\title{
Chemical Analyses \\ of Igneous and Metamorphic Rocks \\ of \\ Denmark, the Faeroes and Greenland.
}

(To and including 1940)

\author{
Collected and Described \\ by \\ Sole Munck and Arne Noe-Nygaard.
}

\author{
I Kommission hos \\ C. A. Reitzels Forlag \\ Axel Sandal \\ København 1942.
}

Pris: $4 \mathrm{Kr}$. 
Danmarks Geologiske Undersøgelse.

II. Række. Nr. 68.

\section{Chemical Analyses of Igneous and Metamorphic Rocks \\ of \\ Denmark, the Faeroes and Greenland.}

(To and including 1940)

Collected and Described

by

Sole Munck and Arne Noe-Nygaard

I Kommission hos

C. A. Reitzels Forlag

Axel Sandal

København

1942 . 



\section{Contents.}

Page

List of Chemical Analyses of Rocks .............. 10

Denmark ............................. 10

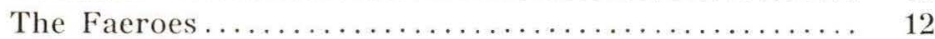

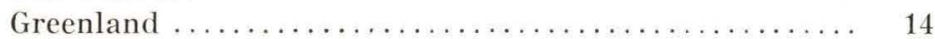

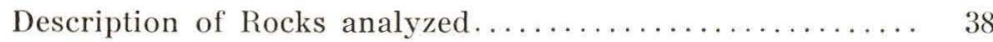

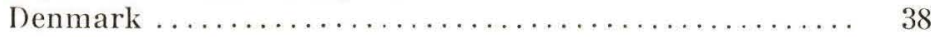

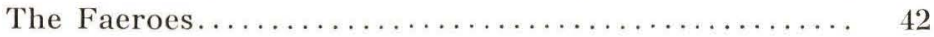

Greenland ............................ 46

Appendix No. 1. Analyses of Telluric Iron............ 82

Appendix No. 2. Analyses of Meteoric Iron ............. 86

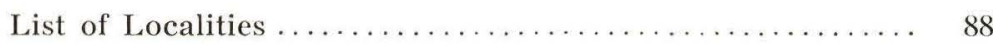

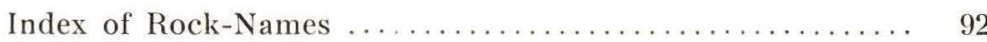

Literature . . . . . . . . . . 96

Maps ............................... 100 


\section{Preface.}

$\mathrm{T}$ he past ten years or so have seen the publication of collections of chemical rock analyses which, as a result of their clear form of set-up, in many ways faciliate the comparative study of the chemistry of the rocks and their mutual relationships. Among these publications there are: P. Niggli, F. DE Quervain \& R. U. Winterthalter: Chemismus schweizerischer Gesteine. Bern 1930, and the analyses published by the Geological Survey of Great Britain: Chemical Analyses of Igneous Rocks, Metamorphic Rocks and Minerals. London 1931. Similar publications are available from two neighbouring countries, i. e. from Sweden: W. Larsson: Chemical Analyses of Swedish Rocks (Bull. Geol. Inst., Uppsala 1932) and from Finland: L. LokkA: Neuere Chemische Analysen von Finnischen Gesteinen (Bull. Comm. Geol. de Finlande No. 105. Helsingfors 1934).

In now issuing a collection of analyses comprising Denmark, the Faeroes and Greenland, we do so for the purpose of supplying the need we have felt in not possessing a collection of this kind for the regions named. The framework around the present work is a file of rock analyses made during the course of some years.

We have not thought the material comprehensive enough to warrant a calculation and discussion of the analyses like that published by W. LARsson for Sweden; for Greenland the analysis-material is somewhat sporadic, even if certain regions there are well represented, for instance the Julianehaab district (Ussing), the Kangerdlugssuaq area (WAGER \& DEER), and parts of the West Greenland basalt formation; in this case, however, some of the analyses are rather old.

As a consequence, this collection has been treated more in a manner reminiscent of that applied to the British analyses. In that collection the analyses were got together by E. M. GuPPy, and to each analysis is appended a brief petrographic description of the analyzed rock by H. H. Thomas. As in that case the rock material has been microscoped by one and the same worker, its presentation is homogeneous in character. We have been unable to emulate that example, as only 
a small part of the rock material has been available to us; we have therefore chosen the expedient of first listing all the chemical analyses in tabular form, and then presenting a short characteristic of the rocks, not on the basis of a microscopical examination but on that of the authors' own descriptions. It is evident that the descriptions of the rocks, which are kept as closely to those of the authors as possible, by this means will be somewhat less uniform: in some cases more explicit, in others rather summary; and as some of the descriptions quoted are rather old, their value to the reader today may be limited.

The chief importance of these analyses lies, in our opinion, in the fact that we have endeavoured to gather the available material into one place, in perspicuous form and with all necessary references to the literature and the geographical situation. The accompanying description of the rocks is intended as a primary orientation as regards their character and mineral composition. A reader requiring greater detail should have no difficulty in finding the original literature.

The analysis tables list the analyses according to the falling $\mathrm{SiO}_{2}$ content. Igneous rocks are shown in ordinary figures, metamorphic rocks in italics, pyroclastics are marked with an asterisk behind the figure $^{1}$ ). The enumeration in the table is the same as that employed in the rock descriptions. In the list of Greenland analyses there is a column more than in the lists of Danish and Faeroese analyses, marked »district«; in this column the four main parts of Greenland from which the rock came are designated by NW, SW, NE and SE (compare the maps at the end of the book) ${ }^{2}$ ). The years in which the analyses were published are given in the table, to show at a glance how old they are; as the total number of rock analyses given in the list is only little more than two hundred, no division into separate groups after completeness and accuracy was made; this means that the table contains both "superior « and »inferior « analyses in the sense of Washington.

In the table the column "Sum" indicates the value arrived at by adding up the values stated; where this sum differs from that stated by the author, the latter is given below in brackets. This is done because of the possibility that the sum may be correct, in which case an error must have crept into one of the individual values.

In the columns marked $» \mathrm{H}_{2} \mathrm{O}^{+}$" and $\mathrm{H}_{2} \mathrm{O}^{-}$" the rule followed is that where no distinction is made in the analysis between absorbed

\footnotetext{
1) The two analyses marked 28 a and 126 a were entered in the table after it had been completed; in the first tabulation they had escaped our attention.

$\left.{ }^{2}\right)$ Division lines of the districts are: $\mathrm{N}-\mathrm{S}$ line from cape Farvel to mouth of de Long Fjord, and E-W line from Scoresby Sound to south of Disko through Jakobshavn icefjord.
} 
water and combined water, the water percentage is shown in the first column $\left(\mathrm{H}_{2} \mathrm{O}+\right)$.

In the rock descriptions the name and age of the rock are given first. For some of the East-Greenland rocks the term »metamorphic complex" is employed, as in the available literature there is some divergence of opinion on the subject of pre-Cambrian/Caledonian; in those cases where the author's own opinion is clearly expressed his term is shown in brackets. Where a subsequent examination reverses a previous age determination, this is specially indicated.

The rock names used are those employed by the various authors.

After name and age follows the geographical locality where it was found, then a reference to the source, showing the page, and finally the description itself. Where the author has given the rocks special numbers - museum numbers or field labels - this is shown in brackets together with the literature reference.

In cases where old and incomplete descriptions from a region preponderate, more weight is attached to the few more modern descriptions from that area, e. g. the Faeroes. Where the nature of the rock or the character of the description was of such a kind that a summary was difficult to give, the description has been written in greater detail and kept as close to the original as possible. It being the fact that the authors' own descriptions of the rocks have been used as the basis for our account, it will be easy to find inconsistencies from rock to rock, just as it will be observed that different terms are used promiscuously for the same things, for example idiomorphic and automorphic, etc.

The spelling of the Greenland place names used in this paper may at the first look seem rather haphazard; although inconsistences no doubt occur the following rules have been used. The forms employed by Ussing (50) have been preserved, since a modernization of local names already established in a given form in the literature was considered unfortunate. Other locality names in West Greenland have been modernized in accordance with the spelling used in the new maps carried out by the Geodetic Institute of Copenhagen. In East Greenland in spite of the Danish spelling of $\varnothing$, Kap, Havn etc. in the maps (also in this paper) the English forms island, cape, harbour etc. have been used, because also here a considerable number of place names were first used in this form.

Apart from the rock analyses proper, we have included as Appendix No. I the available material concerning the telluric iron from the West-Greenland basalt formation. The analyses are arranged in tabular form and are unaccompanied by any separate description. The iron is arranged according to locality, beginning with oldest. Although 
the iron discussion now belongs to the past, and the problems have been clarified through the new researches of BENEDICKS and LöFQuisT in 1940, a collection of analyses from Greenland could not, we consider, be called complete unless the iron analyses were included. Together with the iron is given an analysis, no. 256 of "pyrite nickelifère from Igdlokunguaq (cf. lit. 47).

Again in the iron table we have included the year of analysis so that its age may be seen at a glance. We have made the collection as complete as possible, well aware that some of the analyses are scarcely of much value today and that the technique employed from time to time must have had a bearing on the results. Our reason for collecting them all in a more or less uniform manner is that these iron analyses have been published in so many different places, a great part of them in foreign journals.

Analyses made on acid-treated or heat-treated material, whereby essential components were affected or removed, are not included (for instance analyses by CL. WinkLER).

The analyses of Greenland meteoric iron are given in the short Appendix II.

As already stated, this work was begun on the material contained in a file covering a period of some years. We divided the work between us, SolE MUNCK being mainly responsible for the analyses from Bornholm (Denmark) and West Greenland and ARne NoE-NYGAARD for the rest of Denmark, the Faerocs and East Greenland. The collection comprises analyses up to and including 1940.

Although we believe that the collection is complete in all essentials, it would be strange if an analysis or two had not escaped our attention; and even if we have taken pains to avoid error, it is obvious that an incorrect value may have been included inadvertently in our table. We shall therefore be grateful for any correction communicated to us by colleagues making use of our collection.

As most of the petrographic literature, and especially that from Greenland, has been issued in English, we have continued in that language and translated the other descriptions from Danish, French or German. The English manuscript has been corrected by Mr. W. E. Calvert.

Copenhagen/Charlottenlund, November the 3rd 1941.

Sole Munck and Arne Noe-NygaARd. 
Denmark.

\section{List of Chemical}

\begin{tabular}{|c|c|c|c|c|c|c|c|c|c|c|c|}
\hline No. & Rock Name & Locality & Year & $\mathrm{SiO}_{2}$ & $\mathrm{TiO}_{2}$ & $\mathrm{Al}_{2} \mathrm{O}_{3}$ & $\mathrm{Fe}_{2} \mathrm{O}_{3}$ & $\mathrm{FeO}$ & $\mathrm{MnO}$ & $\mathrm{MgO}$ & $\mathrm{CaO}$ \\
\hline 1 & Alminding granite ${ }^{*}$ ) & Bjergbakke & $\begin{array}{c}1932 \\
(1934)\end{array}$ & 74.17 & 0.40 & 12.89 & 0.69 & 1.07 & tr. & 0.28 & 1.14 \\
\hline 2 & Hammer granite & Hammeren & 1913 & 73.77 & 0.32 & 11.97 & 1.84 & 0.78 & & 0.23 & 1.10 \\
\hline 3 & Hammer granite & Hammeren & $\begin{array}{c}1928 \\
(1932)\end{array}$ & 72.82 & 0.63 & 13.42 & 2.33 & & 0.24 & 0.13 & 1.61 \\
\hline 4 & Granite & Allinge & 1914 & 72.96 & & $\left.13.40^{1}\right)$ & $\left.3.40^{2}\right)$ & & & $\left.0.52^{3}\right)$ & 1.32 \\
\hline 5 & Svaneke granite ${ }^{*}$ ) & Ibs Kirke & $\begin{array}{c}1932 \\
(1934)\end{array}$ & 69.06 & 0.65 & 14.15 & 1.27 & 2.44 & tr. & 0.82 & 2.02 \\
\hline 6 & Vang granite & $\begin{array}{l}\text { "At pegmatite ( Klon- } \\
\text { dyke»), Vang }\end{array}$ & 1913 & 69.01 & 0.97 & 12.16 & 2.07 & 2.40 & 0.06 & 0.93 & 2.28 \\
\hline 7 & Vang granite & Rødkløv & 1913 & 66.99 & 0.71 & 13.00 & 2.98 & 2.23 & 0.11 & 0.65 & 2.64 \\
\hline 8 & Ronne granite & Klippegaard & $\begin{array}{c}1890 \\
(1909)\end{array}$ & 66.11 & & 16.12 & 4.93 & 4.87 & & 1.92 & 0.39 \\
\hline 9 & Paradisbakke granite & Paradisbakkerne & 1913 & 65.40 & 1.01 & 14.73 & 1.14 & 2.92 & 0.06 & 1.02 & 2.78 \\
\hline 10 & Rønne granite & Klippegaard & $\begin{array}{c}1932 \\
(1934)\end{array}$ & 65.39 & 0.28 & 14.32 & 7.85 & & & 1.12 & 3.53 \\
\hline 11 & Rønne granite & Rønne area & 1913 & 64.49 & 1.22 & 13.67 & 1.63 & 4.42 & 0.14 & 1.38 & 3.12 \\
\hline 12 & Rønne granite & "Atpegmatite«Rønne area & 1913 & 64.13 & 0.99 & 13.57 & 2.40 & 4.11 & 0.06 & 1.45 & 2.91 \\
\hline $13^{*}$ & Ash-1ayer +19 & Ejerslev, Mors & 1918 & $\begin{array}{l}63.60 \\
67.27\end{array}$ & $\begin{array}{l}1.33 \\
1.41\end{array}$ & $\begin{array}{l}13.06 \\
13.81\end{array}$ & $\begin{array}{l}1.83 \\
1.94\end{array}$ & $\begin{array}{l}2.83 \\
2.99\end{array}$ & $\begin{array}{l}\operatorname{tr} \\
\operatorname{tr}\end{array}$ & $\begin{array}{l}0.37 \\
0.39\end{array}$ & $\begin{array}{l}1.56 \\
1.65\end{array}$ \\
\hline $14^{*}$ & Ash-layer +19 & Fur & 1940 & $\begin{array}{l}63.56 \\
70.18\end{array}$ & $\begin{array}{l}1.11 \\
1.23\end{array}$ & $\begin{array}{l}12.30 \\
13.58\end{array}$ & $\begin{array}{l}2.46 \\
2.72\end{array}$ & $\begin{array}{l}2.48 \\
2.74\end{array}$ & $\begin{array}{l}0.10 \\
0.11\end{array}$ & $\begin{array}{l}0.70 \\
0.77\end{array}$ & $\begin{array}{l}1.58 \\
1.18\end{array}$ \\
\hline 15 & Rønne granite & $\begin{array}{l}800 \mathrm{~m} \text {. east of St. Alme- } \\
\text { gaard }\end{array}$ & $\begin{array}{c}1932 \\
(1934)\end{array}$ & 63.54 & 1.15 & 14.23 & 3.56 & 3.40 & 0.24 & 0.91 & 3.27 \\
\hline $16^{*}$ & Ash-làyer -17 & Limfjord area & 1918 & $\begin{array}{l}52.28 \\
63.6\end{array}$ & $\begin{array}{l}2.07 \\
2.6\end{array}$ & $\begin{array}{l}15.50 \\
18.8\end{array}$ & $\begin{array}{l}3.63 \\
4.4\end{array}$ & $\begin{array}{l}0.50 \\
0.6\end{array}$ & $\begin{array}{l}\operatorname{tr} \\
\operatorname{tr}\end{array}$ & $\begin{array}{l}\operatorname{tr} \\
\operatorname{tr}\end{array}$ & $\begin{array}{l}2.74 \\
3.3\end{array}$ \\
\hline $17^{*}$ & Ash-layer -12 & Hanklit & 1918 & $\begin{array}{l}50.42 \\
52.12\end{array}$ & $\begin{array}{l}6.26 \\
6.47\end{array}$ & $\begin{array}{l}14.09 \\
14.57\end{array}$ & $\begin{array}{l}2.72 \\
2.81\end{array}$ & $\begin{array}{l}6.40 \\
6.61\end{array}$ & $\begin{array}{l}\operatorname{tr} \\
\operatorname{tr}\end{array}$ & $\begin{array}{l}2.80 \\
2.90\end{array}$ & $\begin{array}{l}7.62 \\
7.88\end{array}$ \\
\hline $18 \%$ & Ash-layer +19 & Helgenas, Djursland & 1940 & $\begin{array}{l}50.34 \\
70.63\end{array}$ & $\begin{array}{l}1.18 \\
1.65\end{array}$ & $\begin{array}{l}10.56 \\
14.81\end{array}$ & $\begin{array}{r}11.25 \\
2.39\end{array}$ & $\begin{array}{l}0.54 \\
0.76\end{array}$ & $\begin{array}{l}0.03 \\
0.04\end{array}$ & $\begin{array}{l}4.31 \\
6.05\end{array}$ & $\begin{array}{l}0.62 \\
0.15\end{array}$ \\
\hline
\end{tabular}

*) In D. G. U. V Række no. 4 two analyses, one of Alminding, one of Svaneke granite were published on p. 18; they have later been deleted by the author (cf. 8 p. 21). 
Denmark.

\begin{tabular}{|c|c|c|c|c|c|c|c|c|c|c|c|}
\hline $\mathrm{K}_{2} \mathrm{O}$ & $\mathrm{P}_{2} \mathrm{O}_{5}$ & $\mathrm{H}_{2} \mathrm{O}^{+}$ & $\mathrm{H}_{2} \mathrm{O}^{-}$ & $\mathrm{CO}_{2}$ & Inclusive & Sum & Sp.gr. & Analyst & Published by & Notes & No. \\
\hline 5.72 & 0.04 & 0.08 & & 0.19 & & 99.85 & 2.641 & Chr. Detlefsen & K. Callisen & & 1 \\
\hline 5.61 & & 0.49 & 0.65 & & & 99.51 & & M. Dittrich & G. Kalb & & 2 \\
\hline 5.47 & & & & & $\begin{array}{l}\text { Loss on } \\
\text { ignition : } 0.21\end{array}$ & 100.11 & 2.653 & Chr. Detlefsen & K. Callisen & & 3 \\
\hline 5.32 & 0.15 & 0.34 & 0.03 & & & 99.91 & & K. Rørdam & K. Rørdam & $\begin{array}{l}\text { 1) Including } \\
\mathrm{TiO}_{2} \text {. } \\
\text { 2) State of oxy- } \\
\text { dation not de- } \\
\text { termined. } \\
{ }^{3} \text { ) Trace of MnO. }\end{array}$ & 4 \\
\hline 5.12 & 0.12 & 0.12 & & 0.15 & & 99.61 & 2.706 & Chr. Detlefsen & K. Callisen & & 5 \\
\hline 4.81 & 0.11 & 0.42 & 0.44 & 0.42 & & 99.73 & & M. Dittrich & G. Kalb & & 6 \\
\hline 4.39 & 0.57 & 0.70 & 0.78 & & & 99.03 & 2.705 & M. Dittrich & G. Kalb & & 7 \\
\hline 1.26 & 0.27 & & & & $\begin{array}{l}\text { F:tr. } \\
\text { L. o. ign.: } 0.5 .5\end{array}$ & 100.59 & & K. Rørdam & K. Rordam & & 8 \\
\hline 4.31 & 0.19 & 1.58 & 0.55 & 0.68 & & 99.91 & 2.765 & M. Dittrich & (i. Kall) & & 9 \\
\hline 4.40 & & & & & L. o. ign.: 0.13 & 100.66 & 2.758 & Chr. Detlefsen & K. Callisen & & 10 \\
\hline 4.40 & 0.58 & 1.11 & 0.46 & & & 100.19 & & M. Dittrich & G. Kalb & & 11 \\
\hline 3.46 & 0.56 & 1.45 & 0.37 & 0.59 & & 100.36 & & M. Dittrich & G. Kalb & & 12 \\
\hline 2.80 & 0.27 & 5.24 & 1.61 & & & 101.39 & & Chr. Winther & O. B. Boggild & & $13^{*}$ \\
\hline 2.96 & 0.29 & - & - & & & 100.00 & & & & $\begin{array}{l}\text { reduced values } \\
\text { Rolf Norin }\end{array}$ & \\
\hline $\begin{array}{l}3.50 \\
3.86\end{array}$ & $\begin{array}{l}0.08 \\
0.09\end{array}$ & $\frac{6.81}{-}$ & $\frac{0.65}{-}$ & $\stackrel{0.40}{-}$ & & $\begin{array}{r}98.94 \\
100.00\end{array}$ & & Rolf Norin & Rolf Norin & r. v. R. X. & $14^{*}$ \\
\hline 6.09 & 0.23 & & & & L. o. ign.: 0.27 & 100.01 & 2.744 & Chr. Detlefsen & K. Callisen & & 15 \\
\hline 1.68 & & 9.04 & 9.66 & & & 100.89 & & Chr. Winther & O. B. Bøggild & & $16^{*}$ \\
\hline 2.1 & & - & - & & & 100.00 & & & 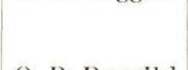 & $\begin{array}{l}\text { r.v.O. B. Bog- } \\
\text { gild }\end{array}$ & \\
\hline $\begin{array}{l}1.10 \\
1.14\end{array}$ & $\begin{array}{l}0.31 \\
0.32\end{array}$ & $\begin{array}{l}3.57 \\
-\end{array}$ & $\begin{array}{l}1.52 \\
-\end{array}$ & & & $\begin{array}{l}101.82 \\
100.00\end{array}$ & & Chr. Winther & O. B. Bøggild & r. v. R. N. & $17^{*}$ \\
\hline $\begin{array}{l}0.88 \\
1.24\end{array}$ & $\mathrm{tr}$. & $\begin{array}{c}10.39 \\
-\end{array}$ & 8.54 & $\begin{array}{l}0.39 \\
-\end{array}$ & & $\begin{array}{l}100.27 \\
100.01\end{array}$ & & Rolf Norin & Rolf Norin & r. v. R. N. & $18^{*}$ \\
\hline
\end{tabular}




\begin{tabular}{|c|c|c|c|c|c|c|c|c|c|c|c|}
\hline No. & Rock Name & Locality & Year & $\mathrm{SiO}_{2}$ & $\mathrm{TiO}_{2}$ & $\mathrm{Al}_{2} \mathrm{O}_{3}$ & $\mathrm{Fe}_{2} \mathrm{O}_{3}$ & $\mathrm{FeO}$ & $\mathrm{MnO}$ & $\mathrm{MgO}$ & $\mathrm{CaO}$ \\
\hline $19^{*}$ & Ash-layer -12 & Fur & 1940 & $\begin{array}{l}49.93 \\
53.55\end{array}$ & $\begin{array}{l}4.46 \\
4.77\end{array}$ & $\begin{array}{l}11.45 \\
12.23\end{array}$ & $\begin{array}{l}6.29 \\
6.72\end{array}$ & $\begin{array}{l}6.50 \\
6.95\end{array}$ & $\begin{array}{l}0.97 \\
1.04\end{array}$ & $\begin{array}{l}3.14 \\
3.36\end{array}$ & $\begin{array}{l}7.1 \\
7.0\end{array}$ \\
\hline $20 *$ & Ash-layer +19 & Ølst & 1940 & $\begin{array}{l}48.37 \\
70.23\end{array}$ & $\begin{array}{l}1.18 \\
1.72\end{array}$ & $\begin{array}{l}10.18 \\
14.77\end{array}$ & $\begin{array}{r}13.34 \\
2.37\end{array}$ & $\begin{array}{l}0.39 \\
0.58\end{array}$ & $\begin{array}{l}0.05 \\
0.07\end{array}$ & $\begin{array}{l}4.00 \\
5.81\end{array}$ & $\begin{array}{l}1.9 \\
2.0\end{array}$ \\
\hline $21^{*}$ & Ash-layer -12 & Ølst & 1940 & $\begin{array}{l}46.33 \\
55.43\end{array}$ & $\begin{array}{l}4.00 \\
4.79\end{array}$ & $\begin{array}{l}11.66 \\
13.95\end{array}$ & $\begin{array}{r}9.10 \\
10.89\end{array}$ & $\begin{array}{l}2.59 \\
3.10\end{array}$ & $\begin{array}{l}0.58 \\
0.69\end{array}$ & $\begin{array}{l}2.59 \\
3.10\end{array}$ & $\begin{array}{l}3.8 \\
4.1\end{array}$ \\
\hline $22 *$ & Ash-layer +101 & Hanklit & 1940 & $\begin{array}{l}29.57 \\
48.19\end{array}$ & $\begin{array}{l}3.00 \\
4.89\end{array}$ & $\begin{array}{r}8.34 \\
13.59\end{array}$ & $\begin{array}{l}2.89 \\
4.71\end{array}$ & $\begin{array}{l}6.12 \\
7.24\end{array}$ & $\begin{array}{l}0.30 \\
0.49\end{array}$ & $\begin{array}{l}3.16 \\
3.83\end{array}$ & $\begin{array}{l}24.6 \\
11.2\end{array}$ \\
\hline $23^{*}$ & Ash-layer +101 & Fur & 1940 & $\begin{array}{l}26.16 \\
46,57\end{array}$ & $\begin{array}{l}3.38 \\
6.02\end{array}$ & $\begin{array}{r}7.13 \\
12.69\end{array}$ & $\begin{array}{l}2.44 \\
4.34\end{array}$ & $\begin{array}{l}9.11 \\
7.90\end{array}$ & $\begin{array}{l}0.42 \\
0.76\end{array}$ & $\begin{array}{l}4.03 \\
4.18\end{array}$ & $\begin{array}{l}24.0 \\
11.5\end{array}$ \\
\hline $24 *$ & Ash-layer +101 & Helgenæs, Djursland & 1940 & $\begin{array}{l}26.01 \\
41.15\end{array}$ & $\begin{array}{l}3.28 \\
5.19\end{array}$ & $\begin{array}{l}11.34 \\
17.94\end{array}$ & $\begin{array}{l}4.18 \\
6.60\end{array}$ & $\begin{array}{l}4.45 \\
7.04\end{array}$ & $\begin{array}{l}2.25 \\
3.56\end{array}$ & $\begin{array}{l}2.33 \\
3.69\end{array}$ & $\begin{array}{r}23.0 \\
8.3\end{array}$ \\
\hline
\end{tabular}

The Faeroes.

\begin{tabular}{|c|c|c|c|c|c|c|c|c|c|c|c|}
\hline No. & Rock Name & Locality & Year & $\mathrm{SiO}_{2}$ & $\mathrm{TiO}_{2}$ & $\mathrm{Al}_{2} \mathrm{O}_{3}$ & $\mathrm{Fe}_{2} \mathrm{O}_{3}$ & $\mathrm{FeO}$ & $\mathrm{MnO}$ & $\mathrm{MgO}$ & $\mathrm{CaC}$ \\
\hline 25 & "Trapp anhydré" & Strømø & 1841 & 53.20 & & 13.26 & 2.23 & 15.83 & 0.50 & 9.16 & 5.1 \\
\hline 26 & Augite-andesite & Kolter & 1884 & 52.30 & & 18.22 & & 11.50 & & 7.24 & 10.2 \\
\hline 27 & Non-porphyritic basalt & Vaag Fjord, Suðerø & 1918 & 49.70 & 4.83 & 10.98 & 3.02 & 11.19 & 0.22 & 5.34 & 9.7 \\
\hline 28 & Trapp & $\begin{array}{l}\text { Height west of Torshavn, } \\
\text { Strømø }\end{array}$ & 1853 & 49.40 & & 14.42 & & 16.27 & & 5.86 & 10.3 \\
\hline $28 \mathrm{a}$ & Big-felspar basalt & Velbestad, Strømø & 1936 & 48.10 & 2.50 & 16.01 & 3.47 & 7.16 & 0.15 & 6.00 & 10.5 \\
\hline 29 & Olivine-dolerite & $\begin{array}{l}\text { Froðbiarkambur, } \\
\text { Tværaa, Suðerø }\end{array}$ & 1936 & 48.02 & 2.20 & 14.17 & 3.62 & 8.34 & 0.16 & 7.08 & 11.0 \\
\hline 30 & $\begin{array}{l}\text { Basalt with porphyritic } \\
\text { felspars }\end{array}$ & Torshavn, Strømø & 1922 & 47.58 & 2.72 & 17.12 & 3.03 & 8.73 & 0.14 & 5.18 & 10.1 \\
\hline 31 & $\begin{array}{l}\text { Zeolitic, amygdaloidal } \\
\text { rock }\end{array}$ & $\begin{array}{l}\text { Height west of Torshavn, } \\
\text { Strømø }\end{array}$ & 1853 & 47.46 & & 12.72 & & 14.49 & & 5.63 & 8. \\
\hline 32 & "Trapp hydraté" & Strømø & 1841 & 46.80 & & 14.40 & 2.30 & 9.90 & 2.80 & 9.53 & 10.1 \\
\hline 33 & $\begin{array}{l}\text { Zeolitic, amygdaloidal } \\
\text { rock (altered) }\end{array}$ & $\begin{array}{l}\text { Height west of Torshavn, } \\
\text { Strømø }\end{array}$ & 1853 & 46.65 & & 14.66 & & 14.61 & & 6.88 & 8.8 \\
\hline
\end{tabular}




\begin{tabular}{l|c|c|c||c|c|c|c|c|c|c|c}
\hline \hline No. & \multicolumn{1}{|c|}{ Rock Name } & Locality & Year & $\mathrm{SiO}_{2}$ & $\mathrm{TiO}_{2}$ & $\mathrm{Al}_{2} \mathrm{O}_{3}$ & $\mathrm{Fe}_{2} \mathrm{O}_{3}$ & $\mathrm{FeO}$ & $\mathrm{MnO}$ & $\mathrm{MgO}$ & $\mathrm{CaC}$ \\
\hline 34 & $\begin{array}{l}\text { Basalt with porphyritic } \\
\text { felspars } \\
\text { Olivine-basalt }\end{array}$ & Höyvik, Strømø & 1928 & 46.40 & 3.05 & 16.30 & 3.60 & 7.17 & 0.23 & 6.00 & 11.0 \\
35 & Trangisvaag, Suðerø & 1928 & 45.40 & 3.20 & 14.54 & 1.96 & 10.45 & 0.25 & 6.25 & 11.7 \\
36 & Ultramafic olivine-basalt & $\begin{array}{l}\text { Southeast of church, } \\
\text { Ejde, Østerø } \\
\text { Height west of Torshavn, } \\
\text { Strømø }\end{array}$ & 1936 & 44.40 & 0.75 & 12.25 & 1.50 & 8.92 & 0.18 & 17.66 & 10.0 \\
\hline $37^{*}$ & Altered palagonite-tuff & 40.69 & & 15.31 & & 13.44 & & 6.31 & 3.3 \\
\hline
\end{tabular}

\section{Greenland.}

\begin{tabular}{|c|c|c|c|c|c|c|c|c|c|c|c|c|}
\hline No. & Rock Name & Locality & District & Year & $\mathrm{SiO}_{2}$ & $\mathrm{TiO}_{2}$ & $\mathrm{Al}_{2} \mathrm{O}_{3}$ & $\mathrm{Fe}_{2} \mathrm{O}_{3}$ & $\mathrm{FeO}$ & $\mathrm{MnO}$ & $\mathrm{MgO}$ & $\mathrm{CaC}$ \\
\hline 38 & Sanidine-rhyolite & Cape Franklin & NE & 1935 & 87.96 & 0.10 & 5.35 & 1.09 & 0.45 & tr. & 0.44 & \\
\hline 39 & Sanidine-rhyolite & Cape Franklin & NE & 1935 & 79.24 & 0.15 & 9.74 & 1.85 & 0.35 & 0.04 & 0.15 & 0.32 \\
\hline 40 & Gneiss, psammitic & $\begin{array}{l}\text { Hurry Inlet side of } \\
\text { Liverpool Land }\end{array}$ & NE & 1935 & 78.63 & 0.60 & 8.91 & 2.12 & 1.03 & 0.04 & 0.62 & 2.1 \\
\hline 41 & Alkali-rhyolite & $\begin{array}{l}\text { Cape Graah, } \\
\text { Ymer Island. }\end{array}$ & NE & 1940 & 77.02 & 0.24 & 11.24 & 0.23 & 0.73 & 0.01 & 0.24 & \\
\hline 42 & Alkali-rhyolite & Musk Ox Fjord & NE & 1940 & 76.92 & 0.22 & 12.12 & 0.73 & 0.59 & 0.02 & 0.04 & 0.64 \\
\hline 43 & “Quartz-keratophyre“ & $\begin{array}{l}\text { Mt. Ramsay, } \\
\text { Gauss Peninsula }\end{array}$ & NE & 1932 & 76.51 & 0.08 & 11.59 & 0.99 & 0.56 & 0.02 & 0.25 & 0.48 \\
\hline 44 & Granophyre-granite & $\begin{array}{l}\text { Mt. Högbom, } \\
\text { Gauss Peninsula }\end{array}$ & NE & 1932 & 76.04 & 0.12 & 12.03 & 1.37 & 0.22 & 0.01 & 0.05 & \\
\hline 45 & Granite & Umanak Island & NW & 1904 & 76.03 & 0.28 & 12.02 & 0.69 & 0.68 & & 0.18 & 1.61 \\
\hline 46 & $\begin{array}{l}\text { Acid orthoclase-bearing } \\
\text { porphyry }\end{array}$ & $\begin{array}{l}\text { Cape Fletcher, } \\
\text { Canning Land }\end{array}$ & NE & 1907 & 75.14 & 0.16 & 12.50 & 1.20 & 0.87 & tr. & 0.43 & 0.83 \\
\hline 47 & Acid granophyre & $\begin{array}{l}\text { Tinden, } \\
\quad \text { Kangerdlugssuaq }\end{array}$ & SE & 1939 & 75.03 & 0.31 & 13.17 & 1.56 & 0.58 & 0.01 & 0.15 & 0.1 \\
\hline 48 & Quartz-rhyolite & Cape Franklin & NE & $\begin{array}{c}1932 \\
(1935)\end{array}$ & 74.97 & 0.11 & 12.90 & 1.21 & 0.29 & 0.01 & 0.11 & 0. \\
\hline 49 & Alkali-granite & Ivigtut Peninsula & SW & & 74.80 & $\operatorname{tr}$ & 12.37 & 2.06 & 1.24 & tr. & 0.16 & \\
\hline 50 & Porphyry granite & $\begin{array}{l}\text { Cape Oswald, } \\
\text { Ella Island }\end{array}$ & $\mathrm{NE}$ & 1932 & 74.79 & 0.16 & 12.09 & 0.93 & 1.13 & 0.02 & 0.54 & \\
\hline
\end{tabular}




\begin{tabular}{|c|c|c|c|c|c|c|c|c|c|c|c|c|}
\hline $\mathrm{Na}_{2} \mathrm{O}$ & $\mathrm{K}_{2} \mathrm{O}$ & $\mathrm{P}_{2} \mathrm{O}_{5}$ & $\mathrm{H}_{2} \mathrm{O}+$ & $\mathrm{H}_{2} \mathrm{O}^{-}$ & $\mathrm{CO}_{2}$ & Inclusive & Sum & Sp.gr. & Analyst & Published by & Notes & No. \\
\hline 2.14 & 0.29 & 0.23 & 1.10 & 2.40 & nil & $\mathrm{S}: \mathrm{tr}$ & 99.95 & & W.H.Herdsmann & I. B. Simpson & & 34 \\
\hline 2.08 & 0.49 & 0.24 & 1.50 & 2.00 & nil & $\mathrm{S}: \mathrm{tr}$ & 100.08 & & W.H.Herdsmann & I. B. Simpson & & 35 \\
\hline 0.95 & 0.25 & 0.04 & 1.60 & 1.60 & nil & CuO : nil & 100.10 & & W.H.Herdsmann & $\begin{array}{l}\text { Walker \& } \\
\text { Davidson }\end{array}$ & & 36 \\
\hline 0.60 & 0.74 & & 20.00 & & & & 100.48 & & A. Streng & A. Streng & & $37^{*}$ \\
\hline
\end{tabular}

Greenland.

\begin{tabular}{|c|c|c|c|c|c|c|c|c|c|c|c|c|}
\hline $\mathrm{Na}_{2} \mathrm{O}$ & $\mathrm{K}_{2} \mathrm{O}$ & $\mathrm{P}_{2} \mathrm{O}_{5}$ & $\mathrm{H}_{2} \mathrm{O}+$ & $\mathrm{H}_{2} \mathrm{O}^{-}$ & $\mathrm{CO}_{2}$ & Inclusive & Sum & Sp.gr. & Analyst & Published by & Notes & No. \\
\hline 0.52 & 1.52 & tr. & 1.28 & 0.35 & & & 100.30 & 2.638 & N. Sahlbom & $\begin{array}{l}\text { H. Backlund \& } \\
\text { D. Malmquist }\end{array}$ & & 38 \\
\hline 2.35 & 4.64 & tr. & 0.90 & 0.20 & & & 99.93 & 2.520 & N. Sahlbom & $\begin{array}{l}\text { H. Backlund \& } \\
\text { D. Malmquist }\end{array}$ & & 39 \\
\hline 1.33 & 3.83 & 0.05 & 0.46 & 0.10 & & $\mathrm{ZrO}_{2}: 0.05$ & 99.89 & & N. Sahlbom & E. H. Kranck & & 40 \\
\hline 2.22 & 6.42 & 0.14 & 0.48 & 0.07 & & & 99.92 & & N. Sahlbom & A. Rittmann & & 41 \\
\hline 2.92 & 4.93 & tr. & 0.76 & 0.18 & & & 100.07 & & N. Sahlbom & A. Rittmann & & 42 \\
\hline 1.71 & 6.87 & tr. & 0.49 & 0.26 & 0.09 & & $\begin{array}{c}99.90 \\
(99.89)\end{array}$ & & N. Sahlbom & H. G. Backlund & & 43 \\
\hline 2.66 & 6.54 & 0.30 & 0.60 & 0.17 & & & $\begin{array}{c}100.63 \\
(100.53)\end{array}$ & & N. Sahlbom & H. G. Backlund & & 44 \\
\hline 2.97 & 5.72 & & 0.20 & & & & 100.38 & & W. C. Phalen & W. C. Phalen & & 45 \\
\hline 3.00 & 3.50 & & & & & $\begin{array}{l}\text { Loss on } \\
\text { ignition : } 2.55\end{array}$ & 100.18 & & N. Sahlbom & O. Nordenskjöld & & 46 \\
\hline 4.24 & 3.85 & 0.02 & 0.28 & 0.13 & & & 100.02 & 2.54 & W. A. Deer & $\begin{array}{l}\text { L. R. Wager \& } \\
\text { W. A. Deer }\end{array}$ & & 47 \\
\hline 0.16 & 7.66 & tr. & 1.60 & 0.29 & & $\mathrm{BaO}$ : nil & 99.99 & 2.556 & N. Sahlbom & $\begin{array}{l}\text { (H. G. Backlund) } \\
\text { H. Backlund \& } \\
\text { D. Malmquist }\end{array}$ & & 48 \\
\hline 4.08 & 3.64 & tr. & 0.93 & 0.15 & tr. & $\mathrm{F}: 0.38$ & $\begin{array}{r}100.06 \\
-0.17 \\
\end{array}$ & & W.H.Herdsmann & (R. Bøgvad) & $\begin{array}{l}\text { Unpublis- } \\
\text { hed (sml. }\end{array}$ & 49 \\
\hline 2.99 & 5.59 & 0.10 & 0.81 & 0.15 & & & $\begin{array}{c}99.89 \\
99.92 \\
(99.87)\end{array}$ & & N. Sahlbom & H. G. Backlund & $\begin{array}{l}\text { no. } 105 \& \\
\text { no. } 112)\end{array}$ & 50 \\
\hline
\end{tabular}




\begin{tabular}{|c|c|c|c|c|c|c|c|c|c|c|c|c|}
\hline No. & Rock Name & Locality & District & Year & $\mathrm{SiO}_{2}$ & $\mathrm{TiO}_{2}$ & $\mathrm{Al}_{2} \mathrm{O}_{3}$ & $\mathrm{Fe}_{2} \mathrm{O}_{3}$ & $\mathrm{FeO}$ & $\mathrm{MnO}$ & $\mathrm{MgO}$ & $\mathrm{CaO}$ \\
\hline 51 & Sanidine-rhyolite & Cape Franklin & $\mathrm{NE}$ & 1935 & 73.95 & 0.13 & 12.44 & 1.68 & 0.41 & 0.02 & 0.05 & 1.38 \\
\hline 52 & Quartz-porphyry & Ilimausak & SW & 1911 & 73.68 & 0.57 & 11.05 & 3.93 & 1.45 & tr. & nil & 0.48 \\
\hline 53 & Rhyolite, spherulitic & Cape Franklin & NE & 1935 & 73.36 & 0.15 & 11.99 & 2.76 & 0.83 & 0.02 & 0.24 & 1.78 \\
\hline 54 & $\begin{array}{l}\text { Porphyry (with pheno- } \\
\text { crystic quartz) }\end{array}$ & $\begin{array}{l}\text { Mt. Nordenskjöld, } \\
\text { Nathorst Fjord }\end{array}$ & $\mathrm{NE}$ & $\begin{array}{c}(1936) \\
1937\end{array}$ & 73.12 & 0.32 & 13.64 & 1.30 & 0.90 & 0.06 & 0.40 & 0.86 \\
\hline 55 & Granite & $\begin{array}{l}\text { Mt. la Cour, } \\
\text { Gauss Peninsula }\end{array}$ & $\mathrm{NE}$ & 1932 & 73.11 & tr. & 14.01 & 0.09 & 0.21 & 0.01 & 0.29 & 1.36 \\
\hline 56 & Rhyolite, spherulite & Cape Franklin & $\mathrm{NE}$ & $\begin{array}{c}(1932) \\
1935\end{array}$ & 72.35 & 0.14 & 14.03 & 1.54 & 0.48 & 0.01 & 0.33 & 0.86 \\
\hline 57 & Two-mica-granite & Kempe Fjord & $\mathrm{NE}$ & 1932 & 72.29 & 0.24 & 15.09 & 0.41 & 1.01 & tr. & 0.34 & 0.74 \\
\hline 58 & Two-mica-granite & Forsblad Fjord & $\mathrm{NE}$ & 1932 & 72.17 & 0.33 & 14.03 & 0.73 & 1.31 & 0.01 & 0.61 & 0.72 \\
\hline 59 & Two-mica-granite & Clavering Island & $\mathrm{NE}$ & 1932 & 71.95 & 0.11 & 14.59 & 1.01 & 0.72 & 0.01 & 0.33 & 1.05 \\
\hline 60 & Two-mica-granite & Forsblad Fjord & $\mathrm{NE}$ & 1932 & 71.90 & 0.30 & 14.69 & 0.67 & 0.80 & 0.01 & 0.33 & 1.04 \\
\hline 61 & Soda-granite & $\begin{array}{l}\text { Iviangussat, } \\
\text { Kangerdluarssuk }\end{array}$ & SW & 1911 & 71.24 & 0.68 & 13.78 & 1.30 & 2.83 & 0.15 & tr. & 0.38 \\
\hline 62 & Granite, fine-grained & Liverpool Land & NE & 1935 & 70.83 & 0.18 & 14.63 & 2.28 & 1.18 & 0.02 & 0.51 & 1.80 \\
\hline 63 & $\begin{array}{l}\text { Spherulite (from sphe- } \\
\text { rulitic rhyolite) }\end{array}$ & Cape Franklin & $\mathrm{NE}$ & 1935 & 70.80 & 0.08 & 9.42 & 3.45 & 3.01 & 0.40 & 0.85 & 1.47 \\
\hline 64 & $\begin{array}{l}\text { Chlorite-oligoclase- } \\
\text { microcline gneiss }\end{array}$ & Musk Ox Fjord & $\mathrm{NE}$ & 1931 & 70.75 & 0.28 & 13.58 & 0.70 & 1.88 & nil & 0.72 & 2.10 \\
\hline 65 & $\begin{array}{l}\text { Granite, coarse } \\
\text { porphyric }\end{array}$ & Liverpool Land & $\mathrm{NE}$ & 1935 & 70.73 & 0.36 & 15.36 & 0.81 & 1.17 & 0.02 & 0.88 & 2.40 \\
\hline 66 & Arfvedsonite-granite & Ilimausak & SW & 1911 & 70.59 & 0.44 & 12.38 & 1.61 & 3.33 & 0.08 & nil & 0.93 \\
\hline 67 & Biotite-granite & $\begin{array}{l}\text { Cape Wardlaw, } \\
\text { Canning Land }\end{array}$ & $\mathrm{NE}$ & $\begin{array}{l}(1936) \\
1937\end{array}$ & 69.78 & 0.50 & 14.11 & 0.36 & 2.36 & 0.03 & 1.35 & 1.60 \\
\hline 68 & Granite & $\begin{array}{l}\text { Ättestupan, Franz } \\
\text { Joseph Fjord }\end{array}$ & $\mathrm{NE}$ & 1932 & 69.66 & 0.31 & 14.42 & 0.98 & 1.30 & 0.02 & 1.15 & 1.40 \\
\hline 69 & Gneiss & Umanak Island & NW & 1904 & 69.07 & 0.78 & 14.09 & 1.49 & 2.37 & tr. & 0.98 & 3.14 \\
\hline 70 & Gneiss, grey & $\begin{array}{l}\text { Siusasigsak, north } \\
\text { of Jakobshavn }\end{array}$ & NW & 1905 & 69.06 & 0.74 & 15.26 & 0.68 & 2.53 & & 0.34 & 2.83 \\
\hline 71 & Microcline-granite & $\begin{array}{l}\text { Store Fjord, } \\
\text { Liverpool Land }\end{array}$ & NE & 1935 & 68.80 & 0.38 & 15.98 & 1.63 & 1.47 & 0.04 & 1.02 & 2.52 \\
\hline
\end{tabular}




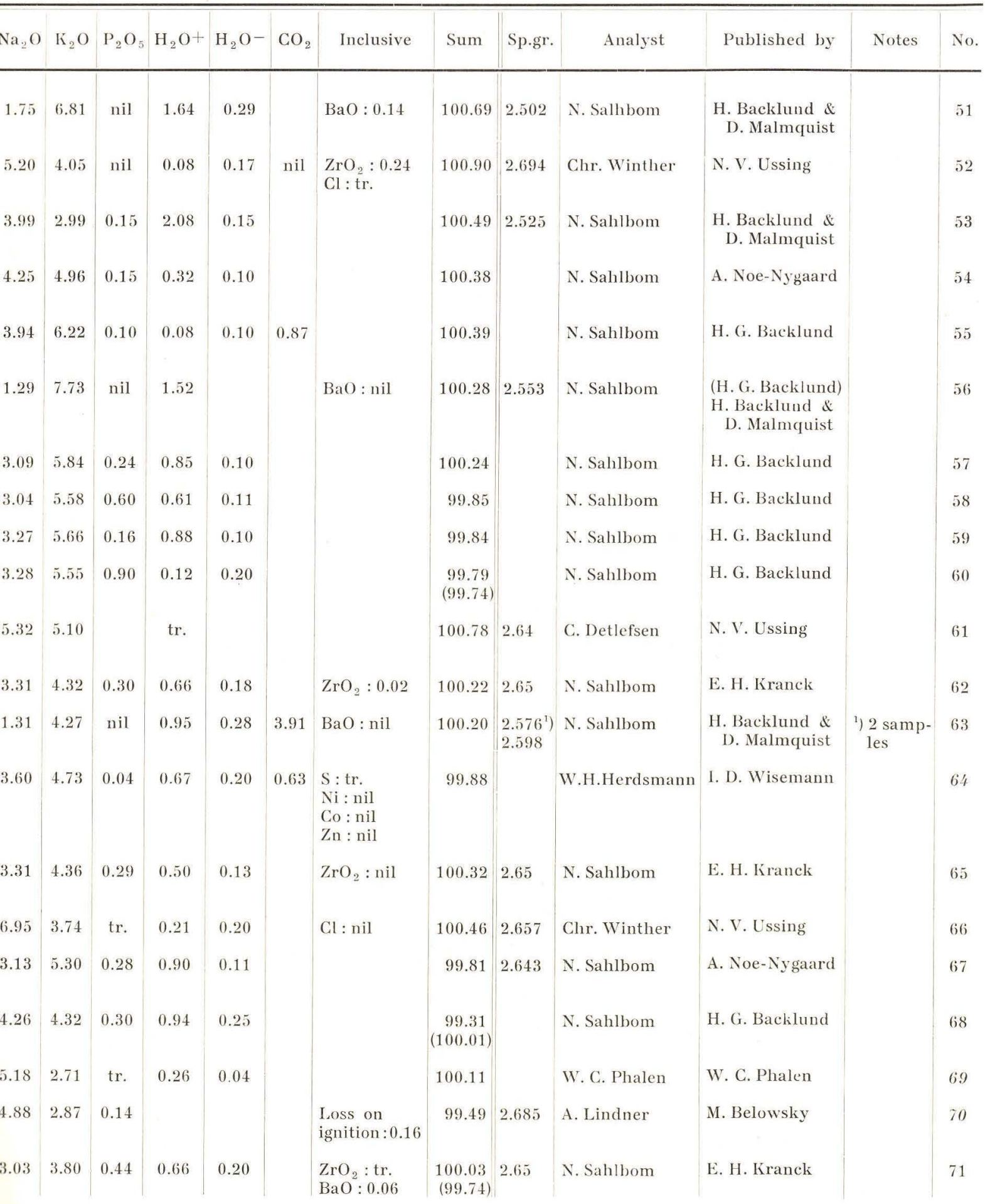




\begin{tabular}{|c|c|c|c|c|c|c|c|c|c|c|c|c|}
\hline No. & Rock Name & Locality & District & Year & $\mathrm{SiO}_{2}$ & $\mathrm{TiO}_{2}$ & $\mathrm{Al}_{2} \mathrm{O}_{3}$ & $\mathrm{Fe}_{2} \mathrm{O}_{3}$ & $\mathrm{FeO}$ & $\mathrm{MnO}$ & $\mathrm{MgO}$ & $\mathrm{Cal}$ \\
\hline 72 & $\begin{array}{l}\text { Rhyolitic eruption- } \\
\text { breccia }\end{array}$ & Cape Franklin & $\mathrm{NE}$ & 1935 & 68.74 & 0.90 & 10.65 & 2.84 & 5.94 & 0.10 & 2.52 & 0.7 \\
\hline 73 & Gneiss, grey & $\begin{array}{l}\text { Cape Weber, } \\
\text { Andrée Land }\end{array}$ & $\mathrm{NE}$ & 1932 & 68.59 & 0.38 & 14.43 & 1.06 & 2.09 & 0.02 & 1.23 & 2.3 \\
\hline 74 & Gneiss, grey & $\begin{array}{l}\text { Mellemø, } \\
\text { Kangerdlugssuaq }\end{array}$ & SE & 1939 & 68.17 & 0.63 & 16.13 & 0.58 & 2.09 & 0.05 & 1.82 & $2.0^{\circ}$ \\
\hline 75 & $\begin{array}{l}\text { Red porphyry (with } \\
\text { phenocrystic quartz) }\end{array}$ & $\begin{array}{l}\text { Tvekegledal, Wege- } \\
\text { ner Peninsula }\end{array}$ & $\mathrm{NE}$ & $\begin{array}{c}(1936) \\
1937\end{array}$ & 67.93 & 0.70 & 13.92 & 1.61 & 1.50 & 0.02 & 1.45 & 3.1 : \\
\hline 76 & Quartz-monzonite & Nûgssuaq Peninsula & NW & 1904 & 67.27 & 1.70 & 13.67 & 1.83 & 2.49 & 0.19 & 1.72 & 1.9 \\
\hline 77 & $\begin{array}{l}\text { Granite (biotite- } \\
\text { muscovite-granite) }\end{array}$ & $\begin{array}{l}\text { Øve Rypegletcher, } \\
\text { Franz Joseph Fjord }\end{array}$ & NE & 1930 & 66.57 & 0.78 & 11.42 & 3.30 & 3.68 & 0.10 & 1.38 & 3.1 \\
\hline 78 & $\begin{array}{l}\text { Grey porphyry (with } \\
\text { phenocrystic quartz) }\end{array}$ & $\begin{array}{l}\text { Hesteskofjeld, } \\
\text { Canning Land }\end{array}$ & NE & $\begin{array}{c}(1936) \\
1937\end{array}$ & 66.42 & 0.76 & 15.31 & 1.03 & 2.74 & 0.02 & 1.46 & 2.0 \\
\hline 79 & $\begin{array}{l}\text { Greyish-green porphyry } \\
\text { (without phenocrystic } \\
\text { quartz) }\end{array}$ & $\begin{array}{l}\text { Mt. Kollen, } \\
\text { Canning Land }\end{array}$ & NE & $\begin{array}{c}(1936) \\
1937\end{array}$ & 66.41 & 0.80 & 15.38 & 0.74 & 2.34 & 0.05 & 2.23 & 1.98 \\
\hline 80 & Porphyric granite & $\begin{array}{l}\text { Porfyrfjeld, } \\
\text { Canning Land }\end{array}$ & $\mathrm{NE}$ & $\begin{array}{c}(1936) \\
1937\end{array}$ & 65.57 & 0.56 & 14.57 & 0.63 & 2.16 & 0.04 & 1.17 & 3.96 \\
\hline 81 & $\begin{array}{l}\text { Riebeckite-trachyte } \\
\text { (spherulitic) }\end{array}$ & $\begin{array}{l}\text { Cape Parry, Traill } \\
\text { Island }\end{array}$ & NE & 1932 & 64.82 & 0.33 & 14.29 & 2.04 & 4.27 & tr. & 1.00 & 1.5 \\
\hline 82 & Biotite-dacite & $\begin{array}{l}\text { Segelsällskapets } \\
\text { Fjord }\end{array}$ & $\mathrm{NE}$ & 1940 & 64.36 & 0.88 & 15.51 & 0.29 & 4.14 & 0.04 & 2.25 & 3.51 \\
\hline 83 & $\begin{array}{l}\text { Porphyry, red (with } \\
\text { phenocrystic quartz) }\end{array}$ & $\begin{array}{l}\text { Porfyrfjeld, } \\
\quad \text { Canning Land }\end{array}$ & $\mathrm{NE}$ & $\begin{array}{c}(1936) \\
1937\end{array}$ & 63.90 & 0.72 & 14.57 & 1.11 & 2.86 & 0.03 & 1.11 & 3.46 \\
\hline 84 & Gneiss, granulitic & $\begin{array}{l}\text { Cape Hope, } \\
\text { Liverpool Land }\end{array}$ & NE & 1935 & 63.86 & 1.60 & 13.45 & 3.28 & 5.33 & 0.10 & 4.16 & 2.04 \\
\hline 85 & Anorthoclase-trachyte & $\begin{array}{l}\text { Arfertuarssuk Fjord } \\
\text { Svartenhuk } \\
\text { Peninsula }\end{array}$ & NW & 1931 & 63.32 & 0.75 & 16.80 & 3.89 & 1.25 & 0.15 & 0.85 & 2.07 \\
\hline 86 & Astochite-gneiss & Qarajaq Glacier & NW & 1905 & 63.26 & 0.20 & 14.84 & 2.39 & 1.54 & tr. & 4.22 & 1.61 \\
\hline 87 & Syenite & $\begin{array}{l}\text { Antaretic Harbour, } \\
\text { King Oscar Fjord }\end{array}$ & $\mathrm{NE}$ & 1940 & 62.10 & 0.92 & 17.76 & 1.75 & 2.14 & 0.06 & 0.98 & 2.34 \\
\hline 88 & $\begin{array}{l}\text { Nepheline-sodalite- } \\
\text { syenite }\end{array}$ & Kangerdlugssuaq & SE & 1934 & 61.70 & 0.80 & 18.35 & 1.63 & 1.62 & 0.32 & 0.61 & 0.95 \\
\hline
\end{tabular}




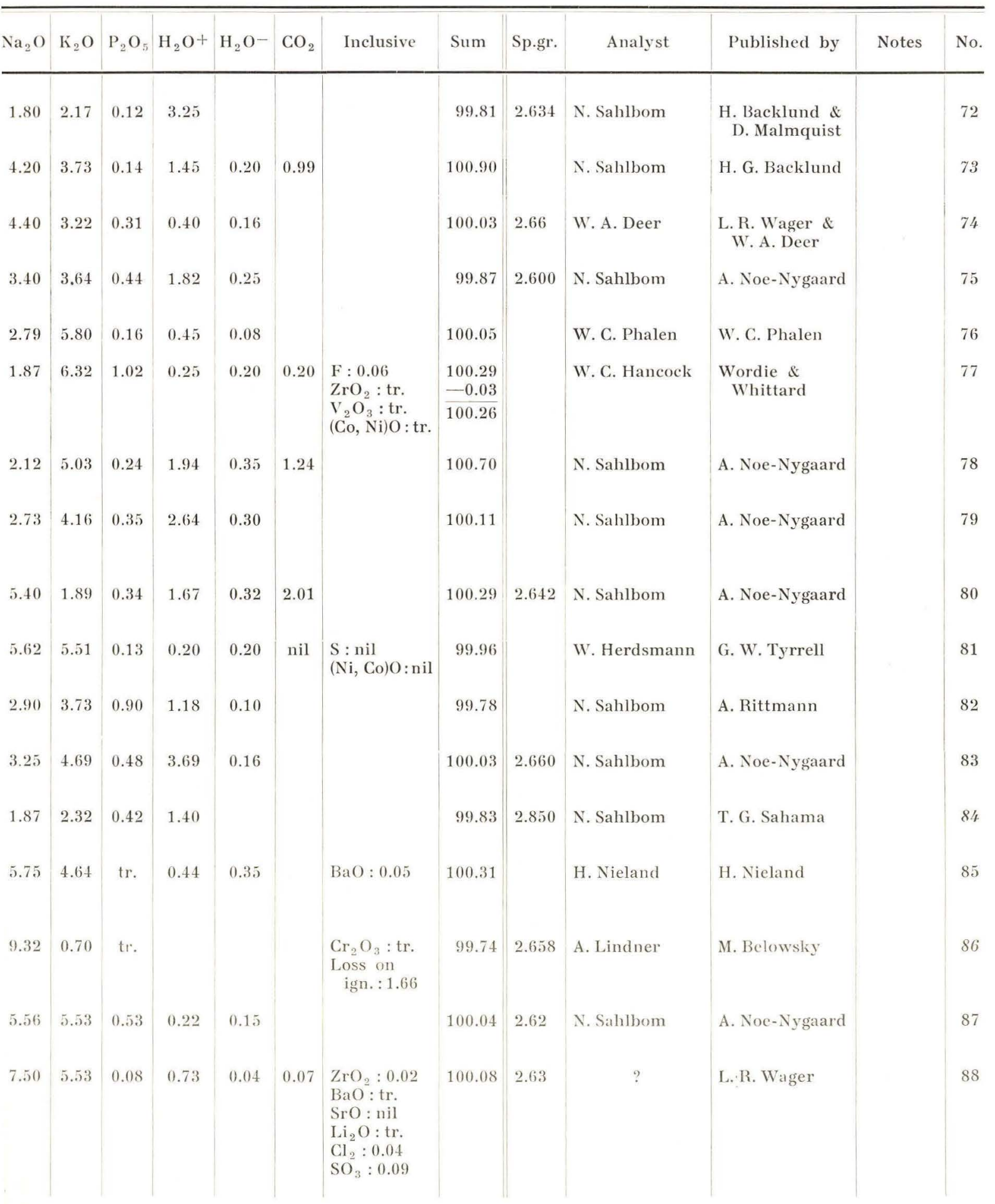




\begin{tabular}{|c|c|c|c|c|c|c|c|c|c|c|c|c|}
\hline No. & Rock Name & Locality & District & Year & $\mathrm{SiO}_{2}$ & $\mathrm{TiO}_{2}$ & $\mathrm{Al}_{2} \mathrm{O}_{3}$ & $\mathrm{Fe}_{2} \mathrm{O}_{3}$ & $\mathrm{FeO}$ & $\mathrm{MnO}$ & $\mathrm{MgO}$ & $\mathrm{CaO}$ \\
\hline 89 & $\begin{array}{l}\text { Porphyry, reddish- } \\
\text { brown (without phe- } \\
\text { nocrystic quartz) }\end{array}$ & $\begin{array}{l}\text { Porfyrfjeld, } \\
\text { Canning Land }\end{array}$ & NE & $\begin{array}{c}(1936) \\
1937\end{array}$ & 61.66 & 0.54 & 15.07 & 2.10 & 3.23 & 0.06 & 1.92 & 3.82 \\
\hline 90 & $\begin{array}{l}\text { Porphyry, greyish- } \\
\text { green (withoutpheno- } \\
\text { crystic quartz) }\end{array}$ & Cape Fletcher & NE & $\begin{array}{c}(1936) \\
1937\end{array}$ & 61.53 & 1.01 & 15.07 & 2.23 & 2.89 & 0.08 & 3.36 & 3.59 \\
\hline 91 & $\begin{array}{l}\text { Aplitic mass from } \\
\text { Kaersutite-pegmatite }\end{array}$ & Osterfjeld, Qaersut & NW & 1928 & 59.77 & 0.81 & 18.14 & 1.04 & 1.90 & 0.05 & 1.76 & 1.67 \\
\hline 92 & Iron-bearing rock & Ivigsarkut & $\mathrm{NW}$ & 1901 & 59.03 & 1.19 & 13.16 & nil. & 7.02 & & 5.50 & 6.75 \\
\hline 93 & $\begin{array}{l}\text { Porphyry, reddish- } \\
\text { brown (without phe- } \\
\text { nocrystic quartz) }\end{array}$ & Cape Fletcher & NE & $\begin{array}{c}(1936) \\
1937\end{array}$ & 58.98 & 1.20 & 14.09 & 4.76 & 1.96 & 0.10 & 3.98 & 4.40 \\
\hline 94 & Iron-bearing rock & Jernpynten, Disko & NW & 1901 & 58.91 & 1.23 & 13.43 & nil. & 7.15 & & 5.63 & 6.68 \\
\hline 95 & $\begin{array}{l}\text { Hedenbergite-grano- } \\
\text { phyre }\end{array}$ & $\begin{array}{l}\text { Brødretoppen, } \\
\text { Kangerdlugssuaq }\end{array}$ & $\mathrm{SE}$ & 1939 & 58.81 & 1.26 & 12.02 & 5.77 & 9.38 & 0.21 & 0.72 & 5.03 \\
\hline 96 & Granodiorite & $\begin{array}{l}\text { Cape Smith, } \\
\text { Liverpool Land }\end{array}$ & NE & 1935 & 58.45 & 1.48 & 17.93 & 3.83 & 1.39 & 0.04 & 2.12 & 5.98 \\
\hline 97 & Nordmarkite & Narsak & SW & 1911 & 58.17 & 2.09 & 16.07 & 1.30 & 5.04 & 0.07 & 1.20 & 3.42 \\
\hline 98 & Pulaskite & $\begin{array}{l}\text { N. Siorarsuit, } \\
\text { Tunugdliarfik }\end{array}$ & SW & 1911 & 57.88 & 1.23 & 14.80 & 5.86 & 3.71 & 0.15 & nil. & 2.71 \\
\hline 99 & Granodiorite & Raffles Island & NE & 1935 & 57.79 & 1.30 & 16.30 & 2.87 & 3.41 & 0.08 & 3.93 & 6.97 \\
\hline 100 & Acid quartz-gabbro & $\begin{array}{l}\text { Osttoppen, } \\
\text { Kangerdlugssuaq }\end{array}$ & $\mathrm{SE}$ & 1939 & 57.00 & 1.78 & 13.02 & 2.15 & 9.25 & 0.12 & 2.64 & 5.75 \\
\hline 101 & Hedrumite & $\begin{array}{l}\text { Akuliarusek, } \\
\text { Igaliko Fjord }\end{array}$ & SW & 1911 & 56.90 & 1.09 & 16.34 & 3.61 & 5.72 & $\operatorname{tr}$ & 0.22 & 2.21 \\
\hline 102 & Arfvedsonite-lujavrite & $\begin{array}{l}\text { Nunasarnak, } \\
\text { Tunugdliarfik }\end{array}$ & $\mathrm{SW}$ & 1911 & 56.64 & 0.30 & 16.10 & 4.90 & 6.86 & 0.57 & nil. & 0.39 \\
\hline 103 & Foyaite & $\begin{array}{l}\text { Naujakasik, } \\
\text { Tunugdliarfik }\end{array}$ & $\mathrm{SW}$ & 1911 & 56.31 & $\left.2.82^{1}\right)$ & 20.11 & 3.93 & 1.45 & 0.60 & 0.36 & 0.62 \\
\hline 104 & Augite-syenite & $\begin{array}{l}\text { Nunasarnausak, } \\
\text { Kangerdluarsuk }\end{array}$ & SW & 1911 & 55.79 & 1.81 & 15.76 & 1.60 & 7.56 & 0.14 & 0.41 & 3.70 \\
\hline 105 & cf. Leuko-syenite & Ivigtut Peninsula & $\mathrm{SW}$ & & 55.76 & nil. & 21.52 & 3.20 & 2.65 & 0.44 & 0.07 & 1.22 \\
\hline
\end{tabular}




\begin{tabular}{|c|c|c|c|c|c|c|c|c|c|c|c|c|}
\hline $\mathrm{Na}_{2} \mathrm{O}$ & $\mathrm{K}_{2} \mathrm{O}$ & $\mathrm{P}_{2} \mathrm{O}_{5}$ & $\mathrm{H}_{2} \mathrm{O}+$ & $\mathrm{H}_{2} \mathrm{O}^{-}$ & $\mathrm{CO}_{2}$ & Inclusive & Sum & Sp.gr. & Analyst & Published by & Notes & No. \\
\hline 2.36 & 3.87 & 0.58 & 4.65 & 0.48 & & & 100.34 & 2.697 & N. Sahlbom & A. Noe-Nygaard & & 89 \\
\hline 3.62 & 4.67 & 0.50 & 1.86 & 0.25 & & & 100.66 & 2.638 & N. Sahlbom & A. Noe-Nygaard & & 90 \\
\hline 5.68 & 6.33 & 0.20 & 1.82 & 0.30 & 0.35 & & 99.82 & 2.505 & Schäffer & $\begin{array}{r}\text { F. K. Drescher \& } \\
\text { H.K.E.Krueger }\end{array}$ & & 91 \\
\hline 2.47 & 1.43 & 0.13 & 2.86 & & 0.25 & & 99.79 & & M. Dittrich & Th. Nicolau & & 92 \\
\hline 2.95 & 3.68 & 0.48 & 3.22 & 0.30 & & & 100.10 & 2.683 & N. Sahlbom & A. Noe-Nygaard & & 93 \\
\hline 2.91 & 1.31 & 0.15 & 1.48 & & 1.35 & & 100.23 & & M. Dittrich & Th. Nicolau & & 94 \\
\hline 3.91 & 2.39 & 0.71 & 0.21 & 0.19 & & & 100.61 & 2.82 & W. A. Deer & $\begin{array}{l}\text { L. R. Wager \& } \\
\text { W. A. Deer }\end{array}$ & & 95 \\
\hline 4.03 & 2.94 & & 0.88 & 0.10 & & $\begin{array}{l}\mathrm{ZrO}_{2}: \mathrm{tr} . \\
\mathrm{BaO}: 0.04 \\
\mathrm{~F}: \text { nil }\end{array}$ & $\begin{array}{c}99.21 \\
(99.71)\end{array}$ & 2.75 & N. Sahlbom & E. H. Kranck & & 96 \\
\hline 7.41 & 4.65 & 0.42 & 0.41 & 0.19 & nil & $\begin{array}{l}\mathrm{Cl}: \text { nil } \\
\mathrm{SO}_{3}: \text { nil }\end{array}$ & 100.44 & 2.74 & Chr. Winther & N. V. Ussing & & 97 \\
\hline 9.12 & 3.06 & tr. & 0.90 & 0.23 & & $\begin{array}{l}\mathrm{Cl}: \text { nil } \\
\mathrm{SO}_{3}: \text { nil } \\
\mathrm{ZrO}_{2}: \text { nil }\end{array}$ & 99.65 & 2.772 & Chr. Winther & N. V. Ussing & & 98 \\
\hline 2.71 & 2.98 & 0.67 & 0.62 & 0.13 & & $\begin{array}{l}\mathrm{BaO}: 0.12 \\
\mathrm{~S}: 0.09\end{array}$ & $\begin{array}{c}99.97 \\
(99.98)\end{array}$ & 2.75 & N. Sahlbom & E. H. Kranck & & 99 \\
\hline 4.11 & 0.99 & 2.11 & 0.97 & 0.41 & & & 100.30 & 3.02 & W. R. Deer & $\begin{array}{c}\text { L. A. Wager \& } \\
\text { W. A. Deer }\end{array}$ & & 100 \\
\hline 8.10 & 4.96 & 0.17 & 1.10 & 0.08 & & $\mathrm{Cl}: \mathrm{tr}$. & 100.50 & 2.783 & Chr. Winther & N. V. Ussing & & 101 \\
\hline 11.50 & 1.00 & nil. & 1.54 & 0.04 & & $\begin{array}{l}\mathrm{Cl}: \mathrm{tr} . \\
\mathrm{SO}_{3}: \text { nil } \\
\mathrm{Nb}_{2} \mathrm{O}_{5}: 0.45\end{array}$ & 100.29 & 2.79 & Chr. Christensen & N. V. Ussing & & 102 \\
\hline 8.76 & 4.65 & 0.13 & 1.13 & & & $\mathrm{Cl}: 0.15$ & $\begin{array}{r}101.02 \\
-0.03 \\
100.99\end{array}$ & 2.67 & C. Detlefsen & N. V. Ussing & $\begin{array}{l}\text { 1) Incl. } \\
\mathrm{ZrO}_{2}\end{array}$ & 103 \\
\hline 7.72 & 4.34 & 0.36 & 0.18 & 0.34 & & $\mathrm{Cl}$ : nil & 99.71 & 2.766 & Chr. Winther & N. V. Ussing & & 104 \\
\hline 3.87 & 7.52 & tr. & 3.15 & 0.30 & nil & $\mathrm{F}: 0.64$ & $\begin{array}{r}100.34 \\
-0.28 \\
100.06\end{array}$ & & W.H.Herdsmann & (R. Bøgvad) & $\begin{array}{l}\text { Unpub- } \\
\text { lished }\end{array}$ & 105 \\
\hline
\end{tabular}




\begin{tabular}{|c|c|c|c|c|c|c|c|c|c|c|c|c|}
\hline No. & Rock Name & Locality & District & Year & $\mathrm{SiO}_{2}$ & $\mathrm{TiO}_{2}$ & $\mathrm{Al}_{2} \mathrm{O}_{3}$ & $\mathrm{Fe}_{2} \mathrm{O}_{3}$ & $\mathrm{FeO}$ & $\mathrm{MnO}$ & $\mathrm{MgO}$ & $\mathrm{CaO}$ \\
\hline 106 & Granodiorite & $\begin{array}{l}\text { Hodal, Liverpool } \\
\text { Land }\end{array}$ & $\mathrm{NE}$ & 1935 & 55.72 & 1.25 & 17.35 & 3.19 & 2.99 & 0.07 & 3.66 & 7.07 \\
\hline 107 & $\begin{array}{l}\text { Hedenbergite-ande- } \\
\text { sinite }\end{array}$ & $\begin{array}{l}\text { Basistoppen, Kan- } \\
\text { gerdlugssuaq }\end{array}$ & $\mathrm{SE}$ & 1939 & 55.30 & 0.94 & 18.52 & 2.18 & 7.47 & 0.09 & 0.21 & 8.20 \\
\hline 108 & Iron-bearing dolerite & Asuk, Disko & NW & 1879 & 54.80 & & 13.40 & & 14.02 & & 5.33 & 7.84 \\
\hline 109 & Nepheline-porphyry & Akuliarusek & SW & 1911 & 54.58 & $\left.0.62^{1}\right)$ & 20.43 & 2.08 & 3.39 & $\operatorname{tr}$ & $\operatorname{tr}$. & 1.56 \\
\hline 110 & Aegirine-lujavrite & $\begin{array}{l}\text { Laxefjeld, } \\
\quad \text { Kangerdluarsuk }\end{array}$ & SW & 1911 & 53.74 & 0.50 & 14.02 & 10.63 & 1.71 & 0.36 & tr. & 1.18 \\
\hline 111 & Augite-syenite & $\begin{array}{l}\text { Niakornarsuk, } \\
\text { Korok Fjord }\end{array}$ & SW & 1911 & 53.71 & 3.40 & 15.37 & 3.28 & 5.72 & 0.14 & 1.58 & 5.20 \\
\hline 112 & Normalfoyaite & Ivigtut Peninsula & SW & 1911 & 53.54 & nil. & 20.36 & 3.42 & 1.81 & 0.35 & 0.21 & 0.62 \\
\hline 113 & Foyaite & $\begin{array}{l}\text { Igdlerisalik, } \\
\text { Korok Fjord }\end{array}$ & SW & 1911 & 53.53 & 0.44 & 19.69 & 5.09 & 2.83 & 0.24 & nil. & 1.87 \\
\hline 114 & Aegirine-Iujavrite & $\begin{array}{l}\text { Tupersuatsiak } \\
\text { Tunugdliarfik }\end{array}$ & SW & 1911 & 53.44 & 0.30 & 18.64 & 9.38 & 0.86 & 0.10 & nil. & 0.79 \\
\hline 115 & Eklogite & $\begin{array}{l}\text { Hurry Inlet side of } \\
\text { Liverpool Land }\end{array}$ & $\mathrm{NE}$ & 1935 & 53.24 & 0.44 & 5.96 & 5.70 & 5.58 & 0.25 & 16.49 & 10.49 \\
\hline 116 & Arfvedsonite-lujavrite & $\begin{array}{l}\text { Lille Elv, } \\
\text { Kangerdluarsuk }\end{array}$ & SW & 1911 & 53.01 & 0.33 & 15.33 & 9.14 & 4.44 & 0.13 & 0.10 & 0.67 \\
\hline 117 & Iron-bearing dolerite & Mellemfjord, Disko & NW & 1883 & 53.01 & & 15.85 & & $\left.11.53^{*}\right)$ & tr. & 7.51 & 8.72 \\
\hline 118 & $\begin{array}{l}\text { Altered nepheline- } \\
\text { basalt }\end{array}$ & Kangerdlugssuaq & $\mathrm{SE}$ & 1934 & 53.00 & 0.15 & 22.70 & 2.43 & 1.49 & 0.15 & 0.12 & 1.00 \\
\hline 119 & Tremolite-schist & $\begin{array}{c}\text { Uvkusigssat in Lille } \\
\text { Qarajaq Fjord }\end{array}$ & NW & 1905 & 52.74 & 0.29 & 4.35 & 2.35 & 6.58 & 0.12 & 18.29 & 10.86 \\
\hline 120 & $\begin{array}{l}\text { Lamprophyre (kersan- } \\
\text { titic) }\end{array}$ & $\begin{array}{l}\text { Porfyrfjeld, } \\
\text { Canning Land }\end{array}$ & $\mathrm{NE}$ & $\begin{array}{c}(1936) \\
1937\end{array}$ & 52.14 & 1.05 & 12.47 & 0.96 & 6.18 & 0.08 & 6.02 & 7.23 \\
\hline 121 & $\begin{array}{l}\text { Basic hedenbergite- } \\
\text { granophyre }\end{array}$ & $\begin{array}{l}\text { Basistoppen, } \\
\text { Kangerdlugssuaq }\end{array}$ & SE & 1939 & 52.13 & 1.14 & 15.87 & 5.61 & 11.17 & 0.30 & 1.11 & 5.80 \\
\hline
\end{tabular}

*) All iron given as $\mathrm{FeO}$ as no separation between $\mathrm{FeO}$ and $\mathrm{Fe}_{2} \mathrm{O}_{3}$ was undertaken. 


\begin{tabular}{|c|c|c|c|c|c|c|c|c|c|c|c|c|}
\hline $\mathrm{Na}_{2} \mathrm{O}$ & $\mathrm{K}_{2} \mathrm{O}$ & $\mathrm{P}_{2} \mathrm{O}_{5}$ & $\mathrm{H}_{2} \mathrm{O}^{+}$ & $\mathrm{H}_{2} \mathrm{O}^{-}$ & $\mathrm{CO}_{2}$ & Inclusive & Sum & Sp.gr. & Analyst & Published by & Notes & No. \\
\hline 4.23 & 2.64 & 0.88 & 0.52 & 0.25 & & $\begin{array}{l}\mathrm{BaO}: 0.16 \\
\mathrm{~S}: 0.02\end{array}$ & 100.00 & 2.78 & N. Sahlbom & E. H. Kranck & & 106 \\
\hline 6.01 & 0.78 & 0.42 & 0.41 & 0.06 & & & 100.59 & 2.82 & W. A. Deer & $\begin{array}{l}\text { L. R. Wager \& } \\
\text { W. A. Deer }\end{array}$ & & 107 \\
\hline 2 . & 18 & & 1.50 & & & $\begin{array}{l}\mathrm{Ni}: 0.08 \\
\mathrm{Co}: \mathrm{tr} .\end{array}$ & 99.15 & & L. Smith & L. Smith & & 108 \\
\hline 10.70 & 5.74 & tr. & 1.02 & 0.12 & & & 100.24 & 2.698 & Chr. Winther & N. V. Ussing & $\begin{array}{l}\text { 1) Incl. } \\
\mathrm{ZrO}_{2} \text {. }\end{array}$ & 109 \\
\hline 9.02 & 4.77 & nil & 3.40 & & & $\mathrm{ZrO}_{2}: 1.63$ & 100.96 & 2.67 & N. V. Ussing & N. V. Ussing & & 110 \\
\hline 6.84 & 4.11 & 0.52 & 0.45 & 0.33 & & & 100.65 & 2.697 & Chr. Winther & N. V. Ussing & & 111 \\
\hline 10.52 & 4.50 & tr. & 3.85 & 0.20 & nil & $\begin{array}{l}\mathrm{F}: 0.67 \\
\mathrm{~S}: 0.12 \\
\mathrm{Cl}: 0.22 \\
\mathrm{Ba}: \text { nil } \\
\mathrm{Zr}: \frac{\text { nil }}{\text { or tr }} \\
\mathrm{SO}_{3}: 0.04 \\
\mathrm{Li}: \text { tr. }\end{array}$ & $\begin{array}{r}100.43 \\
-0.42 \\
100.01\end{array}$ & & W.H.Herdsmann & (R. Bøgvad) & $\begin{array}{l}\text { Unpub- } \\
\text { lished }\end{array}$ & 112 \\
\hline 9.61 & 5.23 & 0.31 & 0.34 & 0.25 & 0.40 & $\mathrm{Cl}: 0.4$ & $\begin{array}{r}99.87 \\
-0.01 \\
99.86\end{array}$ & 2.751 & Chr. Winther & N. V. Ussing & & 113 \\
\hline 12.10 & 2.43 & nil. & 1.12 & 0.34 & & $\begin{array}{l}\mathrm{ZrO}_{2}: 1.00 \\
\mathrm{Cl}: 0.12\end{array}$ & $\begin{array}{r}100.62 \\
-0.03 \\
100.59\end{array}$ & 2.834 & Chr. Winther & N. V. Ussing & & 114 \\
\hline 0.40 & 0.17 & nil. & 0.40 & 0.10 & & $\begin{array}{l}\mathrm{ZrO}_{2}: \text { nil } \\
\mathrm{S}: \text { nil } \\
\mathrm{F}: \text { nil } \\
\mathrm{BaO}: \text { nil } \\
\mathrm{Cl}: 0.14\end{array}$ & $\begin{array}{r}99.36 \\
0.03 \\
99.33 \\
(100.33)\end{array}$ & 3.363 & N. Sahlbom & T. G. Sahama & & 115 \\
\hline 11.86 & 2.60 & tr. & 1.88 & 0.20 & & $\begin{array}{l}\mathrm{Cl}: 0.23 \\
\mathrm{SO}_{3}: \text { nil }\end{array}$ & $\begin{array}{l}100.57 \\
-0.05 \\
\end{array}$ & 2.844 & Chr. Winther & N. V. Ussing & & 116 \\
\hline 4.49 & tr. & & & & & $\mathrm{ZrO}_{2}: 0.65$ & $\begin{array}{l}100.52 \\
101.11\end{array}$ & & J. Lorenzen & J. Lorenzen & & 117 \\
\hline 10.20 & 2.35 & nil. & 5.90 & 0.35 & nil & S : nil & 99.84 & & W.H.Herdsmann & L. R. Wager & & 118 \\
\hline 0.11 & 0.39 & 0.02 & 3.17 & 0.07 & & $\begin{array}{l}\mathrm{CuO}: 0.07 \\
\mathrm{NiO}: 0.12\end{array}$ & 99.53 & 3.048 & A. Lindner & M. Belowsky & & 119 \\
\hline 1.79 & 2.55 & 0.70 & 3.48 & 0.30 & 4.79 & $\mathrm{BaO}: 0.30$ & 100.04 & & N. Sahlbom & A. Noe-Nygaard & & 120 \\
\hline 3.63 & 1.38 & 0.70 & 0.86 & 0.25 & & & 99.95 & 2.97 & W. A. Deer & $\begin{array}{l}\text { L. R. Wager \& } \\
\text { W. A. Deer }\end{array}$ & & 121 \\
\hline
\end{tabular}




\begin{tabular}{|c|c|c|c|c|c|c|c|c|c|c|c|c|}
\hline No. & Rock Name & Locality & District & Year & $\mathrm{SiO}_{2}$ & $\mathrm{TiO}_{2}$ & $\mathrm{Al}_{2} \mathrm{O}_{3}$ & $\mathrm{Fe}_{2} \mathrm{O}_{3}$ & $\mathrm{FeO}$ & $\mathrm{MnO}$ & $\mathrm{MgO}$ & $\mathrm{CaO}$ \\
\hline 122 & $\begin{array}{l}\text { Kakortokite } \\
\text { (white sheets) }\end{array}$ & $\begin{array}{l}\text { Kringlerne, } \\
\text { Kangerdluarsuk }\end{array}$ & SW & 1911 & 51.62 & 0.44 & 15.63 & 6.06 & 4.98 & 0.33 & tr. & 3.13 \\
\hline 123 & Nepheline-porphyry & Akuliarusek & SW & 1911 & 51.31 & 1.20 & 21.54 & 3.68 & 3.37 & 0.41 & 1.18 & 1.39 \\
\hline 124 & Hornblende-vogesite & $\begin{array}{l}\text { Bastionen, } \\
\text { Ella Island }\end{array}$ & $\mathrm{NE}$ & 1940 & 51.02 & 0.94 & 13.60 & 2.58 & 6.48 & 0.11 & 9.23 & 7.88 \\
\hline 125 & Essexite-porphyrite & Kakarsuak, Narsak & SW & 1911 & 50.98 & 1.38 & 22.15 & 1.04 & 4.25 & tr. & 0.79 & 7.90 \\
\hline 126 & Aegirine-lujavrite & $\begin{array}{l}\text { S. Siorarsuit, } \\
\text { Tunugdliarfik }\end{array}$ & SW & 1911 & 50.72 & $\left.2.84^{1}\right)$ & 15.45 & 11.82 & 0.80 & 0.31 & 0.13 & 0.14 \\
\hline $126 \mathrm{a}$ & $\begin{array}{l}\text { Arfvedsonite-leucite- } \\
\text { tinguaite }\end{array}$ & Kangerdluarsuk & SW & $1898 ?$ & 50.70 & & 13.72 & 6.07 & 7.64 & 1.42 & 0.04 & 0.68 \\
\hline 127 & Iron-bearing dolerite & Fiskernæs & SW & 1883 & 50.64 & & 15.98 & & 14.92 & & 5.14 & 9.39 \\
\hline 128 & Olivine-gabbro & $\begin{array}{l}\text { Tinden, } \\
\text { Kangerdlugsuaq }\end{array}$ & SE & 1939 & 50.41 & 1.75 & 18.30 & 1.96 & 8.13 & 0.16 & 4.25 & 11.20 \\
\hline 129 & Ilimausak-porphyry & Ilimausak & SW & 1911 & 49.64 & 4.25 & 13.74 & 7.10 & 4.97 & 0.03 & 1.58 & 4.88 \\
\hline 130 & Naujaite & Kangerdluarsuk & SW & 1911 & 49.46 & 0.16 & 23.53 & 3.04 & 1.02 & 0.17 & $\operatorname{tr}$. & 0.80 \\
\hline 131 & Kakortokite (red sheets) & $\begin{array}{l}\text { Kringlerne, } \\
\text { Kangerdluarsuk }\end{array}$ & SW & 1911 & 49.39 & 0.49 & 10.39 & 4.31 & 7.72 & 0.97 & nil. & 5.11 \\
\hline 132 & Sodalite-foyaite & $\begin{array}{l}\text { Tupersuatsiak, } \\
\text { Tunugdliarfik }\end{array}$ & SW & 1911 & 49.38 & 0.63 & 17.31 & 4.20 & 5.25 & 0.08 & 0.53 & 2.23 \\
\hline 133 & Dolerite & Brededal, Disko & NW & 1872 & 49.18 & 0.52 & 13.52 & 5.52 & 10.31 & 0.28 & 6.83 & 11.51 \\
\hline 134 & Quartz-gabbro & $\begin{array}{l}\text { Brødretoppen, } \\
\text { Kangerdlugssuaq }\end{array}$ & $\mathrm{SE}$ & 1939 & 49.16 & 3.59 & 15.83 & 4.13 & 8.17 & 0.21 & 4.36 & 10.81 \\
\hline 135 & Dolerite & Uivfaq, Disko & NW & 1879 & 49.02 & & 13.11 & 5.03 & 11.20 & 0.20 & 8.16 & 10.10 \\
\hline 136 & $\begin{array}{l}\text { Kakortokite (black } \\
\text { sheets) }\end{array}$ & $\begin{array}{l}\text { Kringlerne, } \\
\text { Kangerdluarsuk }\end{array}$ & SW & 1911 & 48.90 & & 7.85 & 11.46 & 13.32 & 1.11 & 0.38 & 1.95 \\
\hline 137 & Tholeittic basalt & Cape Dalton & SE & 1934 & 48.89 & 2.64 & 13.60 & 4.72 & 9.65 & 0.24 & 4.54 & 10.09 \\
\hline
\end{tabular}




\begin{tabular}{|c|c|c|c|c|c|c|c|c|c|c|c|c|}
\hline No. & Rock Name & Locality & District & Year & $\mathrm{SiO}_{2}$ & $\mathrm{TiO}_{2}$ & $\mathrm{Al}_{2} \mathrm{O}_{3}$ & $\mathrm{Fe}_{2} \mathrm{O}_{3}$ & $\mathrm{FeO}$ & $\mathrm{MnO}$ & $\mathrm{MgO}$ & $\mathrm{CaC}$ \\
\hline 138 & $\begin{array}{l}\text { Eklogite } \\
\text { (partly converted } \\
\text { into amphibolite) }\end{array}$ & $\begin{array}{l}\text { Hurry Inlet side of } \\
\text { Liverpool Land }\end{array}$ & NE & 1935 & 48.80 & 1.30 & 13.60 & 5.03 & 5.80 & 0.18 & 8.58 & 11.5 \\
\hline 139 & Pigeonite-tholeite & Andrée Land & NE & 1940 & 48.72 & 3.80 & 15.05 & 3.59 & 9.90 & 0.20 & 3.22 & 8.1 \\
\hline 140 & Dolerite & Jackson Island & NE & 1932 & 48.64 & 2.20 & 12.62 & 4.34 & 7.34 & 0.12 & 7.33 & 11.8 \\
\hline 141 & Kaersutite-pegmatite & Østerfjeld, Qaersut & NW & 1928 & 48.50 & 3.05 & 19.41 & 1.07 & 4.67 & 0.11 & 4.26 & 8.9 \\
\hline 142 & $\begin{array}{l}\text { Hornblende-biotite- } \\
\text { schist }\end{array}$ & $\begin{array}{l}\text { Riddarborgen, } \\
\text { Franz Joseph Fjord }\end{array}$ & NE & 1931 & 48.40 & 1.20 & 12.83 & 2.43 & 9.13 & 0.25 & 11.65 & 10.5 \\
\hline 143 & Fayalite-ferrogabbro & $\begin{array}{l}\text { Forbindelses- } \\
\text { gletcher, } \\
\text { Kangerdlugssuaq }\end{array}$ & $\mathrm{SE}$ & 1939 & 48.27 & 2.20 & 8.58 & 4.06 & 22.89 & 0.26 & 1.21 & 7 \\
\hline 144 & Iron-bearing dolerite & Uivfaq, Disko & NW & 1879 & 48.25 & & 14.08 & 5.76 & 10.32 & & 8.39 & 9 \\
\hline 145 & Dolerite & $\begin{array}{l}\text { Kichenpauer Bay, } \\
\text { Clavering Island }\end{array}$ & NE & 1932 & 48.20 & 3.01 & 11.97 & 4.52 & 11.65 & 0.40 & 4.94 & 10 \\
\hline 146 & "Middle" gabbro & $\begin{array}{l}\text { Pukugaqryggen, } \\
\text { Kangerdlugssuaq }\end{array}$ & SE & 1939 & 48.15 & 2.64 & 18.02 & 2.52 & 9.50 & 0.12 & 5.25 & 10.1 \\
\hline 147 & $\begin{array}{l}\text { Basalt, free from } \\
\text { olivine }\end{array}$ & Hare Island & NW & 1918 & 48.10 & 5.68 & 11.22 & 6.94 & 8.93 & 0.23 & 3.78 & \\
\hline 148 & Basalt & Uivfaq, Disko & NW & 1872 & 48.04 & 0.39 & 13.13 & 6.89 & 11.14 & 0.11 & 5.17 & 10.8 \\
\hline 149 & Quartz-gabbro & $\begin{array}{l}\text { Brødretoppen, } \\
\text { Kangerdlugssuaq }\end{array}$ & SE & 1939 & 48.02 & 3.88 & 15.40 & 4.35 & 9.30 & 0.25 & 4.06 & 10 \\
\hline 150 & Dolerite & Uivfaq, Disko & NW & 1879 & 48.02 & $\left.{ }^{1}\right)$ & 15.06 & 6.50 & 9.45 & 0.12 & 8.62 & \\
\hline
\end{tabular}




\begin{tabular}{|c|c|c|c|c|c|c|c|c|c|c|c|c|}
\hline No. & Rock Name & Locality & District & Year & $\mathrm{SiO}_{2}$ & $\mathrm{TiO}_{2}$ & $\mathrm{Al}_{2} \mathrm{O}_{3}$ & $\mathrm{Fe}_{2} \mathrm{O}_{3}$ & $\mathrm{FeO}$ & $\mathrm{MnO}$ & $\mathrm{MgO}$ & Ca \\
\hline 151 & $\begin{array}{l}\text { Olivine-gabbro } \\
\text { (marginal) }\end{array}$ & $\begin{array}{l}\text { Udløberen, } \\
\text { Kangerdlugssuaq }\end{array}$ & SE & 1939 & 48.01 & 1.51 & 19.11 & 1.20 & 8.44 & 0.12 & 7.72 & 10. \\
\hline 152 & $\begin{array}{l}\text { Dolerite, } \\
\text { iron-bearing }\end{array}$ & Uivfaq, Disko & NW & 1879 & 48.00 & $\operatorname{tr}$ & 12.20 & 3.20 & 15.31 & & 8.63 & $9 .^{\prime}$ \\
\hline 153 & Variolitic basalt, altered & $\begin{array}{l}\text { Hængefjeld, } \\
\text { Kangerdlugssuaq }\end{array}$ & $\mathrm{SE}$ & 1939 & 47.89 & 1.74 & 14.36 & 1.63 & 10.14 & 0.19 & 7.28 & 10. \\
\hline 154 & $\begin{array}{l}\text { Olivine-gabbro } \\
\text { (marginal) }\end{array}$ & $\begin{array}{l}\text { East side of Skær- } \\
\text { gaardsbugt, } \\
\text { Kangerdlugssuaq }\end{array}$ & SE & 1939 & 47.83 & 1.29 & 18.62 & 1.16 & 8.87 & 0.09 & 7.92 & 10. \\
\hline 155 & Olivine-basalt & Cape Daussy & $\mathrm{SE}$ & 1934 & 47.81 & 2.51 & 13.64 & 4.50 & 8.84 & 0.22 & 6.51 & 11. \\
\hline 156 & "Diorite" (Ampibolite) & Umanak & NW & 1904 & 47.80 & 1.46 & 18.24 & 0.35 & 9.27 & 0.55 & 8.08 & 11. \\
\hline 157 & $\begin{array}{l}\text { Trachydolerite } \\
\quad \text { (Tasek-porphyrite) }\end{array}$ & Tasek, Narsak & SW & 1911 & 47.79 & 3.82 & 16.88 & 4.66 & 5.92 & tr. & 1.51 & 5. \\
\hline 158 & $\begin{array}{l}\text { Olivine-basalt, } \\
\text { altered }\end{array}$ & $\begin{array}{l}\text { Mikis Fjord, } \\
\text { Kangerdlugssuaq }\end{array}$ & SE & 1939 & 47.61 & 1.58 & 10.92 & 3.80 & 7.25 & 0.19 & 13.49 & \\
\hline 159 & Olivine-basalt & $\begin{array}{l}\text { Barclay Bay, north } \\
\text { side }\end{array}$ & $\mathrm{SE}$ & 1934 & 47.26 & 2.69 & 14.17 & 4.68 & 8.32 & 0.19 & 6.65 & \\
\hline 160 & Dolerite, iron-bearing & Uivfaq, Disko & NW & 1901 & 47.11 & 0.78 & 14.33 & 4.88 & 11.06 & 0.21 & 8.45 & 9. \\
\hline 161 & Prowersite & $\begin{array}{l}\text { Segelsällskapets } \\
\text { Fjord }\end{array}$ & $\mathrm{NE}$ & 1940 & 47.10 & 1.44 & 13.02 & 2.36 & 5.13 & 0.12 & 8.64 & 8. \\
\hline 162 & Olivine-gabbro & $\begin{array}{l}\text { Mellemø, } \\
\text { Kangerdlugssuaq }\end{array}$ & SE & 1939 & 47.01 & 1.47 & 19.12 & 0.71 & 9.12 & 0.08 & 8.98 & 10. \\
\hline
\end{tabular}




\begin{tabular}{|c|c|c|c|c|c|c|c|c|c|c|c|c|}
\hline $\mathrm{a}_{2} \mathrm{O}$ & $\mathrm{K}_{2} \mathrm{O}$ & $\mathrm{P}_{2} \mathrm{O}_{5}$ & $\mathrm{H}_{2} \mathrm{O}^{+}$ & $\mathrm{H}_{2} \mathrm{O}^{-}$ & $\mathrm{CO}_{2}$ & Inclusive & Sum & Sp.gr. & Analyst & Published by & Notes & No. \\
\hline .34 & 0.17 & 0.07 & 0.55 & 0.05 & 0.11 & $\begin{array}{l}\mathrm{S}: 0.29 \\
\mathrm{ZrO}=\text { nil } \\
\mathrm{SrO}_{2}: 0.21 \\
\mathrm{BaO}_{0}: 0.02 \\
\mathrm{Cr}_{2} \mathrm{O}_{3}: \mathrm{tr} \\
\mathrm{CuO}: 0.006 \\
\mathrm{NiO}: \text { nil }\end{array}$ & 100.26 & 2.98 & W. A. Deer & $\begin{array}{l}\text { L. R. Wager \& } \\
\text { W. A. Deer }\end{array}$ & & 151 \\
\hline 1 . & & tr. & 2.25 & & tr. & $\mathrm{S}: \mathrm{tr}$ & 100.45 & & L. Smith & L. Smith & & 152 \\
\hline .01 & 0.22 & 0.18 & 2.76 & 0.10 & 0.19 & $\begin{array}{l}\mathrm{Cl}_{2}: \text { tr. } \\
\mathrm{SrO}: \mathrm{tr} \\
\mathrm{BaO}: \mathrm{tr} \\
\mathrm{Li}_{2} \mathrm{O}: \mathrm{tr} \\
\mathrm{NiO}: \mathrm{tr} \\
\mathrm{S}: \text { tr. }\end{array}$ & 100.20 & 2.99 & H. F. Harwood & $\begin{array}{l}\text { L. R. Wager \& } \\
\text { W. A. Deer }\end{array}$ & & 153 \\
\hline .54 & 0.20 & 0.06 & 0.27 & 0.16 & 0.01 & $\begin{array}{l}\mathrm{S}: 0.25 \\
\mathrm{ZrO}_{2}: \text { nil } \\
\mathrm{SrO}: 0.19 \\
\mathrm{BaO}: 0.02 \\
\mathrm{Cr}_{2} \mathrm{O}_{3}: \text { nil } \\
\mathrm{CuO}: 0.008 \\
\mathrm{NiO}: \text { nil }\end{array}$ & $\begin{array}{c}100.08 \\
(100.07)\end{array}$ & 2.95 & W. A. Deer & $\begin{array}{l}\text { L. R. Wager \& } \\
\text { W. A. Deer }\end{array}$ & & 154 \\
\hline .54 & 0.35 & 0.24 & 0.76 & 1.26 & nil & $\begin{array}{l}\mathrm{BaO}: \text { nil } \\
\mathrm{SrO}: \text { nil } \\
\mathrm{Cl}_{2}: \mathrm{tr} \\
\mathrm{S}: 0.01 \\
\mathrm{Li}_{2} \mathrm{O}: \mathrm{tr} .\end{array}$ & 100.48 & 2.95 & H. F. Harwood & L. R. Wager & & 155 \\
\hline .24 & 0.45 & 0.24 & 0.58 & & & & 100.70 & & W. C. Phalen & W. C. Phalen & & 156 \\
\hline .76 & 3.26 & 0.76 & 1.17 & 0.14 & nil & $\mathrm{Cl}$ : nil & 99.25 & 2.921 & Chr. Winther & N. V. Ussing & & 157 \\
\hline .74 & 0.10 & 0.17 & 3.62 & 0.58 & 0.02 & $\begin{array}{l}\mathrm{Cl}_{2}: 0.02 \\
\mathrm{SrO}: \text { nil } \\
\mathrm{BaO}: \text { nil } \\
\mathrm{LiO}_{2}: \text { tr. } \\
\mathrm{NiO}: 0.06 \\
\mathrm{~S}: \mathrm{tr} .\end{array}$ & 100.22 & 2.95 & H. F. Harwood & $\begin{array}{l}\text { L. R. Wager \& } \\
\text { W. A. Deer }\end{array}$ & & 158 \\
\hline .30 & 0.81 & 0.23 & 1.45 & 0.90 & 0.04 & $\begin{array}{l}\mathrm{Cl}_{2}: 0.01 \\
\mathrm{SrO}: \text { nil } \\
\mathrm{BaO} ; \text { nil } \\
\mathrm{Li}_{2} \mathrm{O}: \mathrm{tr} . \\
\mathrm{S}: 0.01\end{array}$ & 100.35 & 2.97 & H. F. Harwood & L. R. Wager & & 159 \\
\hline .91 & 0.20 & 0.29 & 1.52 & & & $\begin{array}{l}\mathrm{Cl}: 0.09 \\
\mathrm{~S}: 0.53\end{array}$ & 100.58 & & Th. Nicolau & Th. Nicolau & & 160 \\
\hline .16 & 5.85 & 1.56 & 2.27 & 0.34 & $\left.(0.63)^{1}\right)$ & & 100.16 & & N. Sahlbom & A. Rittmann & $\left.{ }^{1}\right)$ Calc. & 161 \\
\hline .33 & 0.28 & 0.06 & 0.24 & 0.09 & & & 100.37 & 3.01 & W. A. Deer & $\begin{array}{l}\text { L. R. Wager \& } \\
\text { W. A. Deer }\end{array}$ & & 162 \\
\hline
\end{tabular}




\begin{tabular}{|c|c|c|c|c|c|c|c|c|c|c|c|c|}
\hline No. & Rock Name & Locality & District & Year & $\mathrm{SiO}_{2}$ & $\mathrm{TiO}_{2}$ & $\mathrm{Al}_{2} \mathrm{O}_{3}$ & $\mathrm{Fe}_{2} \mathrm{O}_{3}$ & $\mathrm{FeO}$ & $\mathrm{MnO}$ & $\mathrm{MgO}$ & $\mathrm{Ca}$ \\
\hline 163 & $\begin{array}{l}\text { Amygdaloidal basalt } \\
\text { (lava) }\end{array}$ & $\begin{array}{l}\text { South side, } \\
\text { Scoresby Sund }\end{array}$ & SE & 1918 & 46.84 & 3.98 & 17.11 & 4.95 & 5.01 & 0.16 & 5.72 & 10. \\
\hline 164 & "Sonnenbrennerbasalt" & Godhavn, Disko & NW & 1927 & 46.75 & 0.88 & 12.85 & 3.64 & 12.68 & & 4.77 & $13 .:$ \\
\hline 165 & Amphibolite & $\begin{array}{l}\text { Cape Swainson, } \\
\text { Liverpool Land }\end{array}$ & $\mathrm{NE}$ & 1935 & 46.74 & 2.88 & 14.77 & 3.11 & 11.00 & 0.18 & 6.52 & 8.4 \\
\hline 166 & Basalt & $\begin{array}{l}\text { Lyngmarksfjeld, } \\
\text { Disko }\end{array}$ & NW & 1933 & 46.58 & 5.18 & 7.14 & 8.57 & 9.65 & & 5.08 & 10.8 \\
\hline 167 & Plagioclase-basalt & Cape Franklin & NE & 1932 & 46.50 & 3.02 & 10.86 & 2.70 & 12.77 & 0.18 & 6.27 & 11.1 \\
\hline 168 & Basalt & $\begin{array}{l}\text { Neills Cliff, } \\
\text { Jameson Land }\end{array}$ & NE & 1931 & 46.44 & 4.63 & 7.31 & 8.52 & 6.59 & & 7.15 & 12. \\
\hline 169 & Dolerite, iron-bearing & Uivfaq, Disko & NW & 1879 & 46.40 & $\operatorname{tr}$. & & 31.75 & & & 8.72 & 10.1 \\
\hline 170 & $\begin{array}{l}\text { Hypersthene-olivine- } \\
\text { gabbro }\end{array}$ & $\begin{array}{l}\text { Uttentals Plateau, } \\
\text { Kangerdlugssuaq }\end{array}$ & $\mathrm{SE}$ & 1939 & 46.37 & 0.79 & 16.82 & 1.52 & 10.44 & 0.09 & 9.61 & 11.2 \\
\hline 171 & $\begin{array}{l}\text { Grønlandite (Hyper- } \\
\text { sthene-hormblendite) }\end{array}$ & Upernivik Island & NW & 1927 & 46.23 & 0.86 & 8.29 & 3.76 & 8.85 & & 19.60 & 8. \\
\hline 172 & Felspar rock & $\begin{array}{l}\text { Mellemø, } \\
\text { Kangerdlugssuaq }\end{array}$ & SE & 1939 & 46.15 & 0.55 & 21.34 & 0.79 & 8.21 & 0.15 & 9.97 & \\
\hline 173 & $\begin{array}{l}\text { Grønlandite (Hyper- } \\
\text { sthene-hornblendite) }\end{array}$ & Upernivik Island & NW & 1927 & 46.13 & 0.82 & 8.17 & 4.00 & 8.71 & & 19.78 & 8 \\
\hline 174 & Essexite & $\begin{array}{l}\text { Panernak Bay, } \\
\text { NW. of Narsak }\end{array}$ & SW & 1911 & 46.10 & 3.34 & 18.59 & 2.63 & 6.68 & 0.05 & 3.23 & 9.8 \\
\hline 175 & Basalt & $\begin{array}{l}\text { Lyngmarksfjeld, } \\
\text { Disko }\end{array}$ & NW & 1931 & 46.05 & 3.02 & 13.83 & 5.56 & 6.85 & & 7.65 & 10. \\
\hline 176 & Basalt & $\begin{array}{l}\text { Lyngmarksfjeld, } \\
\text { Disko }\end{array}$ & NW & 1931 & 45.91 & 4.36 & 8.75 & 8.06 & 9.11 & 0.16 & 5.63 & 10. \\
\hline 177 & "Middle" gabbro & $\begin{array}{l}\text { Pukugaqryggen, } \\
\text { Kangerdlugssuaq }\end{array}$ & SE & 1939 & 45.65 & 2.59 & 15.08 & 3.41 & 14.86 & 0.15 & 6.35 & 9. \\
\hline 178 & $\begin{array}{l}\text { Eklogite } \\
\text { (Strongly amphiboli- } \\
\text { tized) }\end{array}$ & Liverpool Land & $\mathrm{NE}$ & 1935 & 45.56 & 2.60 & 16.10 & 5.59 & 9.15 & 0.20 & 6.54 & 8.6 \\
\hline
\end{tabular}




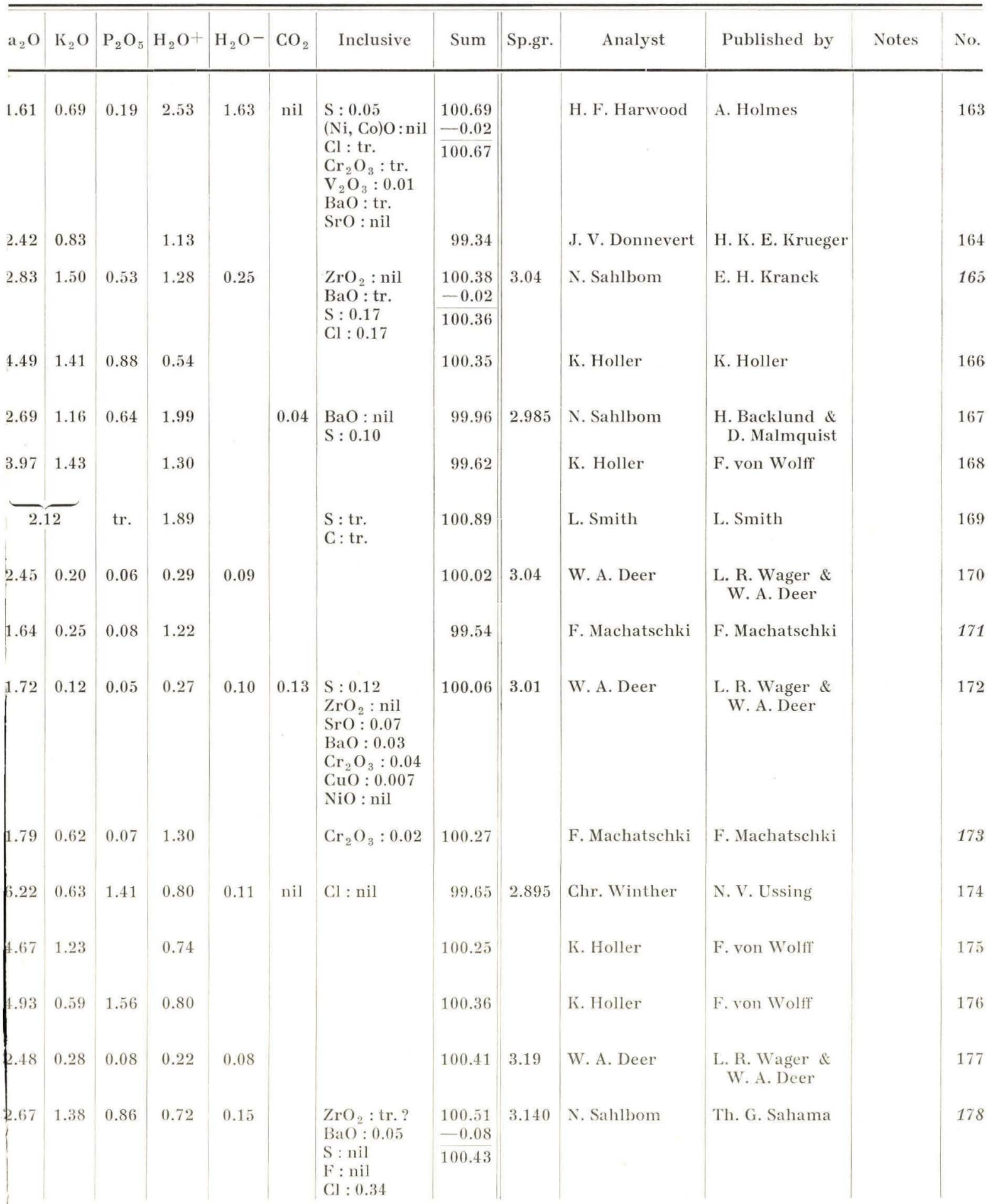




\begin{tabular}{|c|c|c|c|c|c|c|c|c|c|c|c|c|}
\hline No. & Rock Name & Locality & District & Year & $\mathrm{SiO}_{2}$ & $\mathrm{TiO}_{2}$ & $\mathrm{Al}_{2} \mathrm{O}_{3}$ & $\mathrm{Fe}_{2} \mathrm{O}_{3}$ & $\mathrm{FeO}$ & $\mathrm{MnO}$ & $\mathrm{MgO}$ & $\mathrm{Cal}$ \\
\hline 179 & Olivine-free gabbro & $\begin{array}{l}\text { Ivnarmiut, } \\
\text { Kangerdlugssuaq }\end{array}$ & SE & 1939 & 45.51 & 3.96 & 14.36 & 5.04 & 12.65 & 0.20 & 5.38 & 9.9 \\
\hline 180 & $\begin{array}{l}\text { Hypersthene-olivine } \\
\text { gabbro }\end{array}$ & $\begin{array}{l}\text { Uttentals Plateau, } \\
\text { Kangerdlugssuaq }\end{array}$ & SE & 1939 & 45.48 & 0.94 & 16.41 & 2.09 & 9.29 & 0.06 & 11.65 & 10.4 \\
\hline 181 & Trachydolerite & Nunasarnausak & SW & 1911 & 45.27 & 4.41 & 15.03 & 4.04 & 9.10 & tr. & 6.59 & 6.6 \\
\hline 182 & Fayalite-ferrogabbro & $\begin{array}{l}\text { Basistoppen, } \\
\text { Kangerdlugssuaq }\end{array}$ & SE & 1939 & 45.19 & 1.67 & 9.37 & 5.78 & 23.77 & 0.32 & 0.43 & 9.0 \\
\hline 183 & "Anortitfels" & Uivfaq, Disko & NW & 1872 & 44.94 & & 22.20 & & 9.45 & & 4.98 & 11.0 \\
\hline 184 & $\begin{array}{l}\text { Hortonolite-ferro- } \\
\text { gabbro }\end{array}$ & $\begin{array}{l}\text { Basishusene, } \\
\text { Kangerdlugssuaq }\end{array}$ & $\mathrm{SE}$ & 1939 & 44.81 & 2.55 & 13.96 & 3.75 & 16.66 & 0.17 & 5.54 & 8.5 \\
\hline 185 & $\begin{array}{l}\text { Ferrohortonolite-ferro- } \\
\text { gabbro }\end{array}$ & $\begin{array}{l}\text { Basistoppen, } \\
\text { Kangerdlugssuaq }\end{array}$ & SE & 1939 & 44.61 & 2.43 & 11.70 & 2.05 & 22.68 & 0.21 & 1.71 & 8.7 \\
\hline 186 & $\begin{array}{l}\text { Nepheline-tephritic } \\
\text { rock }\end{array}$ & $\begin{array}{l}\text { Cape Fletcher, } \\
\text { Canning Land }\end{array}$ & $\mathrm{NE}$ & 1907 & 44.43 & 1.40 & 17.89 & 4.00 & 4.94 & tr. & 2.40 & 12.6 \\
\hline 187 & Olivine basalt & $\begin{array}{l}\text { Umîvik Fjord, } \\
\text { Svartenhuk }\end{array}$ & NW & 1931 & 44.42 & 1.92 & 13.61 & 3.19 & 9.67 & 0.20 & 9.25 & $14.4^{\prime}$ \\
\hline 188 & Olivine-trachybasalt & $\begin{array}{l}\text { Mt. Nordhoek, } \\
\text { Loch Fyne }\end{array}$ & NE & 1932 & 44.16 & 2.41 & 15.18 & 3.64 & 9.40 & 0.13 & 9.24 & 10.5 \\
\hline 189 & Fayalite-ferrogabbro & $\begin{array}{l}\text { Basistoppen, } \\
\text { Kangerdlugssuaq }\end{array}$ & SE & 1939 & 44.13 & 2.48 & 7.88 & 4.05 & 26.63 & 0.48 & 0.25 & 10.0 \\
\hline 190 & $\begin{array}{l}\text { Dolerite } \\
\text { (sill in peridotite) }\end{array}$ & Osterfjeld, Qaersut & NW & 1928 & 44.10 & 2.76 & 15.24 & 7.34 & 7.85 & 0.15 & 4.54 & 7.3 \\
\hline 191 & Basalt & Uivfaq, Disko & NW & 1870 & 44.01 & & 14.27 & 14.75 & 3.89 & & 8.11 & 10.9 \\
\hline 192 & $\begin{array}{l}\text { "Olivinestone", } \\
\text { disintegrated }\end{array}$ & Siorarsuit & SW & 1884 & 43.81 & & 3.29 & & 10.31 & & 38.13 & tr. \\
\hline 193 & Albite-diabase & $\begin{array}{l}\text { Mt. Ramsay, } \\
\text { Gauss Peninsula }\end{array}$ & $\mathrm{NE}$ & 1932 & 43.69 & 4.01 & 10.43 & 5.32 & 12.69 & 0.18 & 6.04 & $3.9^{\prime}$ \\
\hline 194 & Naujaite & $\begin{array}{l}\text { Nunasarnak } \\
\text { Tunugdliarfik, }\end{array}$ & SW & 1911 & 43.39 & 0.20 & 23.13 & 3.62 & 3.24 & tr. & nil & 0.5 \\
\hline
\end{tabular}




\begin{tabular}{|c|c|c|c|c|c|c|c|c|c|c|c|c|}
\hline $\mathrm{ia}_{2} \mathrm{O}$ & $\mathrm{K}_{2} \mathrm{O}$ & $\mathrm{P}_{2} \mathrm{O}_{5}$ & $\mathrm{H}_{2} \mathrm{O}^{+}$ & $\mathrm{H}_{2} \mathrm{O}^{-}$ & $\mathrm{CO}_{2}$ & Inclusive & Sum & Sp.gr. & Analyst & Published by & Notes & No. \\
\hline 2.43 & 0.35 & 0.32 & 0.12 & 0.10 & & & 100.33 & 3.22 & W. A. Deer & $\begin{array}{c}\text { L. R. Wager \& } \\
\text { W. A. Deer }\end{array}$ & & 179 \\
\hline 2.06 & 0.27 & 0.05 & 0.77 & 0.26 & & & 99.79 & 3.00 & W. A. Deer & $\begin{array}{l}\text { L. R. Wager \& } \\
\text { W. A. Deer }\end{array}$ & & 180 \\
\hline 5.07 & 1.08 & 0.16 & 1.85 & 0.14 & 0.38 & $\begin{array}{l}\mathrm{Cl}: \mathrm{tr} . \\
\mathrm{SO}_{3}: \text { nil }\end{array}$ & 99.76 & 2.988 & Chr. Winther & N. V. Ussing & & 181 \\
\hline 2.43 & 0.49 & 0.91 & 0.57 & 0.31 & & & 100.29 & 3.30 & W. A. Deer & $\begin{array}{l}\text { L. R. Wager \& } \\
\text { W. A. Deer }\end{array}$ & & 182 \\
\hline 1.86 & 0.06 & & $\mathrm{H} ?$ : & $\left.0.31^{1}\right)$ & & $\begin{array}{l}\left.\mathrm{C}^{1}\right): 3.35 \\
\mathrm{Fe}: 1.11 \\
\mathrm{Cl}: 0.20 \\
\mathrm{~S}: \mathrm{tr} . \\
\mathrm{Cu}: \mathrm{tr} .\end{array}$ & 99.47 & 2.927 & Nauckhoff & $\begin{array}{l}\text { E. G. R. } \\
\text { Nauckhoff }\end{array}$ & $\begin{array}{l}\left.{ }^{1}\right) \text { A mean } \\
\text { of two } \\
\text { determ. }\end{array}$ & 183 \\
\hline 3.35 & 0.33 & 0.08 & 0.34 & 0.19 & & & 100.26 & 3.18 & W. A. Deer & $\begin{array}{l}\text { L. R. Wager \& } \\
\text { W. A. Deer }\end{array}$ & & 184 \\
\hline 2.95 & 0.35 & 1.85 & 0.22 & 0.20 & 0.04 & $\begin{array}{l}\mathrm{S}: 0.31 \\
\mathrm{ZrO}_{2}: 0.01 \\
\mathrm{SrO}: 0.08 \\
\mathrm{BaO}: 0.02 \\
\mathrm{Cr}_{2} \mathrm{O}_{3}: \mathrm{tr} . \\
\mathrm{CuO}: 0.016 \\
\mathrm{NiO}: \mathrm{tr} .\end{array}$ & 100.15 & 3.22 & W. A. Deer & $\begin{array}{c}\text { L. R. Wager \& } \\
\text { W. A. Deer }\end{array}$ & & 185 \\
\hline 2.55 & 3.02 & & 5.16 & & 1.09 & & 99.48 & & N. Sahlbom & O. Nordenskjöld & & 186 \\
\hline 1.01 & 0.65 & 0.26 & 1.14 & 0.33 & & $\begin{array}{l}\mathrm{Cr}_{2} \mathrm{O}_{3}: 0.09 \\
\mathrm{NiO}: 0.03\end{array}$ & $\begin{array}{c}100.26 \\
(100.35)\end{array}$ & & H. Nieland & H. Nieland & & 187 \\
\hline 2.12 & 1.27 & 0.40 & 1.52 & & nil & $\mathrm{BaO}$ : nil & 100.05 & 2.926 & N. Sahlbom & $\begin{array}{l}\text { H. Backlund \& } \\
\text { D. Malmquist }\end{array}$ & & 188 \\
\hline 2.15 & 0.47 & 1.61 & 0.30 & 0.19 & & & 100.65 & 3.39 & W. A. Deer & $\begin{array}{l}\text { L. R. Wager \& } \\
\text { W. A. Deer }\end{array}$ & & 189 \\
\hline 2.69 & 1.17 & 0.50 & 2.54 & 2.71 & 0.57 & & 99.51 & 2.760 & Schäffer & $\begin{array}{r}\text { F.K. Drescher \& } \\
\text { H.K.E.Krueger }\end{array}$ & & 190 \\
\hline 2.61 & 0.97 & & & & & & 99.52 & & Th. Nordström & $\begin{array}{l}\text { Th. Nordström } \\
\text { (A. E. Norden- } \\
\text { skjöld) }\end{array}$ & & 191 \\
\hline 0.1 & & & 1.41 & & & $\begin{array}{l}\mathrm{NiO}: \mathrm{tr} . \\
\text { Foreign mat- } \\
\text { ter }: 2.37\end{array}$ & 99.45 & & K. Rørdam & K. Rørdam & & 192 \\
\hline 0.77 & 4.90 & 0.87 & 3.59 & 0.60 & 3.72 & & $\begin{array}{c}100.78 \\
(100.77)\end{array}$ & & N. Sahlbom & H. G. Backlund & & 193 \\
\hline 9.68 & 1.51 & nil & 1.36 & 0.21 & & $\begin{array}{l}\mathrm{ZrO}_{2}: 0.27 \\
\mathrm{Cl}: 3.63 \\
\mathrm{SO}_{3}: \text { nil }\end{array}$ & $\begin{array}{r}100.80 \\
-0.82 \\
99.98\end{array}$ & 2.545 & Chr. Winther & N. V. Ussing & & 194 \\
\hline
\end{tabular}




\begin{tabular}{|c|c|c|c|c|c|c|c|c|c|c|c|c|}
\hline No. & Rock Name & Locality & District & Year & $\mathrm{SiO}_{2}$ & $\mathrm{TiO}_{2}$ & $\mathrm{Al}_{2} \mathrm{O}_{3}$ & $\mathrm{Fe}_{2} \mathrm{O}_{3}$ & $\mathrm{FeO}$ & $\mathrm{MnO}$ & $\mathrm{MgO}$ & $\mathrm{CaC}$ \\
\hline 195 & Ankaramite & $\begin{array}{l}\text { Ladder Mountain, } \\
\text { head of Musk Ox } \\
\text { Fjord }\end{array}$ & $\mathrm{NE}$ & 1932 & 43.20 & 3.60 & 8.57 & 5.00 & 6.24 & 0.14 & 12.00 & 15.5 \\
\hline 196 & Basalt, iron-bearing & Uivfaq, Disko & NW & 1872 & 42.72 & tr. & 16.01 & 1.64 & 14.27 & $\operatorname{tr} . ?$ & 7.93 & 10.1 \\
\hline 197 & Peridotite var. picrite & Qaersut, Nûgssuaq & NW & 1904 & 42.63 & & 6.88 & 3.33 & 7.27 & 0.36 & 29.36 & 5.9 \\
\hline 198 & Olivine-basalt & Hare Island & NW & 1918 & 42.57 & 5.07 & 14.40 & 3.54 & 8.39 & 0.10 & 6.78 & 11.7 \\
\hline 199 & Basalt & $\begin{array}{l}\text { Lyngmarksfjeld, } \\
\text { Disko }\end{array}$ & NW & 1931 & 41.60 & 4.33 & 5.90 & 11.43 & 10.71 & 0.07 & 6.81 & 11.9 \\
\hline 200 & Gabbro-picrite & $\begin{array}{l}\text { Uttentals plateau, } \\
\text { Kangerdlugssuaq }\end{array}$ & SE & 1939 & 41.27 & 1.54 & 8.71 & 2.69 & 10.52 & 0.16 & 27.09 & \\
\hline 201 & $\begin{array}{l}\text { Basalt, fragments in the } \\
\text { weathered breccia }\end{array}$ & Uivfaq, Disko & NW & 1872 & 41.25 & 0.34 & 13.06 & 16.18 & 10.78 & 0.25 & 6.41 & $7.9^{\prime}$ \\
\hline 202 & Basalt & $\begin{array}{l}\text { Lyngmarksfjeld, } \\
\text { Disko }\end{array}$ & NW & 1931 & 41.18 & 4.04 & 12.40 & 7.47 & 6.89 & 0.35 & 5.15 & 11 \\
\hline 203 & $\begin{array}{l}\text { Felspar-bearing } \\
\text { peridotite }\end{array}$ & Osterfjeld, Qaersut & NW & 1928 & 41.11 & 0.61 & 8.34 & 2.99 & 7.19 & tr. & 29.32 & \\
\hline 204 & Dolerite, iron-bearing & Uivfaq, Disko & NW & 1879 & 41.06 & tr. & 10.20 & 8.32 & 21.01 & & 8.76 & \\
\hline 205 & Basalt & $\begin{array}{l}\text { Lyngmarksfjeld, } \\
\text { Disko }\end{array}$ & NW & 1931 & 40.97 & 2.84 & 14.44 & 4.17 & 6.81 & 0.30 & 6.97 & 12 \\
\hline 206 & Soapstone & Unortok Fjord & SW & 1874 & 40.91 & & 11.41 & 5.37 & 6.04 & & 25.92 & \\
\hline 207 & "Anortitfels" & Uivfaq, Disko & NW & 1883 & 39.75 & & 26.08 & 12.33 & & & 4.51 & 12. \\
\hline 208 & "Olivinestone" & Siorarsuit & SW & 1884 & 39.17 & & 3.30 & nil & 10.56 & & 43.46 & $\operatorname{tr}$ \\
\hline 209 & $\begin{array}{l}\text { "Augitzüge", in } \\
\text { peridotite }\end{array}$ & Østerfjeld, Qaersut & NW & 1928 & 38.48 & 1.20 & 4.36 & 4.69 & 10.12 & tr. & 29.54 & \\
\hline 210 & "Anortitfels" & Uivfaq, Disko & NW & 1872 & 37.92 & & 32.36 & & $\left.4.02^{1}\right)$ & 0.19 & 2.86 & $11.5^{\circ}$ \\
\hline
\end{tabular}




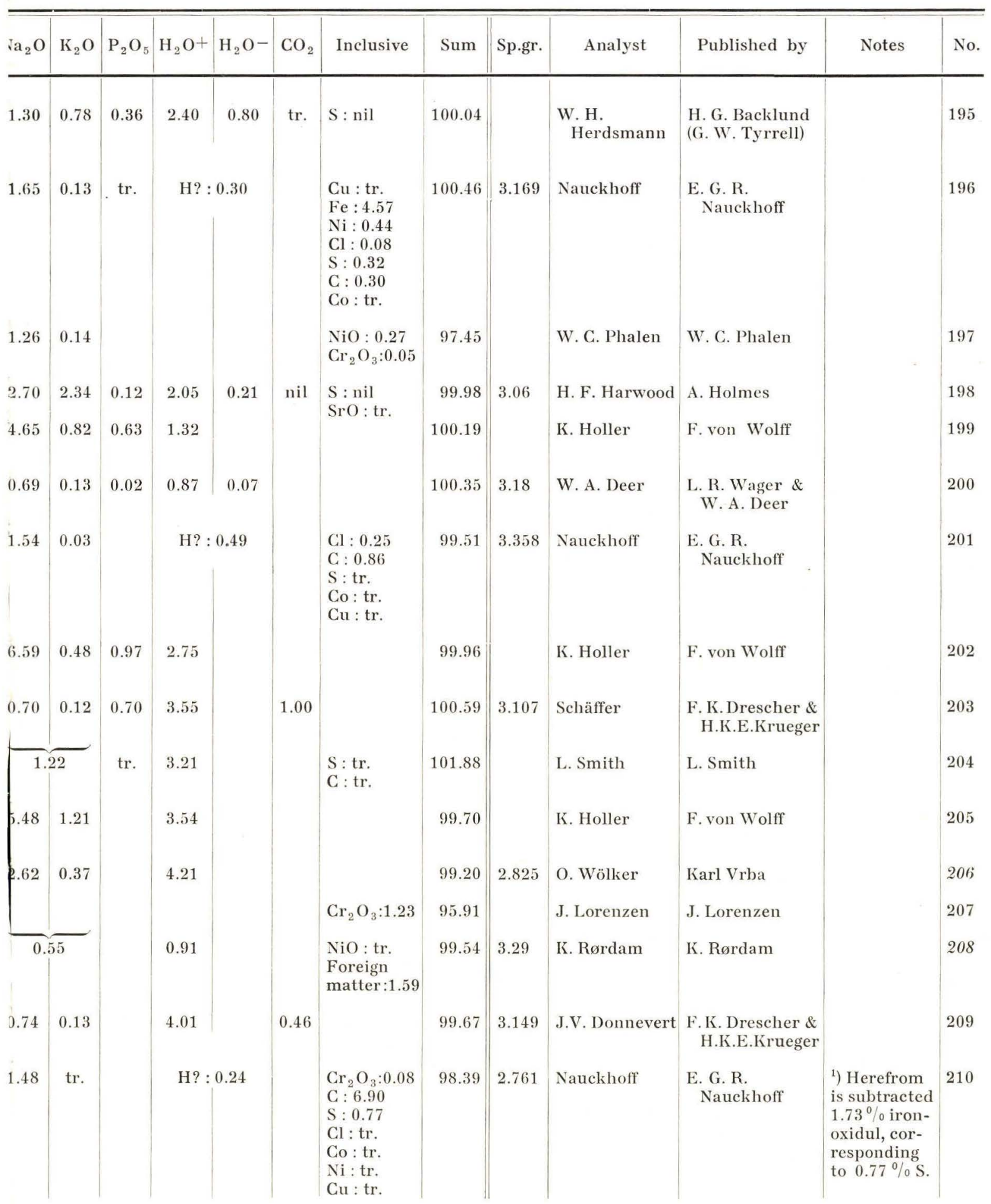




\begin{tabular}{|c|c|c|c|c|c|c|c|c|c|c|c|c|}
\hline No. & Rock Name & Locality & District & Year & $\mathrm{SiO}_{2}$ & $\mathrm{TiO}_{2}$ & $\mathrm{Al}_{3} \mathrm{O}_{3}$ & $\mathrm{Fe}_{2} \mathrm{O}_{3}$ & $\mathrm{FeO}$ & $\mathrm{MnO}$ & $\mathrm{MgO}$ & $\mathrm{CaC}$ \\
\hline 211 & "Mandelsteinkern" & $\begin{array}{l}\text { Lyngmarksfjeld, } \\
\text { Disko }\end{array}$ & NW & $\begin{array}{l}1933 \\
-34\end{array}$ & 36.86 & 3.60 & 13.75 & 10.94 & 2.31 & 0.15 & 6.21 & 10.0 \\
\hline 212 & "Anortitfels" & Uivfaq, Disko & NW & 1872 & 36.59 & & 19.18 & & 14.85 & 0.29 & 7.24 & 8.7: \\
\hline 213 & Alnöite & Liverpool Land & $\mathrm{NE}$ & 1907 & 36.29 & 4.60 & 10.91 & 7.55 & 5.52 & 0.23 & 9.29 & 13.4 \\
\hline 214 & "Anortitfels" & Uivfaq, Disko & NW & 1872 & 34.72 & & 31.83 & 4.88 & $\left.5.53^{1}\right)$ & & 9.35 & 10.1 \\
\hline 215 & Magnesite-pyroxenite & Narsak & SW & 1911 & 31.77 & 12.97 & nil & 12.97 & 10.23 & tr. & 15.77 & 12.2 \\
\hline 216 & Soapstone & $\begin{array}{l}\text { Loc. unknown. } \\
\text { (Prof. Laube got it } \\
\text { in Lichtenau) }\end{array}$ & SW & 1873 & 30.32 & & 17.90 & $=$ & 7.71 & & 29.88 & 1.2 \\
\hline 217 & $\begin{array}{l}\text { Soapstone, } \\
\text { same specim. as } 216\end{array}$ & " & SW & 1873 & 29.82 & & 17.96 & & 7.47 & & 29.40 & 1.2 \\
\hline $218^{*}$ & "Bauxitähnliche Hülle" & $\begin{array}{l}\text { Lyngmarksfjeld, } \\
\text { Disko }\end{array}$ & NW & $\begin{array}{r}1933 \\
-34\end{array}$ & 25.59 & 5.06 & 24.04 & 18.71 & & 0.65 & 0.81 & 2.9 \\
\hline
\end{tabular}




\begin{tabular}{|c|c|c|c|c|c|c|c|c|c|c|c|c|}
\hline $\mathrm{Na}_{2} \mathrm{O}$ & $\mathrm{K}_{2} \mathrm{O}$ & $\mathrm{P}_{2} \mathrm{O}_{5}$ & $\mathrm{H}_{2} \mathrm{O}^{+}$ & $\mathrm{H}_{2} \mathrm{O}^{-}$ & $\mathrm{CO}_{2}$ & Inclusive & Sum & Sp.gr. & Analyst & Published by & Notes & No. \\
\hline 5.45 & 0.57 & 0.40 & 9.18 & & & & 99.43 & & K. Holler & K, Holler & & 211 \\
\hline 0.79 & tr. & & H? : & 0.31 & & $\begin{array}{l}\mathrm{C}: 2.55 \\
\mathrm{Fe}: 5.01 \\
\mathrm{Ni}: 0.25 \\
\mathrm{Cl}: 0.23 \\
\mathrm{~S}: \mathrm{tr} . \\
\mathrm{Co}: \mathrm{tr} . \\
\mathrm{Cu}: \mathrm{tr} .\end{array}$ & 96.02 & 3.141 & Nauckhoff & $\begin{array}{l}\text { E. G. R. } \\
\text { Nauckhoff }\end{array}$ & & 212 \\
\hline 3.42 & 1.40 & & 3.65 & & 3.85 & & 100.19 & & N. Sahlbom & $\begin{array}{l}\text { O. Norden- } \\
\text { skjöld }\end{array}$ & & 213 \\
\hline 1.00 & 0.27 & & H? : & 0.29 & & $\begin{array}{l}\mathrm{C}: 0.53 \\
\mathrm{Fe}: 0.09 \\
\mathrm{Cl}: 0.12\end{array}$ & 98.80 & 2.942 & Nauckhoff & $\begin{array}{l}\text { E. G. R. } \\
\text { Nauckhoff }\end{array}$ & $\begin{array}{l}\text { 1) From } \mathrm{FeO} \\
\text { found } \\
\text { through } \\
\text { titration is } \\
\text { subtracted } \\
0.09 \% \mathrm{Fe}\end{array}$ & 214 \\
\hline 2.69 & 0.54 & & & 0.05 & & & 99.79 & & & & & 215 \\
\hline & & 0.11 & 12.28 & & & $\begin{array}{l}\mathrm{F}: \mathrm{tr} . \\
\mathrm{SO}_{3}: \mathrm{tr} .\end{array}$ & 99.48 & 2.702 & J. Janovský & J. V. Janovský & & 216 \\
\hline & & & & & & $\begin{array}{l}\mathrm{F}: \mathrm{tr} . \\
\mathrm{SO}_{3}: \mathrm{tr} .\end{array}$ & 85.87 & & J. Janovský & J. V. Janovský & & 217 \\
\hline 4.63 & 0.45 & & 17.08 & & & & 99.96 & & K. Holler & K. Holler & & $218^{*}$ \\
\hline
\end{tabular}




\section{Description of Rocks analyzed.}

\section{Denmark.}

1. Alminding granite. Precambrian. Bjergbakke, Vestermarie, Bornholm. Callisen, K. (1932 (1934), p. 89).

A middle-grained, streaked, light reddish-grey rock, rather rich in quartz, irregularly defined and with undulatory extinction and microcline, often with perthitic structure; myrmekite is common, the plagioclase is an oligoclase $(17 \%$ an). Of the dark minerals an olivebrown or greenish biotite is predominant, then follow sphene and ore; hornblende is sparse. Accessories are orthite and a little fluorite.

2. Hammer granite. Precambrian.

Hammeren, Bornholm.

KALB, G. (1913, p. 52).

A middle-grained, light grey rock with a reddish tinge. Macroscopically seen to contain felspar, quartz and violet fluorite. U. M. ${ }^{1}$ ): Main constituents: Quartz, often undulatory and micropegmatitically intergrown with potash-felspar and plagioclase. The potash-felspar is microcline and is mostly developed as microperthite. The plagioclase is oligoclase-albite. Minor constituents: Biotite, sphene and a little ore. Sphene often forms an edge round the ore, the apatite and the zircon.

3. Hammer granite. Precambrian.

Hammeren, Bornholm.

Callisen, K. (1928, p. 18, 1932 (1934), p. 97).

Compare no. 2. Main constituents: Microcline-perthite, undulatory quartz and acid oligoclase (core $25 \%$ an, edge $10 \%$ an). Other constituents: Olive-brown biotite common, hornblende sparse, ore, and sphene; orthite is more common in this granite than in the other granite varieties of Bornholm.

1) »U. M. is used here and throughout this paper as abbreviation for under microscope. 
4. Granite. Precambrian.

Allinge, Bornholm.

RøRDAM, K. (1914, p. 307).

No mineral composition given in the text; reference is made to Cohen \& Deecke, Greifswald 1899: Úber das krystalline Grundgebirge der Insel Bornholm.

5. Svaneke granite. Precambrian.

Ibs Kirke, Bornholm.

Callisen, K. (1932 (1934), p. 113).

A very coarse-grained rock, rather rich in dark minerals, which are often present in heaps. The quartz is somewhat undulatory, the main constituent is microcline-perthite, the microcline of which is perfectly fresh, while the perthitic plagioclase is somewhat altered. The plagioclase is an oligoclase $(25-27 \%$ an) occurring in centimetre-large grains; myrmekite is common. An olive or greenish biotite is predominant among the dark constituents; there are also bluish-green hornblende, much sphene and apatite, epidote, fluorite and seldom chalcopyrite and hematite.

6. Vang granite. Precambrian.

"At pegmatite«, Vang, Bornholm.

KaLB, G. (1913, p. 59).

The rock analyzed was taken from a zone with a marked parallel structure at a pegmatitic vein (comp. no. 7, and description of »Hauptgranit« on pp. 54-55). U. M.: Albite-oligoclase is most frequent, oligoclase recedes and the potash-felspar is perthitic, further quartz, green hornblende and biotite, the latter of which predominates, sphene and ore.

7. Vang granite, (»Klondyke»). Precambrian.

Rødkløv, south of Vang, Bornholm.

KaLB, G. (1913, p. 57).

The mineral description originates from »Hauptgranit《 on Ringebakken, but the granite from R $\phi$ dkløv did not show any differences (according to G. KaLB). Greenish hornblende and biotite often grown together form dark clumps accompanied by sphene and ore. The zoned plagioclase consists of a core of oligoclase-andesine, and myrmekite is developed where it borders on potash-felspar. The potashfelspar is micrographically intergrown with quartz, which is less abundant than the felspar.

8. Ronne granite. Precambrian.

Klippegaard, Bornholm.

Rørdaм, K. (1890, p. 75).

Megascopically greyish green felspar, quartz and dark green and black hornblende are observed. U. M. are further seen: Biotite, apatite, zircon and iron-oxydes. The felspars are orthoclase, microcline and plagioclase. Sphene is absent, as is fluorite. 
9. Paradisbakke granite. Precambrian.

Paradisbakkerne, Bornholm.

KALB, G. (1913, p. 62).

A dark, streaked rock, consisting of fine-grained biotite, hornblende, sphene, potash-felspar and quartz. The large plagioclase grains are only seldom idiomorphic, but often poikiliticly intergrown by quartz. They are zonary with the core consisting of oligoclase, the edge of albite-oligoclase.

10. Rønne granite. Precambrian.

Klippegaard, Bornholm.

Callisen, K. (1932 (1934), p. 39).

The main constituents are: Quartz, microcline (microcline-perthite) seldom orthoclase, plagioclase $(28-20 \%$ an), when zonary developed with edges of albite (ab. $10 \%$ an), but usually the plagioclase is surrounded by microcline-perthite in parallel intergrowth, green strongly pleocroic hornblende $\left(2 \mathrm{E}=75^{\circ}\right)$, brown biotite with $\alpha$ : light yellow $\gamma$ : dark brown (lepidomelane). Accessories: Much apatite, sphene, zircon, ore.

Secondary minerals: Muscovite (a little), calcite, epidote and chlorite.

11. Rønne granite (Knudsbakke granite). Precambrian.

Rønne area, Bornholm.

KALB, G. (1913, p. 67).

A dark grey xenomorphic-granular rock, the dark colour due to large quantities of hornblende and biotite. Sphene is rare, ore and apatite common. Plagioclases vary from oligoclase-andesine to albiteoligoclase; they are seldom idiomorphic and always surrounded by parallel-grown potash-felspar. The potash-felspar is almost always, the plagioclase not so often intergrown with quartz.

12. Rønne granite (Knudsbakke granite). Precambrian.

"At pegmatite«, Rønne area, Bornholm.

KALB, G. (1913, p.68).

Compare no. 15.

The rock is a strongly banded variety of the Rønne granite, taken near a pegmatite vein.

13. Volcanic ash-layer no. $+\mathbf{1 9}$. Eocene.

Ejerslev, Island of Mors, Nord-Jylland.

BøGGILD, O. B. (1918, p. 25). 
Colour of ash grey. The glassy particles have a greyish or brownish colour, pores are common, elongated in one direction; refringence 1.510. Among the minerals in the ash felspars predominate, single augite grains are also met with. The felspars are dominatingly oligoclase-albite (plagioclases with higher refringence are not common) or orthoclase.

14. Volcanic ash-layer no. + 19. Eocene.

Island of Fur, Nord-Jylland.

Norin, RolF (1940, p. 42).

Compare description of no. 13.

15. Rønne granite. Precambrian.

$800 \mathrm{~m}$ east of Store Almegaard, Bornholm.

Callisen, K. (1932, (1934), p. 57 $\left.)^{1}\right)$.

The rock originates from the belt of transition at St. Almegaard. The structure is porphyric and somewhat banded. There is less biotite and hornblende than in the Rønne granite proper (cf. no. 10), and more sphene and ore. The phenocrysts consist of plagioclase and in single cases of microcline.

16. Voleanic ash-layer no. - 17. Eocene.

Limf jord area, Nord-Jylland.

BøGGILD, O. B. (1918, p. 21).

Colour of ash varies from yellowish-white (Hanklit, Stolleklint), to light grey (Fur) and light brown (Ejerslev). The glass grains possess a characteristic greenish-grey colour in their centre, the borders being colourless; the refringence decreases from the centre outwards as determined by the values of 1.538 and 1.500 respectively. Of minerals the felspars, acid plagioclase (albite) and orthoclase are present; there also occur numerous grains of a brownish hornblende, pleochroic with $\alpha$ olive-brown and $\gamma$ reddish-brown and a few grains of augite (brownish from Fur and Ejerslev, green from Fur and Hanklit).

17. Volcanic ash-layer no. - 12. Eocene.

Hanklit, Thy, Nord-Jylland.

BøgGILD, O. B. (1918, p. 23).

Colour of ash greyish to black. Composition basaltic, although some oligoclase-albite is found. No augite occurs. The refringence of the glassy grains varies, the maximum value being 1.607 .

$\left.{ }^{1}\right)$ The same analysis was published in an incorrect form in 1928 (26 p. 18. no. 9). 
18. Volcanic ash-layer no. $+\mathbf{1 9}$. Eocene.

Helgenæs, Djursland, Øst-Jylland.

Norin, Rolf (1940, p.42).

Compare description of no. 13.

19. Volcanic ash-layer no. -12. Eocene.

Fur, Nord-Jylland.

Norin, Rolf (1940, p. 40).

Compare description of no. 17.

20. Volcanic ash-layer no. + 19. Eocene.

Ølst, Øst-Jylland.

Norin, Rolf (1940, p. 43).

Compare description of no. 13.

21. Volcanic ash-layer no. - 12. Eocene.

Ølst, Øst-Jylland.

Norin, Rolf (1940, p. 39).

Compare description of no. 17.

22. Volcanic ash-layer no. + 101. Eocene.

Hanklit, Thy, Nord-Jylland.

Norin, RolF (1940, p.38).

No mineral description given by Norin; the following data are found on p. 27 in BøgGILD (1918): »Colour of ash dark, composition basaltic $\ll$.

23. Volcanic ash-layer no. + 101. Eocene.

Fur, Nord-Jylland.

NORIN, ROLF (1940, p. 39).

Compare description of no. 22.

24. Volcanic ash-layer no. + 101. Eocene.

Helgenæs, Djursland, Øst-Jylland.

NORIN ROLF (1940, p. 38).

Compare description of no. 22.

\section{The Faeroes.}

25. »Trapp anhydré«. Tertiary.

Strømø.

Durocher, M. J. (1841, p. 563).

Rock description not of much use to day, being a hundred years old. 
26. Augite-andesite. Tertiary.

Kolter.

Osann, A. (1884, p. 49).

The fresh, greyish-brown, fine-grained rock is filled with cavities containing opal and heulandite. The rock contains no phenocrysts, and no olivine was found. The pyroxene is diallag-like $\left.{ }^{1}\right)$. Some brown, globulite-filled glass is recorded.

27. Non-porphyritic basalt. Tertiary.

Vaag Fjord, Suðerø.

Holmes, A. (1918, p. 201).

A dark, compact, grey-green rock. U. M. micro-phenocrysts of labradorite in glomerophyritic aggregation, in a sub-ophitic groundmass consisting of labradorite-laths and granular plates of pale-green, slightly pleochroic enstatite-augite. Well-shaped and skeletal crystals of ilmenite are very abundant. There is a small amount of residual glass, sometimes brown and carrying globulites, sometimes green and showing incipient crystallization. The composition in vol- $\%$ is the following: Labradorite (+ inclusions) $45 \%$, pyroxene $39 \%$, magnetite and ilmenite $12 \%$, glass $4 \%$.

28. Trapp. Tertiary.

Height west of Torshavn, Strømø.

Streng, A. (1853, p. 110).

Unaltered trapp with small pores and of a greenish-grey colour. But for felspar the mineral constituents are not clearly distinguishable. The pores may be empty or filled with a greenish clayey substance.

28a. Big-felspar basalt. Tertiary.

Velbestad, western side of Strømø.

WALKer, F. \& C. F. DAVIDSON (1936, p. 887).

The felspar phenocrysts extend up to $1 \frac{1}{2}$ inches in length and form about $28 \%$ of the total volume of the rock. Composition of phenocrysts bytownitic, occasionally with a thin border of andesine. The matrix contains elongated laths, $0.2 \mathrm{~mm} \times 0.4 \mathrm{~mm}$ of plagioclase in sub-ophitic relationship with pale brown augite in grains up to $0.4 \mathrm{~mm}$. Further occur a considerable amount of chlorophaeite and serpentine. The mineralogical composition by volume is: Plagioclase $45.1 \%$, augite $27.8 \%$, chlorophaeite and serpentine $22.6 \%$ and ironores $4.5 \%$.

29. Olivine-dolerite. Tertiary.

Summit of Froðbiarkambur above Tværaa, Suðerø.

Walker, F. \& C. F. Davidson (1936, p. 886, no. D.).

$\left.{ }^{1}\right)$ The augite was separately analyzed (cf. 38. p. 48). 
A non-porphyritic fine-grained olivine-dolerite. Laths of plagioclase $\left(\mathrm{ab}_{1} \mathrm{an}_{2}\right), 0,2 \mathrm{~mm} \times 0,05 \mathrm{~mm}$, are in marked ophitic relationship with fawn-coloured pyroxene which forms clumps up to $1 \mathrm{~mm}$ in diameter. Olivine is fairly abundant in grains up to $1 \mathrm{~mm}$ in length which are sometimes idiomorphic, but more often penetrated subophitically by plagioclase. Much of the olivine is altered to chlorophaeite. Irregular crystals of iron ore, interstitial zeolites (including analcite and doubly-refracting varieties) and brown glass are the most abundant accessories. The mineral composition by volume is: Plagioclase $45,8 \%$, olivine $16,9 \%$, pyroxene $30,7 \%$, iron ores $5,6 \%$, zeolites $1,0 \%$.

30. Basalt with porphyritic felspars. Tertiary.

Torshavn, Strømø.

Washington, H. S. (1922, p. 786, no. 1).

A slightly vesicular basalt, showing rather numerous thick, tabular phenocrysts of labradorite in a densely aphanitic, dark grey groundmass. The texture is porphyritic, sub-ophitic. There are no phenoerysts of either pyroxene or olivine. The groundmass is made up in great part of small tables of labradorite, which are multiply twinned and have a composition not quite as calcic as $a b_{1} a n_{2}$. Between these, in less amount, are small anhedral grains, rather than true ophitic areas, of a colourless augite, with very few and small grains of olivine, very rare grains of ore, and some small prismoids of apatite. There is a considerable interstitial, somewhat cloudy, glass, but the rock appears to be very fresh.

31. Zeolitic amygdaloidal rock. Tertiary. Height west of Torshavn. Strømø.

STRENG, A. (1853, p. 110).

The groundmass is fine-grained. The colour of the rock is greenishbrown. Among the zeolites chabasite is recorded.

32. „Trapp hydraté«. Tertiary.

Height west of Torshavn. Strømø.

Durocher, M. J. (1841, p. 559).

Rock-description not of much use to day, being a hundred years old.

33. Zeolitic amygdaloidal rock. Tertiary.

Height west of Torshavn. Strømø.

Streng, A. (1853, p. 111).

A greatly altered reddish-brown, zeolite-filled amygdaloidal rock with chabasite and desmine in veins. 
34. Basalt with porphyritic felspars. Tertiary.

Höyvík, Strømø.

Simpson, J. B. (1928, p. 516, no. 3).

The felspar phenocrysts or glomero-porphyritic aggregates attain an average greatest length of from 5 to $7 \mathrm{~mm}$. Chlorophaeite in large lumps is common. The composition of the plagioclase-phenocrysts is basic labradorite-bytownite, the groundmass-plagioclase is slightly less basic. The laths have here an average lenght of $0,5 \mathrm{~mm}$, and the granular aggregates of pale or fawn-coloured augite are correspondingly large. A little olivine is present, and a marked quantity of isotropic substance, dark-brown or ollve-green in colour (probably chlorophaeite). Iron-ore is not conspicuous. There is no real ophitic texture in the rock.

35. Olivine-basalt (non-porphyritic). Tertiary.

Trangisvaag, Suðerø.

Simpson, J. B. (1928, p. 516, no. 1).

A fine to medium-grained olivine-basalt. Serpentinized olivine is present as small $(0,2 \mathrm{~mm})$ crystals in a matrix of granular augite and labradorite. The augite shows a slight tendency to crystallize in two generations; the larger individuals are sub-idiomorphic and display a very faint purplish tint, which is absent, or cannot be detected in the smaller anhedral granules; these are colourless or fawn. The felspar laths average less than $0,5 \mathrm{~mm}$ in greatest length and there is an occasional larger crystal or glomeroporphyritic aggregate. Analcite in notable amount is apparent, as is also glass with a dark brown border and lighter yellow interior. Iron-ore is present.

36. Ultramafic olivine-basalt. Tertiary. Southeast of church, Ejde, Østerø.

Walker, F. \& C. F. Davidson (1936, p. 889, no. H.).

A zeolite-bearing, olivine-rich, ophitic dolerite. The olivine occurs as elongated, idiomorphic crystals, usually about $3 \mathrm{~mm} \times 0,5 \mathrm{~mm}$ in size, and as small grains of irregular outline $0,5 \mathrm{~mm}$ in diameter, which may have floated off from larger crystals. The axial angle is near to $90^{\circ}$. Twinning on the long axis is not infrequently seen, and minute inclusions of black spinellides are very common. A remarkable feature is the freshness of the olivines, which are only rarely pseudomorphed in iddingsite or green serpentine. A pale greenishbrown monoclinic pyroxene with high axial angle occurs in marked ophitic relationship with plagioclase and forms clumps up to $2 \mathrm{~mm}$ in diameter. Occasionally small olivines are included poikiliticly. Plagioclase (mainly $\mathrm{ab}_{2} \mathrm{an}_{3}$ ) forms elongated laths (breadth/length ratio $1 / \mathrm{s}$ ) and has undergone a certain amount of zeolitisation. The zeolites themselves occur interstitially and in veins. Iron ore is scarce. The mineral composition by volume is: Plagioclase $36,9 \%$, olivine $29,5 \%$, augite $25,4 \%$, iron ores $1,1 \%$ and zeolites $7,1 \%$. 
37. Altered palagonite-tuff. Tertiary.

Height west of Torshavn. Strømø.

Streng, A. (1853, p. 111).

An altered »palagonite-tuff « which graduates into a clayey substance. In the palagonite matter areas of zeolites and areas of decomposed rock occur. The total-colour is greyish-brown.

\section{Greenland.}

38. Sanidine-rhyolite. Tertiary.

NE. About $10 \mathrm{~km}$ west of Cape Franklin, Gauss Peninsula. Backlund, H. G. \& D. Malmquist (1935, p. 67 no. 1486).

Intensely silicified. In a greyish-green groundmass only few quartzphenocrysts are to be seen; the felspar-phenocrysts are almost wiped out. The groundmass is spotted with ore, carries some sericite and is reticulated by veins of needle-like quartz-crystals.

39. Sanidine-rhyolite. Tertiary.

NE. About $10 \mathrm{~km}$ west of Cape Franklin, Gauss Peninsula. Horizontal laccolite (?).

Backlund, H. G. \& D. MalmQuist (1935, p. 67, no. 1480).

The rock contains automorphic phenocrysts of sanidine, a little albite and quartz. The groundmass is a rather coarse microgranite and contains some sericite. Vol-\%: Quartz-phenocrysts 1,6\%, felsparphenocrysts $13,2 \%$ and groundmass $85,2 \%$.

40. Psammitic gneiss. Metamorphic complex.

NE. Hurry Inlet side of Liverpool Land.

Kranck, E. H. (1935, p. 34).

Quartz, plagioclase $\left(\mathrm{an}_{20}\right)$, orthoclase, biotite and small quantities of a green hornblende. The accessories are: Magnetite, sphene, apatite and very little zircon.

41. Alkali-rhyolite with sanidine-phenocrysts. Devonian. Base of lava-flow.

NE. Northwest of Cape Graah, north coast of Ymer Island.

Rittmann, A. (1940, p. 44, no. 463 a).

Phenocrysts of quartz partly in well-defined crystals, partly corroded, and sanidine which in places is still quite fresh. Pseudomorphs with calcedony and bluish-green hornblende with some yellowish-green chlorite may have originated from pyroxene, probably aegirine-augite. The groundmass is hyalopilitic with flow-texture; the original glassy parts have been replaced by a rather coarse aggregate of quartz with random orientation which in a poikilitic manner encloses microliths of orthoclase. 
42. Phenocryst-poor alkali-rhyolite. Devonian.

NE. South side of Musk Ox F jord.

Rittmann, A. (1940, p. 47, no. 168).

Some of the quartz-phenocrysts, more or less corroded, are surrounded by a quartz border interwoven poikiliticly by orthoclase; others lie directly in the dominatingly felsitic groundmass. Sanidine, only locally perthitic, is also found as phenocrysts. The groundmass shows fluidal texture with light and dark bands; the light bands are of coarser grain than the dark ones and show transitions to poikilitic devitrification-structure; the darker bands are devitrified, the texture being felsitic. The composition by volume is: Quartzphenocrysts $1,4 \%$, sanidine-phenocrysts $1,1 \%$, groundmass $97,5 \%$.

43. »Quartz-keratophyre $\left.{ }^{1}\right)$. Devonian $\left.{ }^{2}\right)$.

NE. North slope of Mt. Ramsay, Gauss Peninsula.

Backlund, H. G. (1932, Min. descr., p. 53, anal. p. 96, no. 1236).

The microgranophyric groundmass is now and then rather coarse and shows needle-like intergrowth of quartz with faint reddish dotted felspar. When the granophyre individuals decrease in dimensions they are in areas replaced by micro-spherulites; these are also found along early cracks. The quartz-phenocrysts show all stages of resorbtion in spite of sharp outlines and are generally encircled by a granophyric halo of the same orientation as the central quartz individual. The felspar is in part a coarse orthoclase-perthite, in which the albite component sometimes replaces the orthoclase component so that only skeleton-like parts are left (the albite is multiply twinned, while the mother-mineral only rarely shows Carlsbadtwinning) in part albite occurring as a primary constituent, with only weakly developed twinning and generally intensely sericitized. Ore forms irregular lumps and grains in the groundmass; a little zircon also occurs. Ferrous lumps within well defined limits may originate from a pyribole (aegirine?).

44. Hypabyssal granophyre-granite ${ }^{3}$ ). Devonian $\left.{ }^{4}\right)$.

NE. Southeast foot of Mt. Högbom, Gauss Peninsula.

Backlund, H. G. (1932, Min. descr. p. 48-49, anal. p. 96, no. 189).

The mineral constituents are pure albite and potash-felspar, both brick-red. The albite is idiomorphic with polysynthetic twins with lamels of almost same width; albite-twins predominate, periclinetwins occur. The potash-felspar is an orthoclase-perthite with irregular occurrence of the perthite member; its outer part is intergrown by quartz-micropegmatite. Micropegmatite forms a groundmass

1) Backlund (1932, p. 53): "Quartz-keratophyre«, Rittmann (1940, p. 37-38): »Alkalirhyolite " or "quartz-porphyry ".

2) By Backlund (1932) considered as belonging to the sub-Cambrian evolution phase, by Bütler (1935) and Rittmann (1940) shown to be of Devonian age.

$\left.{ }^{3}\right)$ By Rittmann (1940), classified as runitic aplite-granite.

$\left.{ }^{4}\right)$ Rittmann (1940, p. 61). 
between the two felspars, and now and then also occurs as a core in the centre of the potash felspar. The larger, rounded quartz grains in the groundmass have generally the same orientation as neighbouring $\gg$ quartz-fishes $\ll$ in the micropegmatite. No mafic constituents were recorded, but for a few lumps of ore. A little accessory muscovite was observed.

45. Granite. Metamorphic complex (Precambrian).

NW. Umanak Island.

Phalen, W. C. $(1904$, p. 187 , no. 75,480$)$.

Of medium grain and with pinkish hue; texture hypautomorphic granular. The mineral content is that of the gneiss (no. 69) even to the merest accessories and alteration products, but the proportions vary, especially that of biotite.

46. Acid orthoclase-bearing porphyry. Palaeozoic (Devonian?).

NE. Cape Fletcher, Canning Land. Cobble from the sea-shore. NoRdenskJöld, O. (1907, p. 199).

A brick-red porphyry wih small inconspicuous porphyritic crystals, among which felspar is predominant, though in certain areas a larger quantity of quartz stands out. U. M. numerous little dihexaedra of quartz are seen; the larger individuals are composed of felspar, predominantly of plagioclase which is intensely weathered and kaolinized. The groundmass is microgranitic.

47. Acid granophyre. Tertiary.

SE. West ridge of Tinden, Kangerdlugssuaq. Large sill (upper part).

Wager, L. R. \& W. A. Deer (1939, p. 208, no. 3058).

Consists of small rectangular crystals of acid plagioclase which pass into a fine-grained micropegmatite, while interstitially a little quartz not intergrown wih felspar is developed. A dirty brown mineral, in small flakes and associated with a little iron-ore is probably stilpnomelane; it is widely scattered but not so abundant as the almost colourless chlorite which occurs interstitially with calcite and quartz.

48. Quartz-rhyolite. Tertiary.

NE. About $10 \mathrm{~km}$ west of Cape Franklin, Gauss Peninsula.

Semi-laccolite.

Analysis first publ. by Backlund (1932, p. 96, no. 1490), anal. repeated and furnished with description of rock in BACKLUND \& Malmquist (1935, p. 32).

Groundmass micropoikilitic with some sericite-alteration; a weak calcite-alteration can also be traced, contains some accessory hematite. The vol- $\%$ of the constituents are: Quartz-phenocrysts 7,3\%, sanidinephenocrysts $10,2 \%$ and groundmass $82,4 \%$. Single grains of zircon, sphene, tourmaline and apatite occur. 
49. Alkali-granite.

SW. Ivigtut Peninsula.

(Unpublished analysis, submitted to us by R. BøgVAD).

No rock-description.

50. Red granite with aplitic groundmass (Porphyry-granite). Precambrian.

NE. Cape Oswald, Ella Island. Ball from late-Precambrian tillite. Backlund, H. G. (1932, p. 96, no. P. 10).

The texture is somewhat porphyric, the main constituent being automorphic orthoclase-perthite; next comes quartz, which shows signs of automorphy. The interspaces are filled by quartz and albite with aplitic texture. There are also green chlorite with ore and rutile grains, locally with muscovite and remnants of dark brownish biotite. Chlorite with some epidote and with reddish carbonate-rim may be pseudomorphs on pyroxene. Accessories are apatite, zircon and ore.

51. Sanidine-rhyolite. Tertiary.

NE. About $7 \mathrm{~km}$ west of Cape Franklin, Gauss Peninsula. Sill or sheet.

Backlund, H. G. \& D. MalmQuist (1935, p.67, no. 1510).

The mineral composition in vol-\%: Quartz-phenocrysts 2,0\%, felspar-phenocrysts $9,1 \%$ and groundmass $88,9 \%$.

The original perthite (phenocrysts) is intensely pigmented. Small quartz-»drusen « contain sericite and now and then calcite.

52. Quariz-porphyry (Comendite). Age unknown, probably Precambrian.

SW. N. Siorarsuit, $830 \mathrm{~m}$ above sea-level east of summit of Ilimausak. $5 \mathrm{~m}$ wide dyke.

Ussing, N. V. (1911, p. 224, no. 24).

The rock is rather altered. U. M. the felspar-phenocrysts are seen to consist of an irregular microperthitic intergrowth of potash-felspar and albite; phenocrysts of quartz and ferromagnesian minerals, now quite converted into iron oxides, also occur. The extremely finegrained groundmass consists of orthoclase-microperthite mixed with crystals of pure albite and contains small dots of irregular patches of black iron oxide. A few grains of zircon and a little fluorite were also observed.

53. Spherulitic rhyolite. Tertiary.

NE. About $6 \mathrm{~km}$ west of Cape Franklin, Gauss Peninsula. Lava flow.

Backlund, H. G. \& D. MalmQuist (1935, p. 55, no. 1501).

The microgranitic groundmass makes up 81,6 vol- $\%$ and the phenocrysts 18,4 vol- $\%$ of the rock. The phenocrysts are the following: Quartz 1,8\%, sanidine $14,5 \%$ and plagioclase $2,1 \%$. 
54. Greyish-brown porphyry without phenocrystic quartz. Palaeozoic (?).

NE. Mt. Nordenskjöld, head of Nathorst Fjord. Ball from Eotriassic conglomerate.

NoE-NygaARD, A. ((1936), 1937, p. 112, no. 60 a).

The rock is thought by N-N to be related to the palaeozoic magmatites of Canning Land. It is a hard, almost chertlike porphyry with a groundmass of a dark greyish-brown colour and with small pinkish felspar-phenocrysts.

55. Red granite. Post-Devonian $\left.(?)^{1}\right)$.

NE. Mt. La Cour, Gauss Peninsula.

Backlund, H. G. (1932, Min. descr. p. 55, anal. p. 101, no. 211).

A uniform brick-red rock with big arcuated felspars and greyishwhite quartz fillings which are younger than the deformation that the rock has undergone. U. M. the rock shows intense kataclastic deformation. The plagioclase is a pure albite $(0-5 \%$ an $)$ with fine albite-twinning; pericline twins are rare. The albite is filled with quartz-microbreccia between the thin lamellae, as is also the case with the potash-felspar, an irregular perthite, but here the lamellae are broader. The mode of occurrence of quartz and felspar makes it probable that they were once in micropegmatitic intergrowth, i. e. the rock was once a granophyre. Ore is sparse, so is zircon. A little calcite belongs to a later epoch.

56. Spherulitic rhyolite. Tertiary.

NE. About $10 \mathrm{~km}$ west of Cape Franklin, Gauss Peninsula. Semi-laccolite.

Analysis first published by BAcklund, H. G. (1932, p. 96 - by mistake numbered as 1492); published again with description of mineral content by Backlund, H. G. \& D. MalmQuist (1935, p. 31, no. 1508).

The volumetric analysis gives the following composition: Groundmass: Spherulitic part $33,8 \%$, microgranitic part $53,4 \%$. Phenocrysts: Quartz $2,1 \%$, plagioclase $1,9 \%$, sanidine $8,8 \%$.

57. Two-mica-granite. Metamorphic Complex (Caledonian).

NE. South side of Kempe Fjord.

BACKLUND, H. G. (1932, p. 91, no. 1328).

A light grey to white two-mica-granite, with potash affinities, which carries quartz with undulatory extinction, potash-felspar, albitic plagioclase and a dull brown biotite. Needles of sillimanite are found in the quartz. Myrmekite is well developed.

1) Rittmann, A. (1940 p. 38 and p. 67-68). 
58. Two-mica-granite. Metamorphic Complex (Caledonian).

NE. Forsblad Fjord.

Backlund, H. G. (1932, p. 91, no. 1349).

A light grey to white two-mica-granite, with potash affinities, which carries quartz with undulatory extinction, potash-felspar, albitic plagioclase and reddish-brown biotite. Myrmekite is well developed.

59. Two-mica-granite. Metamorphic Complex (Caledonian).

NE. North coast of Clavering Island.

BackLund, H. G. (1932, p. 91, no. 39).

Pink-dotted two-mica-granite with potash affinities. The constituents are microcline with coarse lattice and weakly perthitic plagioclase which is idiomorphic in long prisms - often zoned (oligoclase-albite), biotite of dull dark-brown colour, muscovite and quartz with undulatory extinction.

60. Two-mica-granite. Metamorphic Complex (Caledonian).

NE. Forsblad Fjord.

Backlund, H. G. (1932, p. 91, no. 1351).

Pink-dotted two-mica-granite with potash affinities. The constituents are microcline with coarse meshes and of weakly perthitic habit, idiomorphic oligoclase-albite, somewhat zoned, reddish-brown biotite, muscovite and undulatory quartz.

61. Soda-granite. Age unknown, probably Precambrian.

SW. Iviangussat, Kangerdluarsuk.

Ussing, N. V. (1911, p. 114, no. 2).

The soda-granite in zones from $1 / 2-2 \mathrm{~m}$ width surrounds fragments of sandstone in augite-syenite, due to a process of absorbtion. Both mineralogically and chemically it bears close resemblance to the arfvedsonite-granite of Ilimausak. It varies from coarse to fine-grained. U. M. the constituents are: Perthitic microcline-albite, quartz, catophorite-like hornblende and aegirine-augite (often with a little aegirine). Accessories are zircon and ore. (The sample analyzed was light grey, rather fine-grained and without aegirine-augite).

62. Fine-grained granite. Metamorphic Complex. (Devonian?).

NE. Hurry Inlet, Liverpool Land.

Kranck, E. H. (1935, p. 87).

As to mineral-content, compare rock no. 65 .

63. Spherulite from spherulitic rhyolite. Tertiary.

NE. About $6 \mathrm{~km}$ west of Cape Franklin, Gauss Peninsula. Lava flow.

Backlund, H. G. \& D. MalmQuist (1935, p. 55, no. 1504). 
Component minerals are stated to form the following vol-\%: Phenocrystic quartz $0,9 \%$, phenocrystic felspar $1,7 \%$, spherulite-quartz $28,3 \%$, spherulite-groundmass $48,2 \%$, calcite and sericite $20,9 \%$.

64. Chlorite-oligoclase-microcline-gneiss. Metamorphic Complex. NE. From the centre of the anticline, Musk Ox Fjord inlier.

Wisemann, I. D. H. (1931, p. 323, no. 62).

Chlorite is the distinctive mineral; it occurs in independant plates or aggregates of two or three individual plates. Rutile in a sagenitic web, sphene, iron-ores and occasionally calcite occur as inclusions in the chlorite. White mica is sometimes intergrown with the chlorite. The felspars include acid oligoclase and microcline. Quartz is moderately abundant; orthite in fairly large crystals, apatite, zircon and a little magnetite also occur.

65. Coarse porphyric granite. Metamorphic Complex.(Devonian?). NE. Hurry Inlet, Liverpool Land.

Kranck, E. H. (1935, p. 87).

The following mineral content is recorded: Perthitic orthoclase, plagioclase (core $44 \%$ an, border $28 \%$ an), quartz, yellowish-brown biotite and sometimes also muscovite and the accessories: apatite, zircon and ore.

66. Arfvedsonite-granite. Age unknown, probably Precambrian. SW. South of highest summit of Ilimausak.

Ussing, N. V. (1911, p. 114, no. 1).

The fresh rock is coarse-grained and of a light greenish colour. The diameter of the felspar grains varies from $2-4 \mathrm{~mm}$; the quartz grains are a little smaller, and the arfvedsonite-anhedra $10-15 \mathrm{~mm}$. U.M. the following constituents are revealed: Alkali-felspar (perthitic microcline-albite), quartz, arfvedsonite, enigmatite and a little aegirine. Occasional constituents are: Zircon, pyrochlore (?) and elpidite (?). The secondary constituents, magnetite (titano-magnetite), fluorite, hematite, ferric hydrates and calcite occur in the altered varieties.

67. Biotite-granite. Palaeozoic (probably Devonian).

NE. North side of Cape Wardlaw, Canning Land.

NOE-NygaARD, A. ((1936), 1937, p. 39, no. 368).

A medium-grained, hypautomorphic granular granite with the following constituents: Quartz $31,7 \%$, biotite 6,7\%, potashfelspar 31,3 vol- $\%$, plagioclase 30,2 vol- $\% 1$ ) and accessories 0,1 vol- $\%$.

The accessories are: Apatite, sphene, zircon, pyrite and black ore.

$\left.{ }^{1}\right)$ The values given here are taken from an »errata list not supplied with all copies of the paper (The values of table 1 , lit. no. 31 are 44,6 and 16,9 vol-\% respectively, these being incorrect owing to faulty tracing of some intensely sericite-filled felspars).

Instead of line 3 and 4 in table 1 on p. 28 (31) read:

$\begin{array}{llllllll}\text { Kalifeldspat } & \ldots \ldots \ldots & 34,8 \% & 32,0 \% & 31,0 \% & 31,3 \% & 20,0 \% & 13,2 \% \\ \text { Plagioklas } & \ldots \ldots \ldots & 21,9 \% & 27,7 \% & 26,9 \% & 30,2 \% & 25,9 \% & 39,9 \%\end{array}$


68. Granite. Metamorphic Complex. NE. Ättestupan, Kejser Franz Joseph Fjord.

BACKLUND, H. G. (1932, p. 91, no. 1424).

The rock consists of microcline (with Carlsbad-twinning) often without twinning-net, oligoclase-albite (13\% an), quartz (undulatory) and dark olive-brown biotite which dominates over muscovite. The rock contains veins filled with iron-rich epidote (omitted in the material analyzed).

69. Gneiss. Metamorphic Complex (Precambrian).

NW. Umanak Island.

Phalen, W. C. $(1904$, p. 185 , no. 75,478).

A typical gneissoid rock consisting of roughly alternating layers of quartz with felspar and biotite. The texture is hypautomorphic granular. The constituents are: Albite, oligoclase, orthoclase, microcline, quartz, biotite and the accessories: apatite, zircon and sphene. In the felspars the alteration-products sericite and kaolin occur.

70. Grey gneiss. Metamorphic Complex (Precambrian). NW. Siusasigsak, north of Jakobshavn.

Belowsky, M. (1905, p. 24).

A grey, fine-grained rock of evident gneissic habit, consisting of orthoclase, plagioclase, quartz and biotite. Microcline, albite, epidote and kornerupite have been formed through recrystallisation. The accessories are: Ore, zircon, sphene, apatite, epidote and tourmaline. The rock is considered to have originated from granite.

71. Microcline-granite. Metamorphic Complex. (Caledonian). NE. Storefjord, Liverpool Land.

Kranck, E. H. (1935, p. 81).

The constituents are: Quartz 23,51 vol- $\%$, microcline 27,02 vol- $\%$, oligoclase 42,04 vol- $\%$, biotite 5,82 vol- $\%$, sphene 1,19 vol- $\%$ and magnetite 0,42 vol- $\%$.

72. Rhyolitic eruption-breccia. Tertiary.

NE. About $10 \mathrm{~km}$ west of Cape Franklin, Gauss Peninsula. Sheet or flow.

Backlund, H. G. \& D. MalmQuist (1935, p. 73, no. 1476).

Parts of a subophitic mass into which enter partly fragmented quartz and intensely sericite-altered sanidine-phenocrysts, passes diffusely into a dense, microfelsitic, sericite-filled substance of rhyolitic character; this mass contains quartz and often rather big, profoundly altered, perthitic sanidine-phenocrysts. The groundmass is thought to be a rather heterogeneous mixture of basaltic and rhyolitic material. 
73. Grey, fine-grained gneiss, Precambrian.

NE. Spiral Creek, Cape Weber, Andrée Land. Tillite-ball from late Precambrian tillite.

BACKLund, H. G. (1932, p. 104, no. P. 9).

The main felspar is a perthitic microcline; an albitic plagioclase also occurs. Quartz is present as elongated, irregular areas with undulatory extinction. Remnants of a pale brown biotite are met with, but the greater part of this mineral is converted into chlorite of a dull greenish colour and pigmented with grains of ore and lenses of calcite. Accessories are: Apatite, orthite and ore.

74. Grey gneiss. Metamorphic Complex.

SE. $100 \mathrm{~m}$ west of contact, Mellemø, Kangerdlugssuaq.

Wager, L. R. \& W. A. Deer (1939, p. 10, no. 1867).

A fairly fine-grained, granulitized gneiss mainly consisting of quartz and plagioclase with vague layers of green ferromagnesian minerals and pink bands rich in orthoclase. Mode: Quartz $28 \%$, orthoclase $15 \%$, plagioclase $49 \%$ and biotite $8 \%$.

[An analysis of the alkalies in a similar rock from the Kangerdlugssuaq area (no.1865) gave the following result (op. cit. p. 10) $: \mathrm{Na}_{2} \mathrm{O}=$ $3,98 \%$ and $\mathrm{K}_{2} \mathrm{O}=2,93 \%$.]

75. Red phorphyry with phenocrystic quartz. Palaeozoic(?).

NE. Tvekegledal, Wegener Peninsula. Conglomerate-ball from Eotriassic deposits.

Noe-NygaARd, A. ((1936), 1937, p. 112, no. 375 c).

The conglomerate-ball is thought by $\mathrm{N}-\mathrm{N}$ to be related to the palaeozoic magmatites of Canning Land, NE.

The rock is a somewhat weathered, coarse-grained, red porphyry. The following vol- $\%$ are found among the phenocrystic minerals: Quartz $35,5 \%$, felspars $66,0 \%$ and biotite \& accessories $0,5 \%$.

76. Quartz-monzonite. Metamorphic Complex. Precambrian.

NW. Nûgssuaq Peninsula, eastern part.

Phalen, W. C. $(1904$, p. 208, no. 75,491).

The texture of the rock is pan-automorphic. The constituents are: Oligoclase, which is intensely kaolinized, labradorite, less common, as well as amphibole, pyroxene, quartz and the accessories: Zircon, apatite, ilmenite, magnetite and some hydrous iron-ore.

Three amphiboles are recognized, viz. 1) common hornblende 2) chestnut-brown hornblende (in association with pyroxene) and 3) a soda-amphibole of the arfvedsonite-group.

77. Greyish-white two-mica-granite. Metamorphic Complex.

NE. Øvre Rypegletcher (i. e. Upper Ptarmigan glacier), Kejser Franz Joseph Fjord.

Wordie, I. M. \& W. F. Whittard (1930, p. 650, no. 103). 
The following constituents are found: Orthoclase, often with a central sericitized region, microcline with microperthitic structure, acid oligoclase, quartz, biotite occasionally showing a little chloritization — with magnetite and rutile -, muscovite, but not in such quantities as biotite, and the accessories: Apatite, ore and zircon.

78. Grey porphyry with phenocrystic quartz. Palaeozoic (?).

NE. Hesteskofjeld, Canning Land. Conglomerate-ball from Devonian deposits.

Noe-NygaARD, A. ((1936), 1937, p. 112, no. 1104 a).

The conglomerate-ball is thought by $\mathrm{N}-\mathrm{N}$ to be related to the palaeozoic magmatites of Canning Land.

The rock is a greyish, rather fresh looking porphyry with phenocrysts of quartz, quite dull, whitish felspars and chlorite-altered biotite.

79. Greyish-green porphyry without phenocrystic quartz. Palaeozoic (?).

NE. Mt. Kollen, Canning Land. Conglomerate-ball from Lower Permian deposits.

Noe-NygaARD, A. ((1936), 1937, p. 112, no. 342 d).

The conglomerate-ball is thought by $\mathrm{N}-\mathrm{N}$ to be related to the palaeozoic magmatites of Canning Land.

The rock is a rather fresh, greyish-green porphyry with whitish felspar-phenocrysts, intensely weathered.

80. Porphyric granite. Palaeozoic (?).

NE. South side of Porfyrfjeld, Canning Land.

NoE-NygaARD, A. ((1936), 1937, p. 49, no. 1114).

The rock is clearly porphyric, the phenocrysts forming 40 vol- $\%$, the microgranitic and rather calcite-filled groundmass 60 vol- $\%$. The phenocrystic minerals occur in the following proportions: Quartz $24,9 \%$, orthoclase $32,8 \%$, plagioclase (oligoclase/andesine) $31,5 \%$, biotite $9,2 \%$, accessories (apatite, ore and zircon) 1,6\%.

81. Spherulitic riebeckite-trachyte (perhaps Sølvsbergite). Tertiary. NE. Cape Parry, northeast corner of Traill Island. Pebble from the beach.

Tyrrell, G. W. (1932, p. 522, no. 95).

A norm, but no mineral description is given in the text.

82. Biotite-dacite. Age unknown, probably Devonian.

NE. Erratic from moraine at the eastern glacier, south coast of Segelsällskapets Fjord.

Rittmann, A. (1940, p. 18, no. 2). 
The texture is holocrystalline, porphyric. The fine grained groundmass consists of quartz, orthoclase, plagioclase, some biotite, ore and apatite. The following vol- $\%$ were obtained on the integration table: Andesine-phenocrysts 25,4\%, quartz-phenocrysts $0,4 \%$, biotite-phenocrysts $14,4 \%$, apatite-phenocrysts $0,3 \%$, magnetite (lumps) $0,2 \%$, groundmass $58,3 \%$, secondary muscovite in andesine about 1,0 \%.

83. Red porphyry with phenocrystic quartz. Devonian.

NE. North side of Porfyrfjeld, Canning Land. Dyke.

NoE-NygaARD, A. ((1936), 1937, p. 88, no. 246).

The texture and constituents of the groundmass not determinable owing to disintegration and calcitisation. Among the phenocrysts can be distinguished: Quartz, orthoclase, plagioclase - both intensely disintegrated -, biotite and the accessories: Apatite, sphene with leucoxene, pyrite and zircon.

84. Granulitic gneiss. Metamorphic Complex.

NE. Cape Hope, south end of Liverpool Land.

Sahama (Sahlstein), T. G. (1935, p. 37, no. 1110).

The mineral composition is: Quartz, plagioclase, potash-feldspar, biotite, garnet, pyroxene, amphibole, serpentine, chlorite, calcite and the accessories: Apatite, rutile, ore and zircon.

85. Anorthoclase-trachyte. Tertiary.

NW. Arfertuarssuk Fjord, Svartenhuk Peninsula.

Nieland, H. (1931, p. 598).

A light-grey rock (with a reddish weathering-hue), which owing to numerous big anorthoclase-phenocrysts has a coarse-grained appearance; single small phenocrysts of pyroxene and ore are also found.

The groundmass may be coarser — orthophyric, or finer more trachytic. The main constituent is anorthoclase. Some interstitial, now partly devitrified glass occurs, as well as quartz and titaniferous iron-ore. Of sparse occurrence there are hornblende, aegirine-augite, aegirine, apatite, limonite and goethite.

86. Astochite-gneiss. Metamorphic Complex. (Precambian).

NW. Right side of the Qarajaq glacier.

Belowsky, M. (1905, p. 37).

The gneiss is a light reddish-grey rock consisting of orthoclase, albite - with curved lamellae, blue hornblende (soda-richterite) and the accessories: Magnesia-mica, apatite, sphene and zircon. Quartz is totally wanting. The structure is crystalloblastic.

87. Syenite. Tertiary.

NE. The cape just to the west of the entrance to Antarctic Harbour, mouth of King Oscar Fjord.

NoE-NygaARD, A. (1940, p. 555 , no. 450$)$. 
A coarse to medium-grained syenite with a little quartz. The constituents are: Perthite ( 78 vol- $\%$ of the total felspars), anti-perthite $(20 \%)$, plagioclase (andesine) (2\%), cores of diopsidic pyroxene encircled by two generations of amphiboles, biotite and a little quartz interstitially. The accessories are: Sphene, apatite, zircon, rutile and ore. The vol- $\%$ of the constituents are the following: Felspars $85,8 \%$, quartz $1,5 \%$, pyroxene $0,2 \%$, amphiboles $6,5 \%$, biotite $1,0 \%$, and accessories $5,0 \%$.

88. Nepheline-sodalite-syenite. Tertiary.

SE. West side of Kangerdlugssuaq.

WAGER, L. R. (1934, p. 39).

A norm is given on p. 39 ; no description of the mineral content is given in the text.

89. Reddish-brown porphyry without phenocrystic quartz. Devonian.

NE. North side of Porfyrfjeld, Canning Land.

Noe-NygaARD, A. ((1936), 1937, p. 82, no. 248).

The groundmass is entirely altered and filled with calcite.

The following vol- $\%$ of the phenocrysts are recorded: Orthoclase + plagioclase $67,3 \%$, biotite $23,5 \%$, pseudomorphs on pyriboles $2,7 \%$ and accessories $6,5 \%$.

90. Greyish-green porphyry without phenocrystic quartz. Devonian. NE. Cape Fletcher, Canning Land.

NOE-NyGaARD, A. ((1936), 1937, p. 70, no. 363).

The groundmass is of microgranitic to microgranophyric texture, but very unfresh. The following vol- $\%$ of the phenocrysts are recorded: Orthoclase $36,0 \%$, biotite $9,8 \%$, pseudomorphs on orthorhombic pyroxene $22,1 \%$, plagioclase (andesine) $29,1 \%$, clinopyroxene \& pseudomorphs on clinopyroxene $3,1 \%$.

91. "Aplite" from the Kaersutite-pegmatite. Tertiary.

NW. Østerfjeld, Qaersut, Nûgssuaq Peninsula.

Drescher, F. K. \& H. K. E. Krueger (1928, p. 602).

The volumetric determination of the mineral content is the following: Anorthoclase $81,0 \%$, pyroxene and amphibole 5,0\%, biotite $3,5 \%$ and zeolites $10,5 \%$.

92. Iron-bearing rock. Tertiary.

NW. Ivigsarkut.

NicolaU, Th. (1901, p. 243, no. 474).

The rock is very similar to the rock of Jernpynten (no. 94), only that orthorhombic pyroxene is less common both as phenocrysts and in the groundmass. 
93. Reddish-brown porphyry without phenocrystic quartz. Devonian. NE. Western side of Cape Fletcher, Canning Land.

NOE-NyGAARD, A. ((1936), 1937, p. 82, no. 353).

The groundmass has a microgranitic to microfelsitic texture. The following are the vol- $\%$ of the phenocrysts. Orthoclase $23,3 \%$, biotite $14,3 \%$, pseudomorphs on orthorhombic pyroxene $30,0 \%$, plagioclase (andesine) $9,3 \%$, pseudomorphs on clinopyroxene $15,1 \%$ and accessories $8,0 \%$.

94. Iron-bearing rock. Tertiary.

NW. Jernpynten, west side of Disko.

NicolaU, Th. (1901, p. 243, no. 506).

The texture is clearly porphyric. The groundmass consists of labradorite-microliths (in flow-arrangement) and a mixture of clinopyroxene and enstatite-laths. Ore and isotropic hisingerite are rare. Homogeneous areas are occupied by iron. Big phenocrysts consist of bytownite, often zoned, dotted with graphite dust and enstatite and more seldom clinopyroxene.

95. Hedenbergite-granophyre. Tertiary.

SE. About $800 \mathrm{~m}$ above sea-level, west face of Brødretoppen, Kangerdlugssuaq.

Wager, L. R. \& W. A. Deer (1939, p. 210, no. 3047).

The rock belongs to the group of »transgressive rocks of the Skaergaard Intrusion. The following mode is given: Quartz $\left.{ }^{1}\right) 36 \%$, clinopyroxene $13 \%$, plagioclase $40 \%$, ore ${ }^{2}$ ) $11 \%$.

96. Granodiorite. Metamorphic Complex. (Caledonian).

NE. Cape Smith, Liverpool Land.

Kranck, E. H. (1935, p. 74).

Same main-constituents in almost the same proportions as in granodiorite from Hodal, Liverpool Land (no. 106).

97. Nordmarkite. Age unknown, probably Precambrian.

SW. Narsak (the specimen analyzed was taken on the west side of Kakarsuak).

Ussing, N. V. (1911, p. 196, no. 17).

A coarse-grained greyish-red alkali-syenite, which U.M. shows the following composition: Soda-orthoclase with some oligoclase, green and brown hornblende, colourless or greyish augite, some biotite, iron-ore, apatite and finally a little hydrous iron-oxide arisen from the decomposition of the ferro-magnesian minerals.

1) Includes micropegmatite and dusty borders of plagioclases.

2) Includes some chlorite. 
98. Pulaskite. Age unknown, probably Precambrian.

SW. N. Siorarsuit, north side of Tunugdliarfik.

Ussing, N. V. (1911, p. 124, no. 3).

A white coarse-grained rock, consisting of rather thick, tabular felspars and dark minerals filling out the interspaces between them. The constituents are: Felspar (microperthitic microcline-albite), arfvedsonite, aegirine, aegirine-augite and biotite, as well as small quantities of magnetite and fluorite. Zeolites and probably catapleite occur as secondary products. The original presence of scarce grains of nepheline, enigmatite and eudialyte is indicated by pseudomorphs observed in some of the samples.

99. Granodiorite. Metamorphic Complex. (Caledonian). NE. Raffles Island off the coast of Liverpool Land.

Kranck, E. H. (1935, p. 67).

The constituents are: Plagioclase (18\% an) $38,61 \%$, quartz $12,61 \%$, microcline $18,0 \%$, dark green hornblende with remnants of augite cores $26,50 \%$, biotite $2,14 \%$, magnetite $2,14 \%$, and a little epidote and small quantities of apatite and sphene.

100. Acid quartz-gabbro. Tertiary.

SE. North face of Østtoppen, $450 \mathrm{~m}$ below summit Kangerdlugssuaq.

WAGER, L. R. \& W. A. Deer (1939, p. 170, no.4163).

Belongs to the upper boundary group of the Skaergaard Intrusion and has the following mode:

Quartz (mainly micropegmatite but includes also dusty borders of plagioclase) $21 \%$, clinopyroxene with $\gamma=1,732$ (about one tenth replaced by chlorite) $28 \%$, plagioclase (strongly zoned from andesine to albite) $42 \%$ and ore $9 \%$.

101. Hedrumite. Age unknown, probably Precambrian.

SW. Akuliarusek, Igaliko Fjord. $3 \mathrm{~m}$ wide dyke.

Ussing, N. V. (1911, p. 279, no. 29).

The rock is fine-grained, devoid of phenocrysts and of trachytoid texture. The main constituent is felspar (orthoclase and plagioclase in perthitic intergrowth). Between the felspars occur aggregates of muscovite, which is probably secondary and replacing nepheline, brownish-green hornblende, grey augite, aegirine and green biotite; apatite and iron-ore occur but sparingly.

102. Arfvedsonite-lujavrite. Age unknown, probably Precambrian.

SW. Nunasarnak, north side of Tunugdliarfik.

UsSING, N. V. (1911, p. 175, no. 12).

Very much like the arfvedsonite-lujavrite from Lille Elv (no. 116) but U. M. the Nunasarnak rock proves richer in arfvedsonite and poorer in aegirine. 
103. Foyaite. Age unknown, probably Precambrian.

SW. The mountain plateau above Naujakasik, south side of Tunugdliarfik.

USSING, N. V. (1911, p. 132, no. 4).

Uppermost sheet of nepheline-syenite complex, thickness $0-10 \mathrm{~m}$. U.M. the following main constituents are seen: Felspar (microperthitic microcline and albite), nepheline, aegirine-augite and catophorite-like hornblende. Subordinate constituents, sometimes wanting, are: Sodalite, biotite, aegirine, arfvedsonite, ainigmatite, olivine and eudialyte. Apatite and iron-ore are always present. Catapleite, cancrinite, hydronepheline (»spreustein«) and analcite occur as secondary constituents.

104. Augite-syenite. Age unknown, probably Precambrian.

SW. Nunasarnausak, north coast of Kangerdluarsuk.

Ussing, N. V. (1911, p. 190, no. 16).

A coarse to medium-grained greyish rock in which the dark minerals form conspicuous spots، No parallel arrangement of minerals perceptible; the felspars occur in irregular grains. U. M. the main constituents are: Soda-orthoclase, augite and olivine. In smaller quantities there are: Nepheline, green hornblende, barkevikite, biotite, iron-ore and apatite.

105. Cf. Leuko-syenite.

SW. Ivigtut Peninsula.

(Unpublished analysis, submitted to us by R. BøGVAD).

No rock-description.

106. Granodiorite. Metamorphic Complex. (Caledonian).

NE. Hodal north of Hurry Inlet, western side of Liverpool Land. Kranck, E. H. (1935, p. 73).

The following vol- $\%$ are given: Quartz $6,75 \%$, plagioclase $(27 \%$ an) $48,39 \%$, potash-felspar $7,24 \%$, hornblende $22,60 \%$, augite $4,62 \%$ and biotite $10,40 \%$. The accessories are: Ore, sphene and apatite.

107. Hedenbergite-andesinite. Tertiary.

SE. West face of Basistoppen, $250 \mathrm{~m}$ above "purble band", Kangerdlugssuaq.

WAgER, L. R. \& W. A. DeER (1939, p. 114, no. 4136).

Belongs to the unlaminated, layered series of the Skaergaard Intrusion. Following vol- $\%$ are given: Quartz $\left.^{1}\right) 26 \%$, plagioclase $56 \%$, clinopyroxene (in composition close to hedenbergite) $15 \%$, ore $2 \%$ and apatite $1 \%$.

1) This figure is for material which is interstitial to the early well-shaped plagioclase; it includes an outer border of felspar in graphic intergrowth with quartz and also clear quartz and some apatite and chlorite. 
108. Iron-bearing dolerite. Tertiary.

NW. Asuk, north coast of Disko Island.

SMITH, L. (1879, p. 486).

The iron particles are more rounded than those in the Uivfaq dolerite and are encircled by crystals of plagioclase (undoubtedly labradorite, perhaps with some oligoclase). The microlithes of plagioclase have a fluidal arrangement round the big crystals of augite. Nicolau (Medd. om Grønland Vol. 24 p. 241) feels inclined to term this rock an andesite (perhaps hypersthene-andesite) because of the high silica content.

109. Nepheline-porphyry. Age unknown, probably Precambrian.

SW. Akuliarusek on the peninsula south of Fox Bay, Igaliko Fjord. A $13 \mathrm{~m}$ wide composite dyke (central part of dyke).

Ussing, N. V. (1911, p. 275, no. 28).

Phenocrysts are: Felspars (alkali-felspars), originally nepheline, (now altered into psedumorps consisting of muscovite with felspar) an unknown colourless mineral and original big crystals of greyish augite altered partly into fibrous green hornblende. The groundmass consists of microperthite, nepheline, aegirine, green biotite, grey augite and often a little analcite.

110. Aegirine-lujavrite. Age unknown, probably Precambrian.

SW. Laxefjeld, Kangerdluarsuk.

Ussing, N. V. (1911, p. 168, no. 8).

A dark-coloured, fine-grained rock underlying naujaite; a conspicuous parallel arrangement of the minerals produces a marked schistosity. The constituents are: Felspar (microcline and albite), nepheline, eudialyte, aegirine and arfvedsonite. Minor accessories often entirely absent: fluorite, sphalerite, biotite and astrophyllite being present only in few instances. Secondary minerals: Analcite, catapleite, hydronephelite and natrolite. (In the sample analyzed the only felspar-component is microcline, analcite is scarce and hydronephelite largely replaces the nepheline).

111. Augite-syenite. Age unknown, probably Precambrian.

SW. Niakornarsuk on the northwestern side of Korok Fjord.

UsSing, N. V. (1911, p. 243, no. 26).

A coarse-grained dark-grey rock with the following constituents: Felspar, violet augite, some olivine and iron-ore and very small quantities of brown hornblende, biotite and apatite. ${ }^{1}$.

112. Normalfoyaite.

SW. Ivigtut Peninsula.

(Unpublished analysis, submitted to us by R. BøGVAD).

No rock-description.

\footnotetext{
1) As most of the varieties of the Igaliko syenite differ from the specimen analyzed in not containing olivine, it is probable that the general composition of the rock is a little more acid than that of the variety analyzed.
} 
113. Foyaite. Age unknown, probably Precambrian. SW. NNW. of Igdlerisalik on the south coast of Korok Fjord. Ussing, N. V. (1911, p. 235, no. 25).

A coarse-grained, reddish-grey rock with the following constituents: Felspar (perthites, the potash-felspar commonly being orthoclase, the plagioclase being an acid oligoclase), nepheline, cancrinite, iron-ore, aegirine-augite, hornblende, biotite, apatite and various alteration products (zeolites and calcite). Small amounts of zircon, fluorite (found only in one sample) and a rinkite-like mineral also occur. (In the sample analyzed aegirine-augite predominates among the dark constituents; sodalite and nepheline occur only in very small quantities).

114. Aegirine-lujavrite. Age unknown, probably Precambrian.

SW. Tupersuatsiak, south side af Tunugdliarfik Fjord.

Ussing, N. V. (1911, p. 168, no. 9).

The rock analyzed differs from no. 110 in the proportion of felspars, albite being the dominant one; microcline is present only subordinately. Arfvedsonite is rather scarce and is partly converted into acmite. Analcite is almost absent, but the outer zone of the nephelinecrystals has been transformed into sodalite; the interior has partly changed into hydronephelite.

115. Eklogite. Metamorphic Complex.

NE. Hurry Inlet side of Liverpool Land.

Sahama, Th. G. (1935, p. 11).

The rock is fresh and not altered; the volumetric analysis of the rock shows the following composition: Pyroxene 66,2\%, garnet $24,4 \%$, amphibole $1,3 \%$, quartz $7,6 \%$, rutile $0,5 \%$. A little ore also occurs.

116. Arfvedsonite-lujavrite. Age unknown, probably Precambrian.

SW. Lille Elv, Kangerdluarsuk.

Ussing, N. V. (1911, p. 175, no. 11).

Dark, greyish, moderately fine-grained with somewhat schistose structure. U. M. the following components are seen: Arfvedsonite, aegirine, albite, microcline, nepheline, sodalite and minor accessories. Albite is more abundant than microcline, and aegirine is common. Analcite and hydronephelite occur only in small quantities.

117. Iron-dolerite. Tertiary.

NW. Inner part of Mellemfjord, western side of Disko.

LORENTZEN, J. (1883, p. 146).

Dolerite of medium grain and containing only large pieces of iron. The iron was consequently easily isolated from the host-rock. 
118. Altered nepheline-basalt. Tertiary.

SE. West side of Kangerdlugssuaq. Erratic.

WAGER, L. R. (1934, p. 32).

A norm is given on p. 32, no description of the actual mineral content in the text. (The rock considered to be a lava, not belonging to a dyke).

119. Tremolite-schist. Metamorphic Complex (Precambrian). NW. Uvkusigssat in Lille Qarajaq Fjord.

Belowsky, M. (1905, p. 45).

The rock consists exclusively of tremolite and a light magnesiamica. (It can be traced genetically back to peridotitic rocks).

120. Lamprophyre (kersantitic). Palaeozoic.

NE. Porfyrfjeld, Canning Land. Dyke.

NoE-NygaARD, A. ((1936), 1937 p. 103, no. 1110).

The groundmass is pilotaxitic to hyalopilitic and filled with calcite. Biotite is the dominant and only unaltered phenocryst. Accessories are: Apatite, epidote, hematite, rutile, zircon and black ore. Secondary are calcite, chlorite, sericite and quartz. In amygdules barite.

121. Basic hedenbergite-granophyre. Tertiary.

SE. West face of Basistoppen, $220 \mathrm{~m}$ above "purple band", Kangerdlugssuaq.

Wager, L. R. \& W. A. Deer (1939, p. 114, no. 4137).

Belongs to the unlaminated, layered series of the Skaergaard Intrusion and has the mode: Quartz ${ }^{1}$ ) $39 \%$, clinopyroxene ${ }^{2}$ ) $22 \%$ (close to hedenbergite in composition), plagioclase $28 \%$ (centre $a_{40}$ outer half $\mathrm{an}_{25}$ ), ore $10 \%$, apatite $1 \%$.

122. Kakortokite (white sheets). Age unknown, probably Precambrian. SW. Kringlerne, south side of Kangerdluarsuk Fjord.

Ussing, N. V. (1911, p. 182, no. 13).

The rock is differentiated in sheets of various colours and compositions (vide no.s 131 and 136). Maximal thickness about $400 \mathrm{~m}$. The kakortokite is over and underlain by lujavrite. The mineral composition is: Eudialyte, alkali-felspar, nepheline, arfvedsonite and aegirine. Occasional accessories: Sodalite, enigmatite, biotite, rinkite, fluorite and rarely epistolite. Zeolites occur as secondary products.

1) This figure is for material which is interstitial to early well-shaped plagioclase; it includes an outer border of felspar in graphic intergrowth with quartz, clear quartz and also some apatite and chlorite.

2) Includes a little chlorite. 
123. Nepheline-porphyry. Age unknown, probably Precambrian. SW. Akuliarusek, on the northern side af Fox Bay, Igaliko Fjord. Ussing, N. V. (1911, p. 275, no. 27).

A porphyry with large phenocrysts of nepheline and felspar in a violet-brown, almost dense groundmass. Felspar less numerous than nepheline; the felspar is soda-orthoclase, sometimes with a little albite. Augite-phenocrysts were probably present originally. The groundmass consists of: Felspar, nepheline, sodalite, analcite, cancrinite, fluorite, green biotite, aegirine, garnet (?), and iron-ore. Apatite is rare.

124. Hornblende-vogesite. Post-Cambrian.

NE. Bastionen, Ella Island.

RitTmann, A. (1940, p. 147).

The groundmass consists of albite in hypautomorphic crystals, allotriomorphic orthoclase and some quartz. Accessories are: Ore, apatite and epidote. Secondary penninite is found in pyroxene and amphibole. The vol- $\%$ are: Hornblende $48 \%$, alkali-felspars $37 \%$, diopsidic augite $6 \%$, quartz $3 \%$, magnetite $2 \%$, epidote $1 \%$, apatite $1 \%$ and penninite (secondary) $2 \%$.

125. Essexite-porphyrite. Age unknown, probably Precambrian.

SW. Kakarsuak, upper part of the mountain, Narsak. Minor instrusive body of irregular shape.

Ussing, N. V. (1911, p. 208, no. 20).

A porphyritic rock with numerous and large plagioclase-phenocrysts in a fine-grained, greenish-black groundmass. The felsparphenocrysts consist of labradorite, the smaller ones often having a coating of orthoclase. The groundmass consists of: Plagioclase, orthoclase, augite, iron-ore and apatite. The rock is greatly altered by contact-metamorphism, and the original texture of the groundmass is quite effaced.

126. Aegirine-lujavrite. Age unknown, probably Precambrian. SW. S. Siorarsuit, south side af Tunugdliarfik Fjord.

Ussing, N. V. (1911, p. 168, no. 10).

This rock is in an advanced state of zeolitization. The aegirine is intact, but the great majority of the light coloured minerals have been replaced by analcite.

126 a. Arfvedsonite-leucite-tinguaite. Age unknown, probably Precambrian.

SW. Kangerdluarsuk.

Rosenbusch, H. (1898, p. 215).

Leucite inverted to analcite. Considerable content of eudialyte. 
127. Iron-dolerite. Tertiary.

SW. Fiskernæsset, Godthaab district. Erratic.

LoREnTZEN, J. (1883, p. 155).

The microscopical examination by K. I. V. Steenstrup. The finegrained groundmass consists of small crystals of a triclinic felspar surrounded by rounded grains of augite, olivine and branchy patches of magnetite or ilmenite. The average size is $0,3 \mathrm{~mm}$. Larger crystals of triclinic felspar up to $1,5 \mathrm{~mm}$ long are met with scattered in the groundmass. The minerals are fresh.

128. Olivine-gabbro. Tertiary.

SE. $200 \mathrm{~m}$ from outer contact, foot of west ridge of Tinden, Kangerdlugssuaq.

WAger, L. R. \& W. A. Deer (1939, p. 167, no. 2275).

The rock belongs to the marginal border group of the Skaergaard Intrusion and has the following modal composition: Quartz $6 \%$, olivine $4 \%(\gamma=1,775)$, plagioclase $53 \%$ (deeply zoned, $\left.a_{75}\right)$, clinopyroxene $\left.^{1}\right) 27 \%(\gamma=1,718)$, ore $10 \%$.

129. Ilimausak-porphyry. Age unknown, probably Precambrian.

SW. Ilimausak at a height of about $800 \mathrm{~m}$, at North Siorarsuit in the talus at the foot of Mt. Hatten and comes from the upper part of this mountain(?). Probably effusive sheets.

Ussing, N. V. (1911, p. 220, no. 23).

The rock is intensely altered by contact-metamorphism. Phenocrysts of felspar are anorthoclases with a composition ranging from that of an almost pure potash-felspar to that of albite; apatite and iron-ore also occur as phenocrysts. The fine-grained groundmass shows a marked flow-structure and consists mainly of small felsparlaths (alkali-felspar, probably anorthoclase), a great quantity of colourless needles, grains of titanite, scales of muscovite and minute grains of iron-ore.

130. Naujaite. Age unknown, probably Precambrian.

SW. Kangerdluarsuk.

Ussing, N. V. (1911, p. 154, no. 6).

The extremely coarse-grained rock of naujaite rests on lujavrite and is overlain by sodalite-foyaite. The main constituents are sodalite, nepheline, alkali-felspar (microcline-microperthite), aegirine, arfvedsonite, eudialyte and the minor accessories: Enigmatite, rinkite, rosenbuschite, molybdenite, sphalerite, biotite, polylithionite and apatite. Secondary products are: Analcite and natrolite.

1) Includes some chlorite. 
131. Kakortokite (red sheets). Age unknown, probably Precambrian. SW. Kringlerne, south of Kangerdluarsuk Fjord.

Ussing, N. V. (1911, p. 182, no. 14).

The same qualitative mineral composition as in the white kakortokite, (no.122) but eudialyte forms about one third of the rock.

132. Sodalite-foyaite. Age unknown, probably Precambrian.

SW. Tupersuatsiak, south side of Tunugdliarfik Fjord.

Ussing, N. V. (1911, p. 141, no. 5).

The coarse-grained rock occurs as a heavy sheet resting upon the naujaite and covered by foyaite. The components are: Alkali-felspar (microcline-microperthite), nepheline, sodalite, arfvedsonite, aegirine, enigmatite and locally also eudialyte. Minor accessories are: Magnetite, biotite, aegirine-augite, astrophyllite, rinkite, steenstrupine, polylithionite (?) and fluorite. Secondary products are: Catapleite, analcite and hydronephelite.

133. Dolerite. Tertiary.

NW. Inner part of Brededal, eastern slope of Skarvefjeld, Disko. NAUckHoff, E. G. R. (1872, p. 16, no. 1).

A dense, greenish-grey groundmass with cryptocrystalline texture in which U. M. the single individuals of felspar, augite and iron-ore can be seen. The felspars may attain a size of $0,5-1,0 \mathrm{~mm}$ in length. Olivine is wanting.

134. Quartz-gabbro. Tertiary.

SE. $200 \mathrm{~m}$ below summit of Brødretoppen, Kangerdlugssuaq.

Wager, L. R. \& W. A. Deer (1939, p. 170, no. 3050).

The rock belongs to the upper border group of the Skaergaard Intrusion, and has the following mode: Plagioclase 57,0\% $\left(\mathrm{an}_{56}\right)$, clinopyroxene $37,0 \%(\gamma=1,719)$, quartz $3,0 \%$ and ore $3,0 \%$.

135. Dolerite. Tertiary.

NW. Uivfaq, Disko.

Sмiтh, L. (1879, p. 474 II).

Dolerite, not iron-bearing.

136. Kakortokite (black sheets). Age unknown, probably Precambrian. SW. Kringlerne, south side of Kangerdluarsuk Fjord.

Ussing, N. V. (1911, p. 182, no. 15).

Same qualitative mineral composition as no. 122. In spite of its extremely melanocratic character the rock is entirely devoid of iron-ore. 
137. Tholeitic basalt. Tertiary.

SE. Cape Dalton, Blosseville Coast.

WAGER, L. R. (1934, p. 32).

No mineral description in text.

138. Eklogite (partly converted into amphibolite). Metamorphic Complex.

NE. Hurry Inlet side of Liverpool Land.

Sahama, Th. G. (1935, p. 13).

Cf. no. 178. The rock has been partly amphibolitized.

139. Pigeonite-tholeite. Piercing Devonian strata.

NE. North coast of the outer Geolog Fjord Andrée Land. Dyke. Rittmann, A. (1940, p. 109, no. 34).

Texture doleritic with intersertal granophyre and chlorite. Idiomorphic to hypidiomorphic pyroxene, ilmenite and magnetite crystals are found between the larger plagioclase laths and cause their allotriomorphic outlines. The vol- $\%$ are the following: Plagioclase $(50 \%$ an $45,4 \%$, pigeonite $18,5 \%$, ilmenite and hematite $8,2 \%$ and fillingmass $27,9 \%$. The filling-mass consists of about two thirds of alkalifelspars and quartz and about one third chlorite and a little apatite and magnetite.

140. Dolerite. Tertiary.

NE. Jackson Island, Gael Hamke Bay.

Made for Dr. Tyrrell, G. W., published by Backlund, H. G., \& D. MALmQuist (1932, p. 46).

No mineral description in text, but by authors compared with no. 145 .

141. Kaersutite pegmatite. Tertiary.

NW. Østerfjeld, Qaersut, Nûgssuaq Peninsula.

Drescher, F. K. \& H. K. E. Krueger (1928, p. 598).

Main constituents are: Kaersutite-hornblende, plagioclase (55$58 \%$ an), potash-felspars and zeolites. Subordinate constituents are: Biotite, augite and ilmenite. The only accessory mineral is apatite. Secondary products are kaolinite, nontronite (locally) and calcite.

142. Hornblende-biotite-sehist. Metamorphic Complex.

NE. Opposite to Riddarborgen. Franz Joseph Fjord.

Wiseman, I. D. H. (1931, p. 320, no. 128).

Prior to metamorphism this schist was of a basic igneous type. In the field an even-grained, dark-green amphibolite. U. M. largely idioblastic hornblende crystals, quartz and rutile found as inclusions, biotite in small amounts. A little quartz is met with interstitially between the hornblende crystals. 
143. Fayalite-ferrogabbro. Tertiary.

SE. $10 \mathrm{~m}$ from top of more easterly nunatak to the south of Forbindelsesgletcher, Kangerdlugssuaq.

Wager, L. R. \& W. A. Deer (1939, p. 106, no. 1881).

The rock belongs to the layered series of the Skaergaard Intrusion, and has the following mode: Quartz $11 \%,{ }^{1}$ ), clinopyroxene $34 \%$, plagioclase $29 \%$, ore $8 \%$, olivine $\left(\mathrm{Fa}_{96}\right) 17 \%$ and apatite $1 \%$.

144. Iron-bearing dolerite. Tertiary.

NW. Uivfaq, Disko.

Smith, L. (1879, p. 475, no. 15).

The rock contains augite, plagioclase and a considerable amount of olivine in big crystals of irregular and often corroded forms, as well as black iron-ore. Interstitially a vitreous residue is found impregnated with iron-ore and filled with alteration material. The metallic iron occurs as angular grains.

145. Dolerite. Tertiary.

NE. Kirchenpauer Bay, Clavering Island.

Backlund, H. G. \& D. Malmeuist (1932, p. 24).

The vol- $\%$ of the constituents are the following: Plagioclase (55\% an) $39,3 \%$, anorthoclase and microcline $8,6 \%$, pyroxene $32,3 \%$, iddingsite $0,8 \%$, ore $10,5 \%$, quartz $1,3 \%$, apatite $0,7 \%$ and porosity $6,5 \%$.

146. Middle gabbro. Tertiary.

NE. $25 \mathrm{~m}$ above sea-level, west foot of Pukugaqryggen. Kangerdlugssuaq.

WAger, L. R. \& W. A. DeER (1939, p. 96, no. 3662).

The rock belongs to the layered series of the Skaergaard Intrusion, and has the following mode: Plagioclase $60 \%\left(\mathrm{an}_{35}\right)$, clinopyroxene $\left.\mathrm{e}^{2}\right)$ $33 \%$, orthopyroxene $2 \%$, ore $5 \%$ and apatite tr.

147. Basalt, free from olivine. Tertiary.

NW. Hare Island.

Holmes, A. (1918, p. 183, no. 1554).

An almost black basalt with rusty amygdules (with chlorite, chalcedony and calcite). The texture is intergranular. The main mass of the rock consists of laths of labradorite between which occur granular aggregates of pale green augite together with a few slightly larger individuals of hypersthene. Scattered profusely among the other constituents are ragged patches of hematite and minute globules and irregular masses of magnetite and ilmenite. There is also a mineral with mottled extinction and a refringence lower than that of Canada balsam; it is certainly an alkali-felspar, probably anorthoclase.

$\left.{ }^{1}\right)$ Includes marginal felspar, probably perthitic

$\left.{ }^{2}\right)$ Analyzed separately (Wo 32,3 , En 32,1, Fs 35,6 weight-\%) op. cit. p. 77 . 
148. Iron-bearing basalt. Tertiary.

NW. The eastern part of the iron-bearing dyke at Uivfaq, Disko. Nauckhoff, E. G. R. (1872, p. 17, no. 2).

A dark-brown rock of fine grained texture, consisting of labradorite, greenish-brown augite and magnetic iron-ore. From the margin of the dyke there is also mention of a greenish hisingeritelike mineral and yellowish-brown sulphuric iron. No metallic iron present. The rock melts rather easily to the blowpipe.

149. Quartz-gabbro. Tertiary.

SE. Summit of Brødretoppen, Kangerdlugssuaq.

WAGER, L. R. \& W. A. DeER (1939, p. 170-71, no. 3052).

The rock belongs to the upper border group of the Skaergaard Intrusion (cf. no.: 134). Mode: Quartz ${ }^{1}$ ) 21,9\%, plagioclase 55,5\%, clinopyroxene $\left.{ }^{2}\right) 20,6 \%$, ore $2 \%$.

150. Dolerite. Tertiary.

NW. A little distance from the iron locality at Uivfaq, Disko.

Sмiтн, L. (1879, p. 474, no. I).

No metallic iron in this rock. No mineral description in the text.

151. Olivine-gabbro, marginal. Tertiary.

SE. $3 \mathrm{~m}$ from contact, head of Udløberen, Kangerdlugssuaq.

Wager, L. R. \& W. A. Deer (1939, p. 140, no. 1825).

The rock belongs to the chilled marginal gabbro group of the Skaergaard Intrusion. (cf. no. 154). Mode: Plagioclase 65,5\%, clinopyroxene $21,2 \%$, orthopyroxene $\left.{ }^{3}\right) 1,7 \%$, olivine $11,1 \%$ and ore $0,5 \%$.

152. Iron-bearing dolerite. Tertiary.

NW. Rolled boulder on the shore, Uivfaq, Disko.

Sмith, L. (1879, p. 471, no. III).

Its appearance is very much like common basalt; but small particles of metallic iron, pyrite, graphite and a black, vitreous mineral, hisingerite, is to be seen megascopically. U. M. corundum and spinel are also seen in association with the graphite.

153. Altered variolitic basalt. Tertiary.

SE. Middle of $18 \mathrm{ft}$. flow, south point of Hængefjeld, Kangerdlugssuaq.

Wager, L. R. \& W. A. Deer (1939, p. 16, no. 383).

1) Micropegmatite with chlorite, apatite and quartz.

2) About $1 / 10$ replaced by chlorite.

$\left.{ }^{3}\right)$ Perhaps a little low, as doubtful material was assumed to be clinopyroxene. 
The rock is about »a mean between the average composition of plateau basalt and the non-porphyritic central basalt of the authors of the Mull Memoir. The felspar of the rock is, however, between oligoclase and albite, more exact determinations from refractive indices being hindered by the presence of inclusions of chlorite.

154. Marginal Olivine-gabbro. Tertiary.

SE. $25 \mathrm{~m}$ from contact, east side of Skærgaardsbugt, Kangerdlugssuaq.

WAger, L. R. \& W. A. Deer (1939, p. 140, no. 1724).

The rock belongs to the chilled marginal gabbro group of the Skaergaard Intrusion (cf. no. 151) Mode: Plagioclase $57,2 \%$, clinopyroxene $\left.^{1}\right) 25,5 \%$, olivine $\left.{ }^{2}\right) \quad 16,3 \%$ and ore $1,0 \%$.

155. Olivine-basalt. Tertiary.

SE. Cape Daussy.

WAGER, L. R. (1934, p. 32).

Related to olivine-basalt from the north side of Barclay Bay (no. 159). No description of mineral contents in the text.

156. "Diorite" (Amphibolite). Metamorphic complex (Precambrian). NW. Lower, dark band in Umanak mountain.

Phalen, W. C. $(1904$, p. 190 , no. 75,481$)$.

It has a decidedly laminated structure, produced by similar orientation of its two essential components: Amphibole and felspar.

Main constituents: Hornblende and labradorite.

Accessories: Magnetite, pyrite, hematite, zircon, apatite, garnet (in order of abundance). The amphibole is perfectly fresh and occurs in elongated, lath-shaped forms with glistening cleavage planes.

157. Trachydolerite (Tasek-porphyrite). Age unknown, probably Precambrian.

SW. Tasek, southwest end of the lake, about $4 \mathrm{~km}$ northeast of Narsak; a $750 \mathrm{~m}$ high mountain.

Ussing, N. V. (1911, p. 216, no. 22).

Probably effusive, and highly affected by contact metamorphism. The felspar lies in composition between an acid andesine and an almost pure albite. The dense, trachytoid groundmass is composed of: Felspar laths, green biotite, apatite and iron-ore.

158. Altered olivine-basalt. Tertiary.

SE. Extreme end of Mikis Fjord on the south side, near Kangerdlugssuaq.

Wager, L. R. \& W. A. Deer (1939, p. 16, no. 1519).

1) Includes some uralite.

2) Includes some serpentine. 
The rock corresponds to an olivine-rich basalt, but the minerals present are augite, oligoclase or albite, chlorite and zeolites. Some of the chlorite replaces former olivine crystals, but much has an interstitial arrangement.

159. Olivine-basalt. Tertiary.

SE. North side of Barclay Bay.

WAGER, L. R. (1934, p. 32).

No description of mineral contents in the text.

160. Iron-bearing dolerite. Tertiary.

NW. Uivfaq, Disko.

Nicolad, Th. (1901, p. 232).

Main constituents: Idiomorphic plagioclase $\left(\mathrm{an}_{7_{0}}\right)$, allotriomorphic pyroxene (basaltic augite). Accessories: Ilmenite, pyrrhotite. Irregularly distributed: Hisingerite with its fibrous alteration-products, graphite, metallic iron and a groundmass. The texture is ophitic.

161. Prowersite. Age unknown, probably Palaeozoic.

NE. South coast of Segelsällskapets Fjord.

Rittmann, A. (1940, p. 151, no. 63).

A fine-grained, dark grey rock with a rusty-yellow weathering colour. The groundmass is hypidiomorphic, consisting of orthoclase and albite, and in it are small crystals of reddish-brown biotite, colourless augite and some magnetite and apatite. Secondary calcite is present in small quantities.

Mode: Alkali-felspar $48 \%$, biotite $24 \%$, augite $22 \%$ and apatite, ore $(+$ calcite $) 6 \%$.

162. Olivine-gabbro. Tertiary.

SE. $30 \mathrm{~m}$ from contact, Mellemø, Skærgaarden, Kangerdlugssuaq.

Wager, L. R. \& W. A. Deer (1939, p. 156, no. 1837).

The rock belongs to the marginal border group of the Skaergaard Intrusion.

Mode: Plagioclase $64 \%$, clinopyroxene $\left.{ }^{1}\right) 8 \%$, orthopyroxene $2 \%$, olivine $\left.{ }^{2}\right) 25 \%$ and ore $1 \%$.

163. Amygdaloidal basalt (lava). Tertiary.

SE. South side af Scoresby Sund.

Holmes, A. (1918, p. 189).

U.M. the rock consists of: Glomeroporphyritic bytownite $\left(\mathrm{an}_{80}\right)$ in an insertal groundmass of small felspar laths, grains of green augite and colourless enstatite, and very numerous small crystals of magnetite and ilmenite. Here and there are flakes of bright red hematite. The felspars of the groundmass are much more sodic than the phenocrysts, being labradorite near to andesine.

$\left.{ }^{1}\right)$ Includes a little chlorite.

$\left.{ }^{2}\right)$ Includes a little talc. 
164. "Sonnenbrennerbasalt". Tertiary.

NW. The basal layer of the basalt formation, Godhavn, Disko.

Krueger, H. K. E. (1927, p. 275).

Columnar, fissured basalt with light spots on a dark ground. U. M.: In a fine-grained groundmass are 8-10\% plagioclase-phenocrysts $\left(\mathrm{an}_{68}\right)$. The groundmass consists of numerous small augite grains, elongated felspar-laths, much ore (probably titaniferous magnetite), and in small quantities: A dark glass and chloritic masses.

165. Amphibolite. Metamorphic Complex.

NE. Cape Swainson, Liverpool Land.

Kranck, E. H. (1935, p. 27).

Dark green hornblende, plagioclase $\left(\mathrm{an}_{36}\right)$, dark-brown biotite, muscovite, sphene and apatite. In the undeformed sections the rock contains rounded, fairly big grains of garnet.

166. Basalt. Tertiary.

NW. Lyngmarksfjeld at Godhavn, Disko.

Holler, K. (1933, p. 48).

No mineral description in the text.

167. Plagioclase-basalt. Tertiary.

NE. West of Cape Franklin. Sheet.

Backlund, H. G. \& D. MalmQuist (1932, p. 32).

Glomeroporphyritic parts are seen in a dense, sub-ophitic groundmass, mainly consisting of radiating plagioclase-needles and allotriomorphic pyroxene. The rock consists of: Plagioclase, ore, pyroxene, completely altered olivine and a residual mass.

168. Basalt. Tertiary.

NE. Neills Cliff, Jameson Land.

WOLFF, F. VON (1931, p. 933, no. 19).

No mineral description.

169. Iron-bearing dolerite. Tertiary.

NW. Uivfaq, Disko.

Smith, L. (1879, p. 471, no. II).

The rock contained metallic iron, hisingerite and sulphides, but dolerite fragments lacking these minerals could easily have been obtained.

170. Hypersthene-olivine gabbro. Tertiary

SE. Junction of Uttentals Plateau and WNW ridge of peak 1300 metres of Gabbrofjeld, Kangerdlugssuaq.

Wager, L. R. \& W. A. Deer (1939, p. 92, no. 4077). 
The rock belongs to the layered series of the Skarrgaard Intrusion.

Mode: Plagioclase 55,0\%, clinopyroxene 21,0\%, olivine $17,5 \%$, orthopyroxene $5,0 \%$, ore $1,5 \%$ and apatite trace.

171. Grønlandite (Hyperstene $\left.{ }^{1}\right)$-hornblendite). Metamorphic Complex (Precambrian?).

NW. Upernivik Island.

Machatschki, F. (1927, p. 173).

Vide: Grønlandite no. 173.

172. "Perpendicular" felspar rock. Tertiary.

SE. West side of Mellemø, Kangerdlugssuaq.

WAGER, L. R. \& W. A. DeER (1939, p. 146, no. 1851).

The rock belongs to the marginal border group of the Skaergaard Intrusion.

Mode: Plagioclase 54,2 \%, clinopyroxene $22,7 \%$, olivine $22,8 \%$ and ore $0,3 \%$.

173. Gronlandite (Hypersthene-hornblendite). Metamorphic Complex (Precambrian?).

NW. Upernivik Island.

Maснатschкi, F. (1927, p. 173) Compare no. 171.

The rock consists mainly of dark hornblende, $20 \%$ hypersthene and small quantities of olivine and ore.

174. Essexite. Age unknown, probably Precambrian.

SW. Panernak Bay, northwest of Narsak.

Ussing, N. V. (1911, p. 203, no. 18).

A coarse-grained, dark-grey rock with large tabular crystals of plagioclase. U. M.: Apatite, iron-ore, olivine, augite, hornblende, biotite, labradorite, orthoclase and secondary products. Nepheline has not been observed.

175. Basalt. Tertiary.

NW. Lyngmarksfjeld at Godhavn, Disko.

WolfF, F. von (1931, p. 932, no. 6).

No mineral description.

176. Basalt. Tertiary.

NW. Lyngmarksfjeld at Godhavn, Disko.

WolfF, F. von (1931, p. 932, no. 5).

No mineral description.

1) By mistake the grønlandite is characterized as an enstatite-hornblendite in the heading of the treatise. The mistake has been corrected by MACHATSCHKI himself in Zeitschr. für Kristallographie Bd. 71, 1929 (p. 219). 
177. Middle gabbro. Tertiary.

SE. $75 \mathrm{~m}$ above sea-level, west foot of Pugugaqryggen, Kangerdlugssuaq.

WAGer, L. R. \& W. A. Deer (1939, p. 96, no. 3661).

The rock belongs to the layered series of the Skaergaard Intrusion. Mode: Plagioclase $37 \%$, pyroxene $51 \%$, ore $12 \%$ and apatite trace.

178. Eklogite (strongly amphibolitized). Metamorphic Complex.

NE. Liverpool Land.

Sahama, Th. G. (1935, p. 14).

(cf. no. 138 and no. 115).

179. Olivine-free gabbro. Tertiary.

SE. $6 \mathrm{~m}$ from inner contact with the layered series. Southeast corner of Ivnarmiut, Kangerdlugssuaq.

WAger, L. R. \& W. A. Deer (1939, p. 156, no. 4298).

The rock belongs to the marginal border group of the Skaergaard Intrusion.

Mode: Plagioclase $56 \%$, pyroxenes $\left.{ }^{1}\right) 35 \%$, ore $9 \%$.

180. Hypersthene-olivine gabbro. Tertiary.

SE. $150 \mathrm{~m}$ from the northern margin. Uttentals Plateau, Kangerdlugssuaq.

WAger, L. R. \& W. A. DeEr. (1939, p. 92, no. 4087).

Transitional to the layered series of the Skaergaard Intrusion.

Mode: Plagioclase $56 \%$, clinopyroxene $29 \%$, olivine $11 \%$, orthopyroxene $3 \%$, ore $0,7 \%$ and apatite trace.

181. Trachydolerite. Age unknown, probably Precambrian.

SW. $560 \mathrm{~m}$ above sea-level on the north side of Nunasarnausak, near the lower boundary of the sheet, which is intrusive in the sandstone.

Ussing, N. V. (1911, p. 212, no. 21).

A dark, fine-grained rock with ophitic texture. U.M. the rock proves greatly altered. Apatite, iron-ore, olivine, augite, labradorite are original rock components. $\gg$ Orthoclase, perhaps nepheline, hornblende and mica have probably also been originally present, but the microscopical examination gives no downright proofs of this."

182. Fayalite-ferrogabbro. Tertiary.

SE. $100 \mathrm{~m}$ above the "purple band" west face of Basistoppen, Kangerdlugssuaq.

Wager, L. R. \& W. A. Deer (1939, p. 114-115, no. 4139).

$\left.{ }^{1}\right)$ Includes a little chlorite. 
The rock belongs to the unlaminated layered series of the Skaergaard Intrusion.

Mode: Quartz ${ }^{1}$ ) $9 \%$, plagioclase $26 \%$, clinopyroxene $33 \%$, olivine $18 \%$, ore $12 \%$ and apatite $2 \%$.

183. "Anortitfels". Tertiary.

NW. Uivfaq, Disko.

NAUckHofF, E. G. R. (1872, p. 27, no. 9).

The groundmass consists of felspar and of a green and brown mineral resembling augite. In some places red spinel is seen. Magnetic iron-ore and metallic iron are not observed.

184. Hortonolite-ferrogabbro. Tertiary.

SE. Basishusene, Kangerdlugssuaq.

Wager, L. R. \& W. A. Deer (1939, p. 102, no. 1907).

The rock belongs to the layered series of the Skaergaard Intrusion.

Mode: Plagioclase $56 \%$, clinopyroxene $20 \%$, olivine $16 \%$, ore $8 \%$ and apatite trace.

185. Ferrohortonolite-ferrogabbro. Tertiary.

SE. West ridge of Basistoppen, 250 above Basishusene, Kangerdlugssuaq.

Wager, R. L. \& W. A. Deer (1939, p. 106-107, no. 4145).

The rock belongs to the layered series of the Skaergaard Intrusion.

Mode: Quartz 1\%, plagioclase $45 \%$, clinopyroxene $28 \%$, olivine $17 \%$, ore $6,5 \%$ and apatite $2,5 \%$.

186. Dyke of nepheline-tephritic type (?). Age unknown, probably Palaeozoic.

NE. Cape Fletcher, Canning Land.

NORDENSKJÖLD, O. (1907, p. 207).

A grey, compact, hornstone-like rock with numerous porphyritic crystalline agglomerations of a light mica. U. M. biotite, yellowishgreen, fresh hornblende can be distinguished, and furthermore one or two light, much transformed minerals, the nature of which was difficult to determine. Nepheline was not observed with certainty.

187. Olivine basalt. Tertiary.

NW. Dyke about one day's journey from the head of Umivik Fjord, Svartenhuk.

NiEland, H. (1931, p. 601).

A dark grey basalt. The mineral contents: Lath-shaped bytownite $\left(\mathrm{an}_{84}\right)$, light brown augite with brown-violet edges of titaniferous augite, ore and as a sparse groundmass brown glass. The bytownite often has a cover of labradorite $\left(a_{55}\right)$. The olivine crystals (15-16\% fayalite) are larger than the other mineral components.

Mode: Bytownite $26,0 \%$, augite $47,0 \%$, olivine $10,5 \%$, ore $8 \%$, apatite $0,5 \%$ and glass $8,0 \%$.

1) Includes some ore. 
188. Olivine-trachybasalt. Tertiary.

NE. Mt. Nordhoek south side, Loch Fyne.

Backlund, H. \& D. MalmQuist (1932, p. 38).

Mode: Plagioclase $54,5 \%$, pyroxene $20,6 \%$, ore $6,5 \%$, olivine $17,0 \%$ and apatite $0,6 \%$, residue $0,8 \%$.

189. Fayalite-ferrogabbro (purple band). Tertiary.

SE. At $550 \mathrm{~m}$ west face of Basistoppen, Kangerdlugssuaq.

Wager, L. R. \& W. A. Deer (1939, p. 106-107, no. 4142).

The rock belongs to the layered series of the Skaergaard Intrusion.

Mode: Quartz ${ }^{1}$ ) $6 \%$, plagioclase $24 \%$, clinopyroxene $37 \%$, olivine $21 \%$, ore $9 \%$ and apatite $3 \%$.

190. Dolerite (sill in peridotite). Tertiary.

NW. Østerf jeld, Qaersut, Nûgssuaq.

Drescher, F. K. \& H. K. E. Krueger (1928, p. 589).

Ophitic texture. The mineral contents are as follows: Main constituents: Plagioclase, titaniferous augite, nontronitic masses with biotite. Minor constituents: Ilmenite, chlorite. Accessories: Apatite. Secondary: Calcite, zeolites.

191. Iron-bearing basalt. Tertiary.

NW. "Piece from surface of the largest meteorite", Uivfaq, Disko. NoRdEnSKJöld, A. E. (1870, p. 1066).

NoRdström, Th. (1871, p. 460).

No mineral description in the text.

192. Disintegrated "olivinestone". Metamorphic Complex. (Precambrian?).

SW. Siorarsuit at Kangamiut.

RøRDAM, K. (1884, p. 129).

Vide no. 208.

193. Albite-diabase. Precambrian $\left.{ }^{2}\right)$.

NE. Mt. Ramsay north foot, Gauss Peninsula.

BACKLUnd, H. G. (1932, p. 96, no. 1226).

An albite-diabase rich in potash and with intersertal structure. The mineral constituents are: Plagioclase (albite) and potash felspar, bluish-green chlorite, a little green hornblende, quartz, light green chlorite, calcite, apatite needles of considerable length and much ore.

$\left.{ }^{1}\right)$ Including some intergrowth of quartz and felspar.

2) According to BaCKLUND. 
194. Naujaite. Age unknown, probably Precambrian.

SW. Nunasarnak, at the north side of Tunugdliarfik.

UsSing, N. V. (1911, p. 154, no. 7).

Vide Naujaite, Kangerdluarsuk no. 130.

195. Ankaramite. Tertiary.

NE. Ladder Mountain, head of Musk Ox Fjord.

Backlund, H. G. (Tyrrel, G. W.) (1932, p. 46).

No mineral description in the text.

196. Iron-bearing basalt. Tertiary.

NW. Uivfaq, Disko.

NAuckhoff, E. G. R. (1872, p. 21, no. 5).

The rock has a $20-25 \mathrm{~mm}$ thick cover of a green, lamellated rock. (cf. no. 214). U. M.: A fine-grained mixture of felspar, sparse green augite (?), magnetic iron-ore, sparse olivine, sulphuric iron and metallic iron, often of a globular form $6-7 \mathrm{~mm}$ in diameter. The iron is usually surrounded by magnetite.

197. Peridotite var. Picrite. Tertiary.

NW. Qaersut, Nûgssuaq.

Phalen, W. C. $(1904$, p. 211, no. 75,488).

U. M. the following minerals were observed: Olivine, augite, chlorite, felspar, biotite, magnetite, limonite, hematite and apatite. Olivine is by far the most abundant constituent. It occurs in perfectly automorphic forms, excepting when corroded by the surrounding magma. The groundmass of the rock is formed by a violet-tinted augite, but faintly green in spots.

198. Olivine basalt. (Melanocratic olivine-trachydolerite or felspathic augitite). Tertiary.

NW. Hare Island.

Holmes, A. (1918, p. 187, no. 1553).

A dense, black rock free from vesicles. U. M.: Idiomorphic pyroxene, often in glomeroporphyritic groups. The augites frequently enclose a core of olivine, which in turn includes grains of iron-ore. Olivine is seen in small, corroded crystals, ilmenite is abundant, felspar (bytownite) is sparse. These minerals lie in a dense groundmass speckled with minute black grains and globulites.

199. Basalt. Tertiary.

NW. Lyngmarksfjeld at Godhavn, Disko.

WolfF, F. von (1931, p. 932, no. 8).

No mineral description in the text. 
200. Gabbro-picrite. Tertiary.

SE. Uttentals Plateau, $1 \mathrm{~km}$ north of Uttentals Sund, Kangerdlugssuaq.

WAger, L. R. \& W. A. DeER (1939, p. 162, no. 1682).

The rock belongs to the marginal border group of the Skaergaard Intrusion.

Mode: Plagioclase $15,8 \%$, clinopyroxene $13,8 \%$, hypersthene $4,7 \%$, olivine $65,0 \%$ and ore $\left.^{1}\right) \quad 0,7 \%$.

201. Basalt (fragments in the decomposed breccia). Tertiary.

NW. The basalt dyke at Uivfaq, Disko.

NAUckhoff, E. G. R. (1872, p. 33, no. 13).

The rock has the same aspect as the basalt forming the dyke (no. 148). Even the sulphuric iron has been identified. (The high sp. gr. and the considerable amounts of $\mathrm{Fe}_{2} \mathrm{O}_{3}$ indicate that all the particles of iron oxydes have not been removed).

202. Basalt. Tertiary.

NW. Lyngmarksf jeld at Godhavn, Disko.

WolfF, F. von (1931, p. 932, no. 9).

No mineral description in the text.

203. Felspar-bearing peridotite. Tertiary.

NW. Østerfjeld, Qaersut, Nûgssuaq.

Drescher, F. K. \& H. K. E. Krueger (1928, p. 582).

(Compare no. 209).

Major constituents: Olivine, pyroxene, chlorite, serpentine. Minor constituents: Titaniferous hornblende, labradorite $\left(\mathrm{an}_{50}\right)$, biotite, analcite. Accessories: Magnetite, apatite. Secondary constituents: Calcite, chalcedony, limonite.

204. Iron-bearing dolerite. Tertiary.

NW. Uivfaq, Disko.

SMith, L. (1879, p. 471, no. I).

The rock contains a considerable number of iron grains, which could not be completely separated, the result being a higher content of $\mathrm{FeO}$ than ought to be found in the rock.

205. Basalt. Tertiary.

NW. Lyngmarksfjeld at Godhavn, Disko. (Upper part of the series).

WOLFF, F. VON (1931, p. 932, no. 10).

No mineral description in the text.

${ }^{1}$ ) Excluding ore formed by decomposition of olivine. 
206. Soapstone. Metamorphic Complex (Precambrian?).

SW. Unortoq Fjord.

VRBA, K. (1874, p. 120).

The rock has a green yellowish-grey colour. U. M.: A fibrous, almost colourless mass with few apatite needles, much magnetite, and rectangular cavities, doubtless originating from outweathered felspar. The soapstone shows a gradual transition into the diorite porphyry on which it borders and is doubtless a transformation of the latter.

207. "Anortitfels". Tertiary.

NW. Uivfaq, Disko.

LORENZEN, J. (1883, p. 165).

Spinel, anorthite and graphite observed. The graphite content was burned away before the above analysis was undertaken and formed $6,78 \%$ of the rock. The rest $(93,22 \%)$, was analyzed and gave the above composition.

208. "Olivinestone". Metamorphic Complex (Precambrian?).

SW. Siorarsuit at Kangamiut.

RøвDAM, K. (1884, p. 129).

A fragile, yellowish-green rock with olivine, serpentine, a bottlegreen hornblende ( $\gg$ Grammatite $\ll$ ), magnesia-mica and chromite (comp. no. 192).

209. "Augitzüge", in peridotite. Tertiary.

NW. Østerfjeld, Qaersut, Nûgssuaq.

Drescher, F. K. \& H. K. E. Krueger (1928, p. 582).

The actual mineral composition is not given; the calculated minerals are (comp. no. 203) olivine 44,5\%, pyroxene $19,65 \%$, titaniferous hornblende $5,00 \%$, biotite $1,00 \%$, magnetite $2,80 \%$, labradorite $3,00 \%$, serpentine $7,00 \%$, analcite $1,63 \%$, calcite $0,90 \%$, chlorite (limonite) $13,10 \%=99,00 \%$.

210. "Anortitfels". Tertiary.

NW. Uivfaq, Disko.

Nauckhoff, E. G. R. (1872, p. 28, no. 10).

The groundmass is a mixture of felspar and a grey, scaly mineral, shining like graphite. Small, black spots occur sparsely in the felspar. Red spinel is of common occurrence. Sulphuric iron and metallic iron could not be identified.

211. "Mandelsteinkern". Tertiary.

NW. Lyngmarksfjeld at Godhavn, Disko.

Holler, K. (1933/34, p. 32).

An extremely weathered basalt, mainly consisting of more or less opaque iron oxydes, remains of augite, of pseudomorphosed plagioclase and much ore. In the felspar as well as in the cavities there are secondary products: Zeolites, calcite, chalcedony, opal and a little fibrous zeolite (mainly natrolite). 
212. "Anortitfels". Tertiary.

NW. The dyke at Uivfaq, Disko.

NAuckhoff, E. G. R. (1872, p. 26, no. 8).

A grey rock containing metallic iron. U. M. the rock consists of felspar with numerous grey, shining scales, and a black magnetite or graphite-like mineral, sparse green augite and in some places much red spinel.

213. Alnøite (Ouachitite). Age unknown, probably Palaeozoic.

NE. Liverpool Land, right opposite Fame Islands, a few km from the coast in a little valley. Dyke.

NORDENSKIÖLD, O. (1907, p. 212).

The groundmass is dark, basaltic with large phenocrysts (up to an inch) of augite and brown mica.

U. M. the groundmass consists of pyroxene, ore, apatite, calcite and a brownish almost isotropic mass (rich in nepheline?) $\gg \mathbf{A}$ few almost opaque crystals may possibly be perowskite

214. "Anortitfels" (cover round no. 196). Tertiary.

NW. Uivfaq, Disko.

NAUckHOFF, E. G. R. (1872, p. 24, no. 7).

The groundmass consists of felspar, presumably anorthite, often in large crystals, in which red spinel(?) occurs. Further, a green augitelike mineral is observed and scales of pure iron. No magnetite.

215. Magnesite-pyroxenite. Age unknown, probably Precambrian. SW. $2 \mathrm{~km}$ northwest of Narsak.

Ussing, N. V. (1911, p. 205, no. 19).

A fine-grained, black rock, which is strongly magnetic and sometimes possesses polarity. U. M.: Pyroxene, iron-ore (must be highly titaniferous), olivine and biotite.

216. Soapstone. Metamorphic Complex (Precambrian?).

SW. Locality unknown. Professor LAUBE acquired it in Lichtenau.

JANOvSKÝ, J. V. (1873, p. 1230) published the analysis.

VRBA, K., republished the analysis accompained by a mineral description (1874, p. 122).

A compact, lamellated aggregate of a dark green colour. U. M. a colourless, fibrous-granular, perfectly homogeneous mass, in which solitary crystals of magnetite or apatite are very occasionally observed. 
217. Soapstone. Metamorphic Complex (Precambrian?).

SW. Locality unknown. Professor LAUBE acquired it in Lichtenau.

JANOvskÝ, J. V. (1873, p. 1230).

Same specimen as no. 216.

218. "Bauxitähnliche Hülle". Tertiary.

NW. Lyngmarksfjeld at Godhavn, Disko.

Holler, K. (1933/34, p. 32).

Consists almost exclusively of more or less opaque masses. Colour dark reddish-brown; light-brown transparent remnants of augite and ore crystals are occasionally recognized, and only pseudomorphs of plagioclase are contained in it. The rock is completely hydrothermally altered. 
Analyses of Telluric Iron.

Appendi

\begin{tabular}{|c|c|c|c|c|c|c|c|c|c|c|c|c|}
\hline No. & Rock Name & Locality & District & Year & $\mathrm{Fe}$ & $\mathrm{Ni}$ & Co & $\mathrm{Cu}$ & $\mathrm{S}$ & $\mathrm{P}$ & $\mathrm{C}$ & $\mathrm{SiO}$ \\
\hline 219 & $\begin{array}{l}\text { Iron } \\
\text { (one of the big } \\
\text { blocks). }\end{array}$ & Uivfaq (shore), Disko & NW & 1870 & 84.49 & 2.48 & 0.07 & 0.27 & 1.52 & 0.20 & $\left.10.16^{1}\right)$ & $\operatorname{tr}$ \\
\hline 220 & $\begin{array}{l}\text { Iron } \\
\text { (one of the smal- } \\
\text { ler blocks). }\end{array}$ & Uivfaq, Disko & NW & 1870 & $\left.86.34^{1}\right)$ & 1.64 & 0.35 & 0.19 & $\left.0.22^{2}\right)$ & 0.07 & $\left.3.71^{3}\right)$ & 0.66 \\
\hline 221 & Iron & $\begin{array}{l}\text { The dyke at Uivfaq, } \\
\text { Disko }\end{array}$ & NW & 1870 & 93.24 & 1.24 & 0.56 & 0.19 & 1.21 & 0.03 & 2.30 & \\
\hline 222 & Iron & Uivfaq, Disko & NW & 1872 & $\left.88.67^{1}\right)$ & 2.16 & 0.30 & 0.13 & 0.16 & tr. & $\left.1.64^{2}\right)$ & 0.26 \\
\hline 223 & Breccia & $\begin{array}{l}\text { The dyke at Uivfaq, } \\
\text { Disko }\end{array}$ & NW & 1872 & $\left.80.27^{-1}\right)$ & 1.22 & 0.30 & 0.08 & 0.34 & 0.12 & 3.52 & 1.04 \\
\hline 224 & $\begin{array}{l}\text { Breccia } \\
\text { (weathered) }\end{array}$ & $\begin{array}{l}\text { The dyke at Uivfaq, } \\
\text { Disko }\end{array}$ & NW & 1872 & $\left.85.12^{1}\right)$ & 1.81 & 0.33 & $\left.0.30^{2}\right)$ & tr. & 0.12 & $\left.2.33^{3}\right)$ & 0.81 \\
\hline 225 & Iron & Uivfaq, Disko & NW & 1872 & 80.64 & 1.19 & 0.47 & tr. & 2.82 & 0.15 & 3.69 & $\left.{ }^{1}\right)$ \\
\hline 226 & $\begin{array}{l}\text { Iron } \\
\text { (Type I }\end{array}$ & Uivfaq, Disko & NW & 1872 & $\left.71.09^{1}\right)$ & 2.65 & 0.91 & $\left.1.01^{2}\right)$ & 2.70 & 0.21 & $\left.4.64^{3}\right)$ & $0.0^{\circ}$ \\
\hline 227 & $\begin{array}{l}\text { Iron } \\
\text { (Type II) }\end{array}$ & Civfaq, Disko & NW & 1872 & $\left.82.40^{1}\right)$ & & & & & & $\left.2.9^{2}\right)$ & 0.2 \\
\hline 228 & $\begin{array}{l}\text { Iron } \\
\text { (Type III) }\end{array}$ & Uivfaq, Disko & NW & 1872 & $\left.70.10^{1}\right)$ & & & & & & $\left.4.7^{2}\right)$ & \\
\hline
\end{tabular}


Analyses of Telluric Iron.

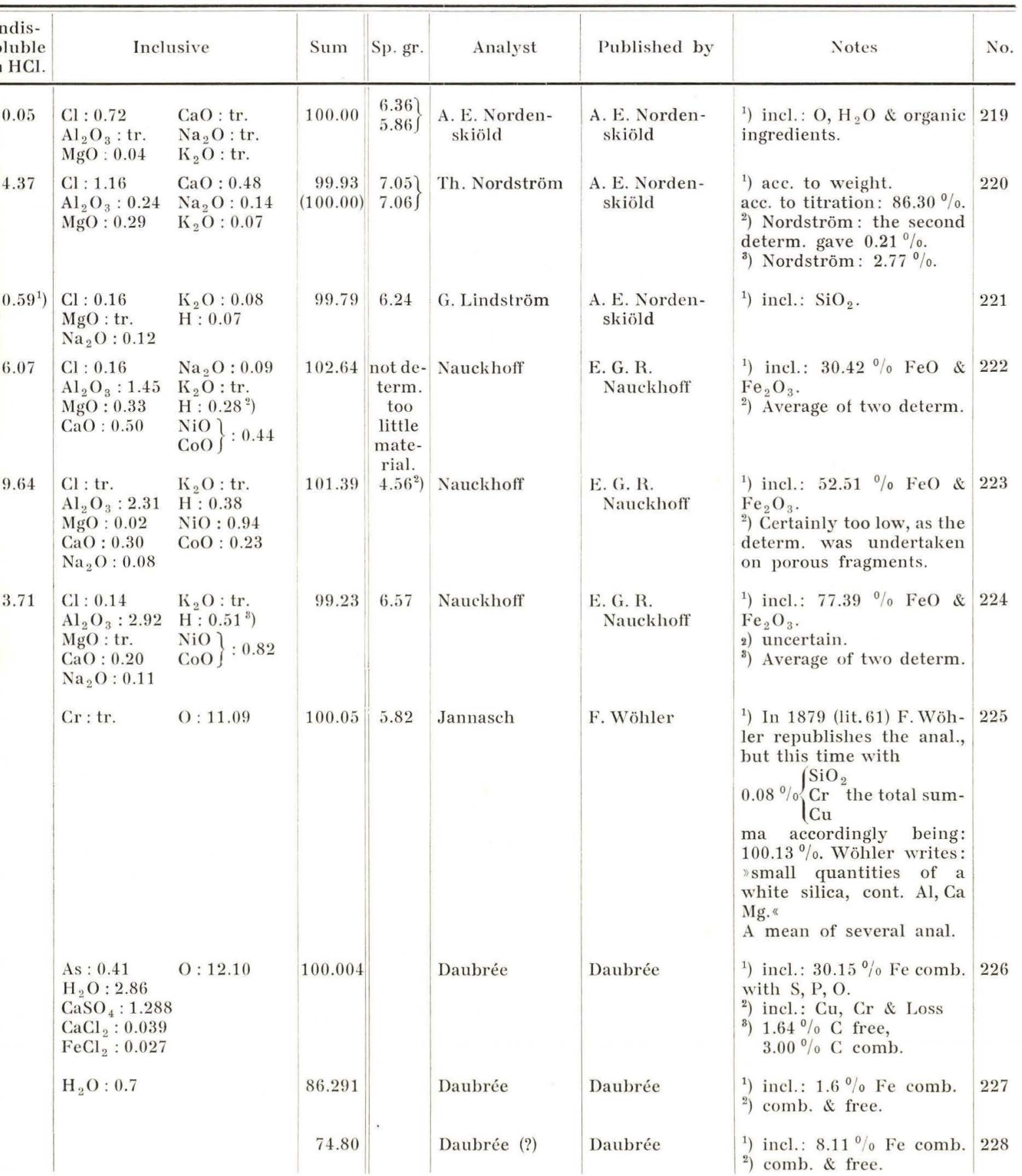




\begin{tabular}{|c|c|c|c|c|c|c|c|c|c|c|c|c|}
\hline No. & Rock Name & Locality & District & Year & $\mathrm{Fe}$ & $\mathrm{Ni}$ & Co & $\mathrm{Cu}$ & S & $\mathrm{P}$ & C & $\mathrm{SiO}$ \\
\hline 229 & $\begin{array}{l}\text { Iron } \\
\text { (Type 1) }\end{array}$ & Uivfaq, Disko & NW & 1879 & $\left.92.77^{1}\right)$ & 1.08 & 0.48 & 0.08 & 1.12 & 0.14 & 1.36 & \\
\hline 230 & $\begin{array}{l}\text { Iron } \\
\text { (Type II) }\end{array}$ & Uivfaq, Disko & NW & 1879 & 93.16 & 2.01 & 0.80 & 0.12 & 0.41 & 0.32 & $\left.2.34^{1}\right)$ & \\
\hline 231 & $\begin{array}{l}\text { Iron } \\
\text { (Type II) }\end{array}$ & Uivfaq, Disko & NW & 1879 & 90.17 & 6.50 & 0.79 & 0.13 & $\left.{ }^{1}\right)$ & 1) & 1) & 1.5 \\
\hline 232 & $\begin{array}{l}\text { Iron } \\
\text { (Type III) }\end{array}$ & Uivfaq, Disko & NW & 1879 & 88.13 & 2.13 & 1.07 & 0.48 & 0.36 & 0.25 & $\left.2.33^{1}\right)$ & 4.2 \\
\hline 233 & Iron & Uivfaq, Disko & NW & 1883 & 91.71 & 1.74 & 0.53 & 0.16 & 0.10 & nil & $\left.1.37^{1}\right)$ & 0.3 \\
\hline 234 & Iron & Uivfaq, Disko & NW & 1883 & 91.17 & 1.82 & 0.51 & $\left.0.16^{1}\right)$ & 0.78 & nil & $\left.1.70^{2}\right)$ & 0.4 \\
\hline 235 & $\begin{array}{l}\text { Iron } \\
\text { (weathered part) }\end{array}$ & Uivfaq, Disko & NW & 1883 & 82.02 & 1.39 & 0.76 & 0.19 & 0.08 & nil & $\left.1.27^{1}\right)$ & 0.5 \\
\hline 236 & $\begin{array}{l}\text { Iron } \\
\text { (the fresh, outer } \\
\text { part) }\end{array}$ & Uivfaq, Disko & NW & 1883 & 59.77 & 1.60 & 0.39 & 0.23 & & & 1.20 & 0.3 \\
\hline 237 & a. Iron & Uivfaq, Disko & NW & 1940 & 91.61 & 1.87 & & & 1.07 & & 3.48 & \\
\hline 238 & b. Iron & Uivfaq, Disko & NW & 1940 & 91.63 & 1.78 & & & 1.14 & & $\left.3.59^{1}\right)$ & \\
\hline 239 & c. Iron & Uivfaq, Disko & NW & 1940 & 91.50 & 1.76 & & & 1.04 & & $\left.3.56^{1}\right)$ & \\
\hline 240 & d. Iron & Uivfaq, Disko & NW & 1940 & 91.58 & 1.82 & & & 1.09 & & $\left.3.57^{1}\right)$ & \\
\hline 241 & e. Iron & Uivfaq, Disko & NW & 1940 & 91.72 & 1.84 & & & 1.09 & & 3.92 & \\
\hline 242 & Iron & Asuk, Disko & NW & 1883 & 95.15 & 0.34 & 0.06 & 0.14 & $\left.{ }^{1}\right)$ & & 0.96 & 0.6 \\
\hline 243 & Iron & $\begin{array}{l}\text { Jernpynten, } \\
\text { Mellemfjord, Disko }\end{array}$ & NW & 1883 & 92.41 & 0.45 & 0.18 & 0.48 & tr. & & $\left.0.87^{1}\right)$ & 0.9 \\
\hline 244 & Iron & $\begin{array}{l}\text { The head of Mellem- } \\
\text { fjord, Disko }\end{array}$ & NW & 1883 & 93.89 & 2.55 & 0.54 & 0.33 & 0.20 & nil & 0.28 & 0.4 \\
\hline 245 & $\begin{array}{l}\text { Iron } \\
\text { (Pebble) }\end{array}$ & Fortunebay, Disko & NW & 1883 & 92.68 & 2.54 & 0.58 & 0.20 & 0.01 & nil & $\left.2.40^{1}\right)$ & 0.3 \\
\hline
\end{tabular}




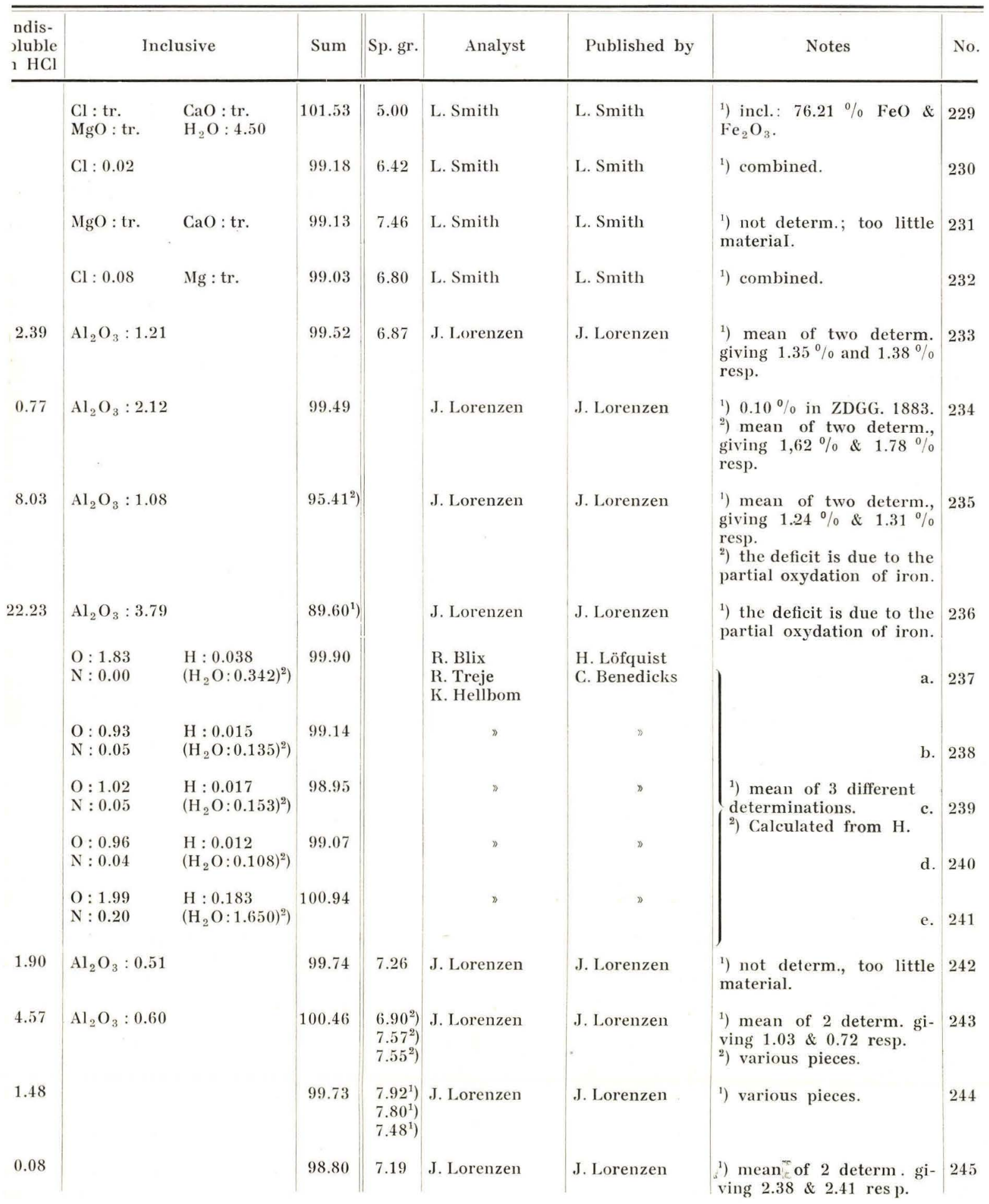




\begin{tabular}{|c|c|c|c|c|c|c|c|c|c|c|c|c|}
\hline No. & Rock Name & Locality & District & Year & $\mathrm{Fe}$ & $\mathrm{Ni}$ & Co & $\mathrm{Cu}$ & $\mathrm{S}$ & $\mathbf{P}$ & $\mathrm{C}$ & $\mathrm{SiO}_{2}$ \\
\hline 246 & $\begin{array}{l}\text { Iron } \\
\text { (Pebble) }\end{array}$ & $\begin{array}{l}\text { Niaqornaq, } \\
\text { Jakobshavn district }\end{array}$ & NW & 1854 & 93.39 & 1.56 & 0.25 & 0.45 & 0.67 & 0.18 & 1.69 & 0.38 \\
\hline 247 & $\begin{array}{l}\text { Iron } \\
\text { (Pebble) }\end{array}$ & $\begin{array}{l}\text { Jakobshavn, on a plain } \\
\text { at the river Anoretok } \\
\text { at Niaqornaq }\end{array}$ & NW & 1879 & 92.45 & 2.88 & 0.43 & 0.18 & 1.25 & 0.24 & $\left.1.74^{1}\right)$ & 1.31 \\
\hline 248 & $\begin{array}{l}\text { Iron } \\
\text { (Pebble) }\end{array}$ & $\begin{array}{l}\text { Niaqornaq } \\
\text { Anoretok river }\end{array}$ & NW & 1883 & 92.46 & $\left.1.92^{1}\right)$ & $\left.0.93^{1}\right)$ & 0.16 & 0.59 & 0.07 & $\left.3.11^{2}\right)$ & 0.24 \\
\hline 249 & $\begin{array}{l}\text { Iron } \\
\text { (Pebble) }\end{array}$ & $\begin{array}{l}\text { Niaqornaq } \\
\text { Anoretok river }\end{array}$ & NW & 1897 & 93.64 & 2.00 & 0.48 & 0.07 & 1.13 & 0.19 & 3.72 & \\
\hline 250 & $\begin{array}{l}\text { Iron } \\
\text { (Pebble) }\end{array}$ & $\begin{array}{l}\text { Niaqoruaq } \\
\text { Anoretok river }\end{array}$ & NW & 1897 & 91.60 & 1.25 & 0.37 & & & 0.07 & 6.44 & \\
\hline 251 & $\begin{array}{l}\text { Iron } \\
\text { (Pebble) }\end{array}$ & $\begin{array}{l}\text { Ekaluit, Nûgssuaq, } \\
\text { in an old greenland } \\
\text { tomb }\end{array}$ & NW & 1883 & 94.11 & 2.85 & 1.07 & 0.23 & 1) & $\left.{ }^{1}\right)$ & 1) & \\
\hline 252 & $\begin{array}{l}\text { Iron } \\
\text { (Knife) }\end{array}$ & $\begin{array}{l}\text { Sermermiut, } \\
\text { Jakobshavn }\end{array}$ & NW & 1883 & & 7.76 & 0.56 & tr. & & & & \\
\hline 253 & $\begin{array}{l}\text { Iron } \\
\text { (Knife) }\end{array}$ & Hunde Ejland & NW & 1883 & & 0.2 & & 0.18 & & & & \\
\hline 254 & $\begin{array}{l}\text { Iron } \\
\text { (Pebble) }\end{array}$ & $\begin{array}{l}\text { Arveprinsens Ejland, } \\
\text { in a moor }\end{array}$ & NW & 1883 & 95.67 & & $\operatorname{tr} . ?$ & 0.06 & 0.09 & & 1.94 & 1.40 \\
\hline 255 & $\begin{array}{l}\text { Iron } \\
\text { (Pebble) }\end{array}$ & Fiskernæs & SW & 1883 & 92.23 & 2.73 & 0.84 & 0.36 & $\left.{ }^{1}\right)$ & nil. & 0.20 & 0.64 \\
\hline 256 & $\begin{array}{l}\text { "Pyrite } \\
\text { nickelifère" }\end{array}$ & Igdlokunguaq & NW & 1879 & 53.01 & 3.11 & 0.78 & 2.43 & 36.85 & 0.42 & & \\
\hline
\end{tabular}

Analyses of Meteoric Iron.

Appendi

\begin{tabular}{|c|c|c|c|c|c|c|c|c|c|c|c|c|}
\hline No. & $\begin{array}{l}\text { Name } \\
\text { of Meteorite }\end{array}$ & Locality & District & Year & $\mathrm{Fe}$ & $\mathrm{Ni}$ & Co & $\mathrm{Cu}$ & $\mathrm{S}$ & $\mathrm{P}$ & C & $\mathrm{SiO}_{2}$ \\
\hline 257 & $\begin{array}{l}\text { "Ahnighto" } \\
\text { (Tent) }\end{array}$ & Savigsivik & NW & 1898 & 91.476 & 7.785 & 0.533 & 0.014 & nil & 0.202 & 0.014 & \\
\hline 258 & "Woman" & Ironstone Mt. & NW & 1898 & 91.468 & 7.775 & 0.533 & 0.018 & nil & 0.188 & 0.026 & \\
\hline 259 & "Dog" & Ironstone Mt. & NW & 1898 & 90.993 & 8.265 & 0.533 & 0.016 & 0.019 & 0.172 & 0.014 & \\
\hline 260 & "Savik" & Savik & NW & 1927 & n. d. & 7.25 & 0.00 & 0.00 & 0.022 & 0.166 & 0.00 & \\
\hline
\end{tabular}




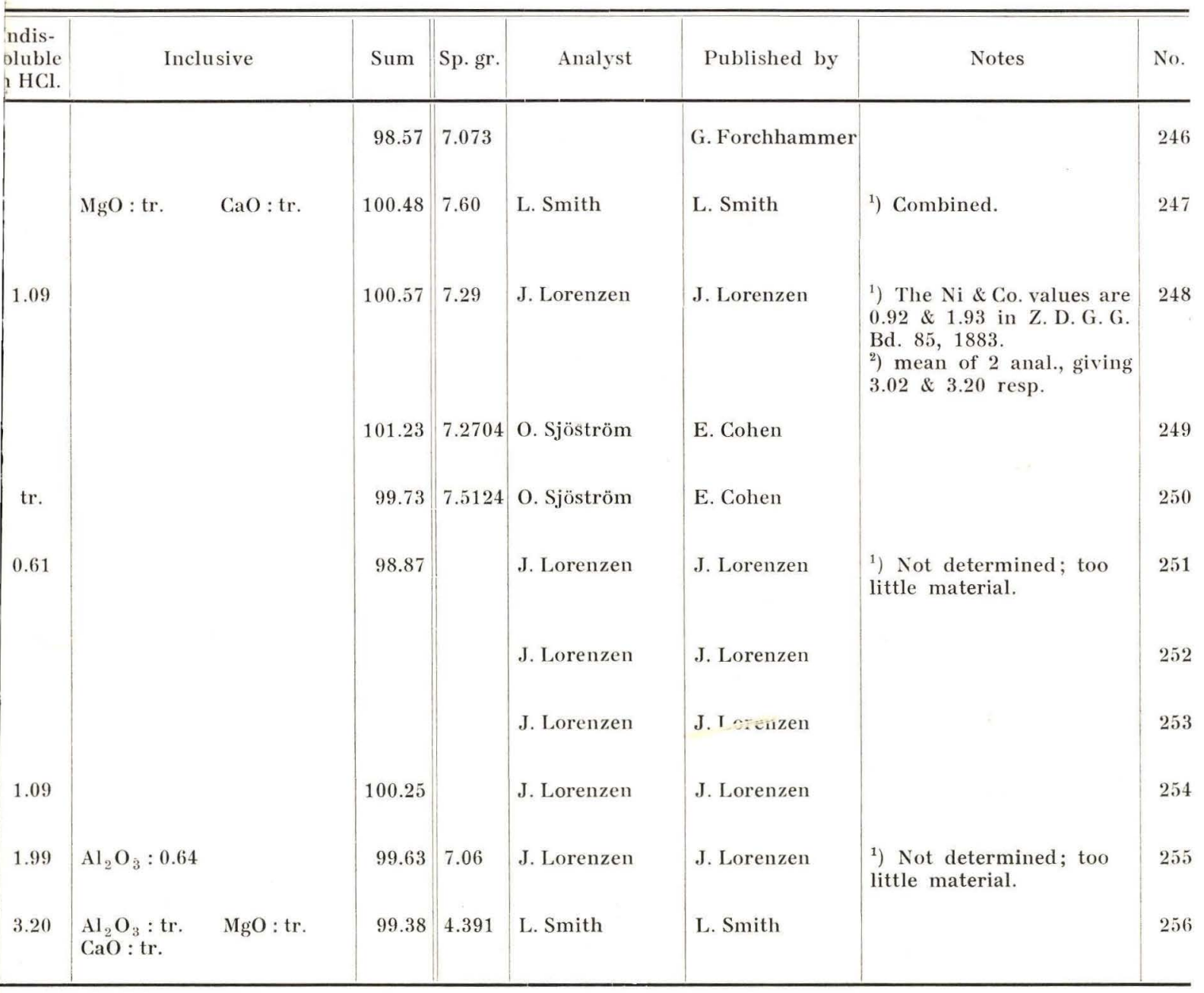

No. 2.

Analyses of Meteoric Iron.

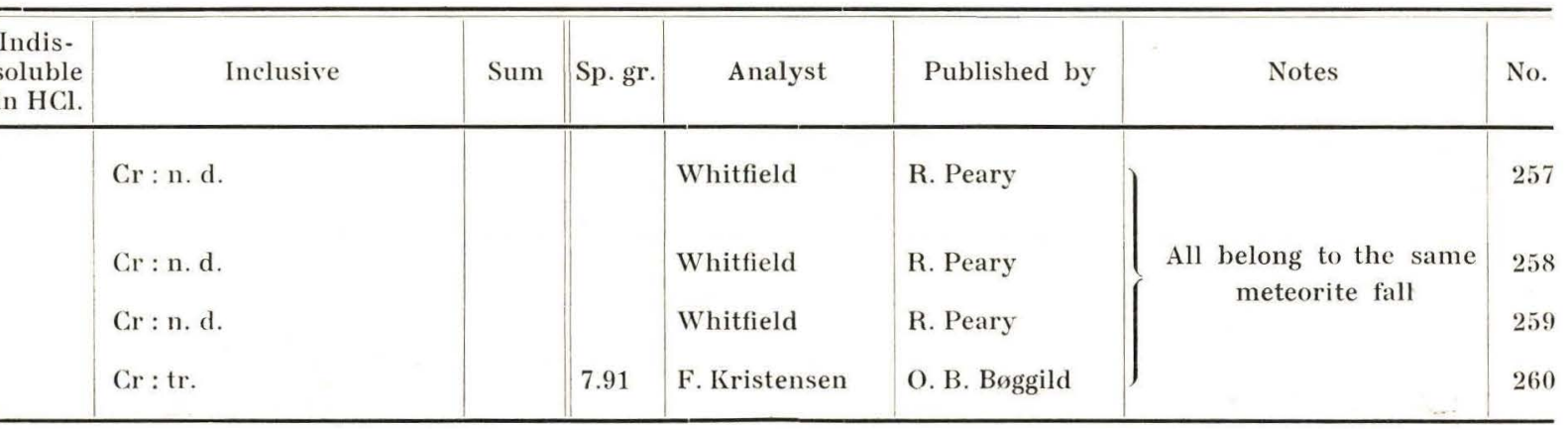




\section{List of Localities.}

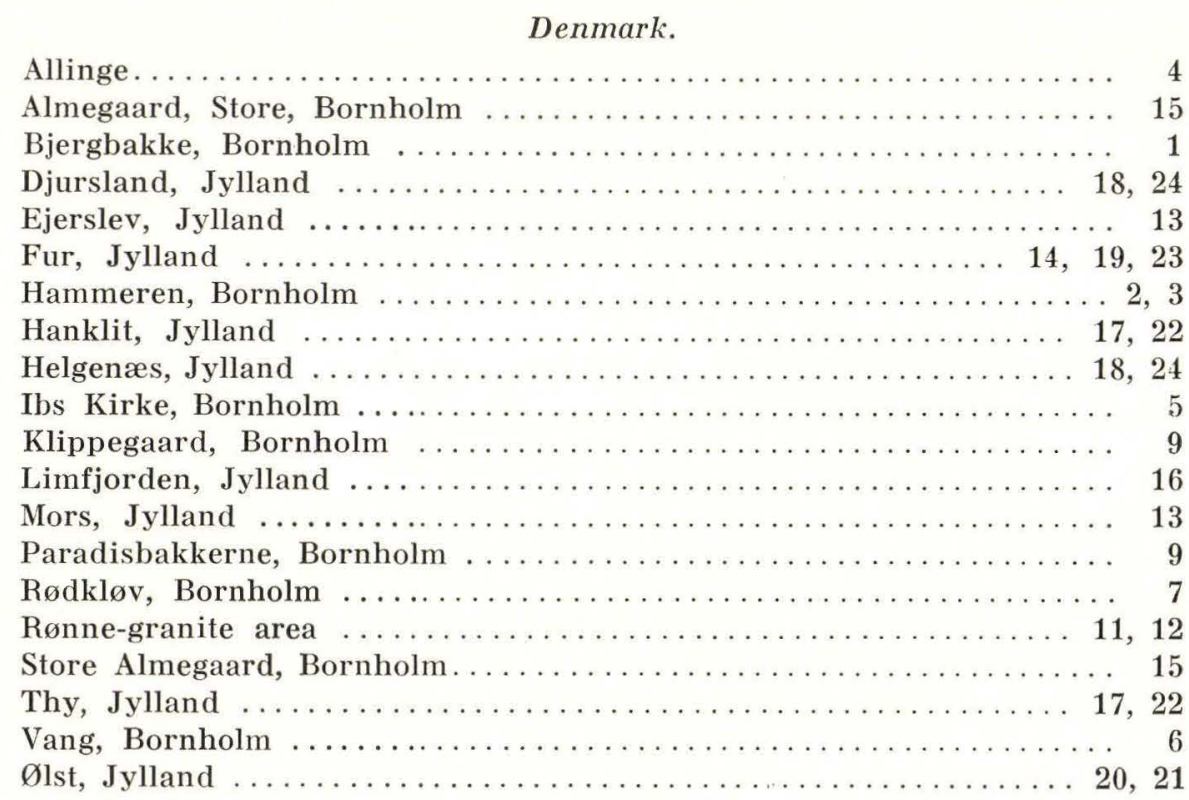

The Faeroes.

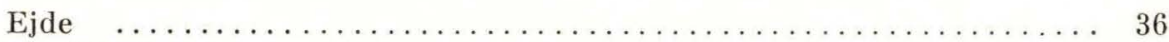

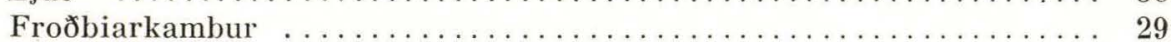

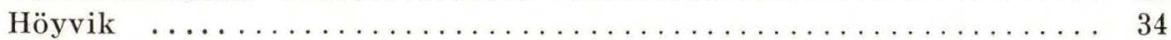

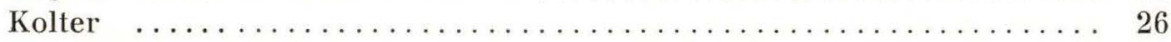

Strömø $\ldots \ldots \ldots \ldots \ldots \ldots \ldots \ldots \ldots 25,28,28$ a, 30, 31, 32, 33, 34, 37

Suðеrø $\ldots \ldots \ldots \ldots \ldots \ldots \ldots \ldots \ldots \ldots \ldots \ldots \ldots \ldots \ldots \ldots \ldots \ldots \ldots \ldots \ldots \ldots \ldots \ldots \ldots \ldots, 29,35$

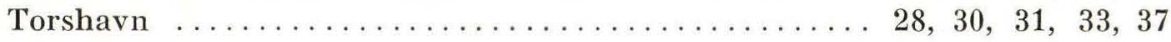

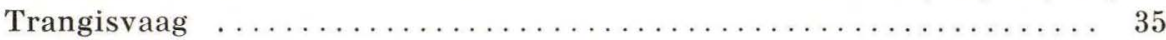

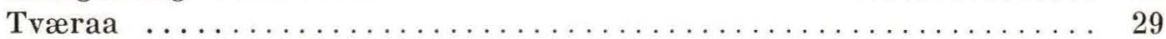

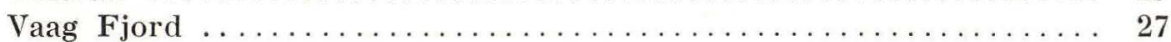

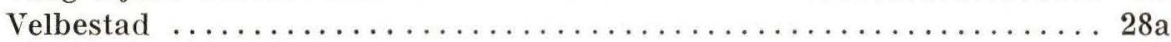

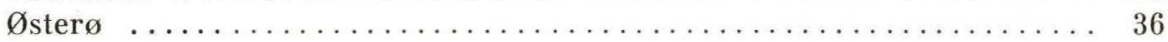


NW. Greenland.

Arfertuarssuk Fjord (Svartenhuk Peninsula) ............... 85

Arveprinsens Ejland .............................. 254

Asuk (Disko) ................................. 108, 242

Brededal (Disko) ................................... 133

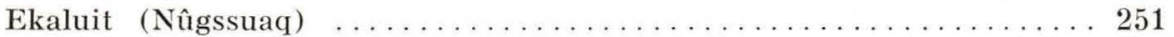

Fortunebay (west of Godhavn) . . . . . . . . . . . . . . . . 245

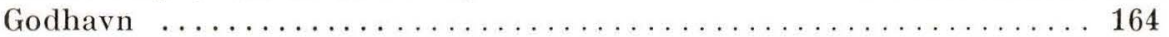

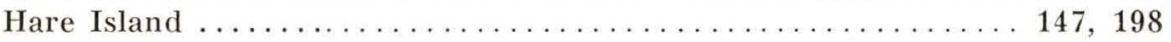

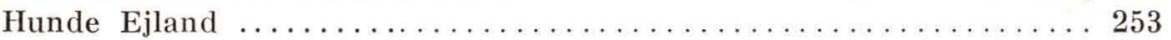

Igdlokunguaq (Disko) . . . . . . . . . . . . . . . . . . . . . . 256

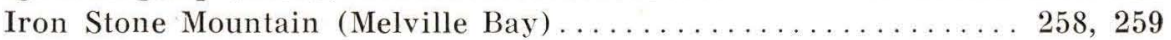

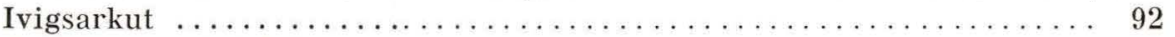

Jernpynten (Disko) ........................ 94, 243

Lyngmarksfjeld (at Godhavn) .... 166, 175, 176, 199, 202, 205, 211, 218

Mellemfjord, head of (Disko) .................... 117, 244

Niaqornaq (Jakobshavn district) ........... 246, 247, 248, 249, 250

Nûgssuaq Peninsula ........................... 76

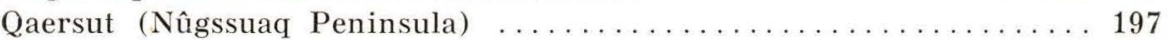

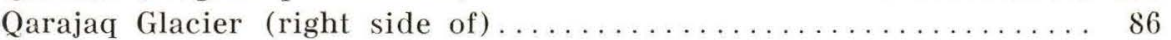

Savigsivik (Melville Bay) ........................... 257

Savik (Melville Bay) ............................. 260

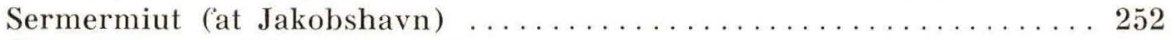

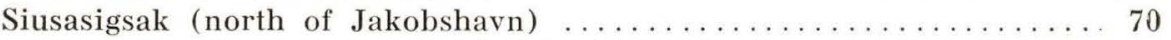

Uivfaq (Disko) ..... 135, 144, 148, 150, 152, 160, 169, 183, 191, 196, $201,204,207,210,212,214,219,220,221,223,224,225,226$, $227,228,229,230,232,233,234,235,236,237,238,239,240,241$

Umanak Island ........................ 45, 69, 156

Umîvik Fjord (Svartenhuk Peninsula) . . . . . . . . . . . . . 187

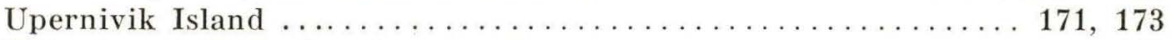

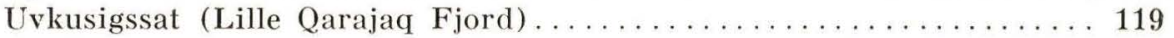

Østerfjeld (Qaersut) $\ldots \ldots \ldots \ldots \ldots \ldots \ldots \ldots \ldots \ldots . \ldots 1,141,190,203,209$

\section{SW.-Greenland.}

Akuliarusek (Igaliko Fjord) . . . . . . . . . . . . 101, 109, 123

Fiskernæs (south of Godthaab) .................. 127, 225

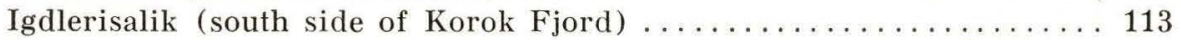

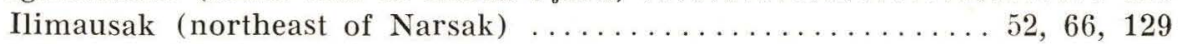

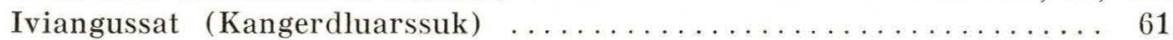

Ivigtut Peninsula .........................44, 105, 112

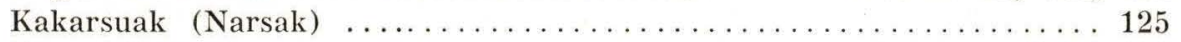

Kangerdluarsuk .......................... 126 a, 130

Kringlerne (south side of Kangerdluarsuk Fjord) ........ 122, 131, 136

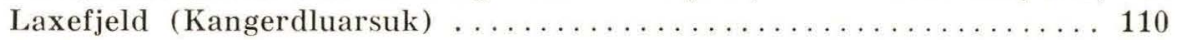

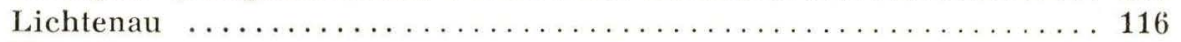

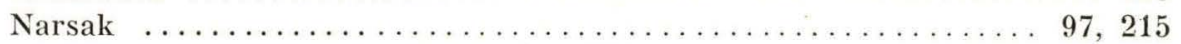

Naujakasik (south side of Tunugdliarfik) .................. 103

Niakornarsuk (northwest side of Korok Fjord) . . . . . . . . . . . . 111

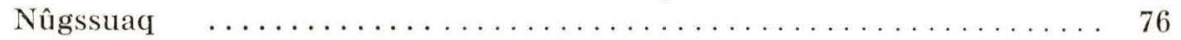

Nunasarnak (north side of Tunugdliarfik) ............. 102, 194 
Nunasarnausak (north side of Kangerdluarsuk) ........... 104, 181

Panernak Bay (northwest of Narsak) . . . . . . . . . . . . . . . . . . . 174

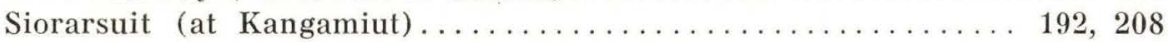

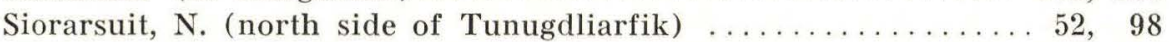

Siorarsuit, S. (south side of Tunugdliarfik) $\ldots \ldots \ldots \ldots \ldots \ldots \ldots \ldots \ldots$

Tasek (northeast of Narsak) .......................... 157

Tupersuatsiak (south side of Tunugdliarfik Fjord) ..........144, 132

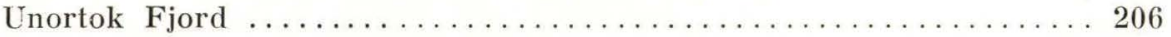

NE. Greenland.

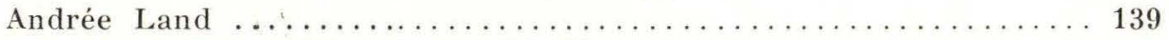

Antartic Harbour (King Oscar Fjord) . . . . . . . . . . . . 87

Bastionen (Ella Island) .............................. 124

Cape Fletcher (Canning Land) $\ldots \ldots \ldots \ldots \ldots \ldots \ldots 46,90,93,186$

Cape Franklin (Gauss Peninsula)...... 38, 39, 48, 51, 53, 56, 63, 72, 167

Cape Graah (Ymer Island) ........................ 41

Cape Hope (Liverpool Land) . . . . . . . . . . . . . . . . . . . . . . 84

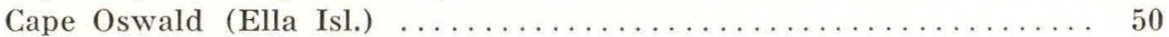

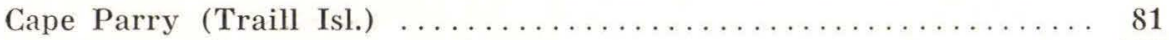

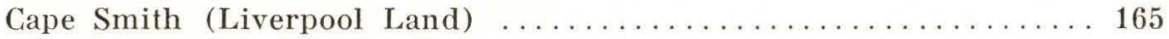

Cape Weber (Andrée Land) ......................... 73

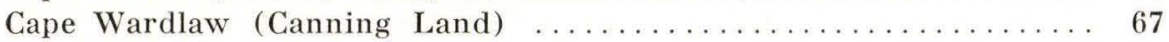

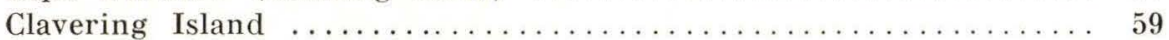

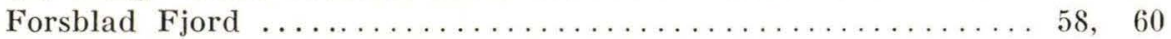

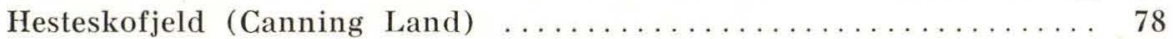

Hodal (Liverpool Land) .......................... 106

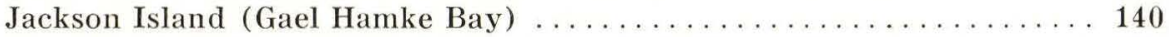

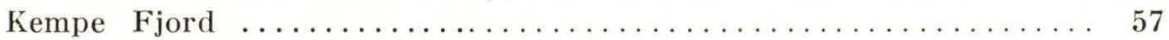

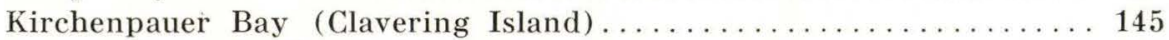

Ladder Mountain (head of Musk Ox Fjord) . . . . . . . . . . . . . . 195

Liverpool Land ................. 40, 62, 65, 115, 138, 178, 213

Mt. Högbom (Gauss Peninsula) . . . . . . . . . . . . . . . 44

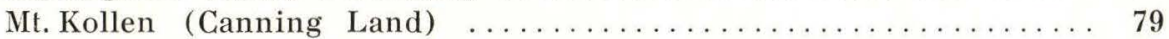

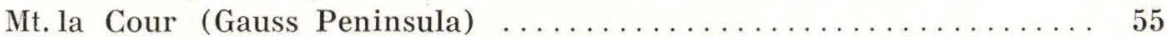

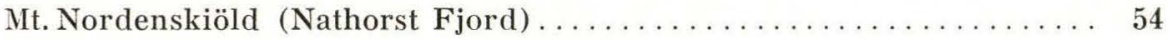

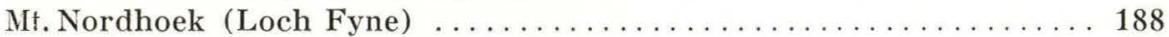

Mt. Ramsay (Gauss Peninsula) . . . . . . . . . . . . . . 43, 193

Musk Ox Fjord ............................... 42, 64

Neills Cliff (Jameson Land) . . . . . . . . . . . . . . . . . 168

Porfyrfjeld (Canning Land) $\ldots \ldots \ldots \ldots \ldots \ldots \ldots \ldots$ 80, 83, 89, 120

Raffles Island (off the Liverpool coast) ................. 99

Riddarborgen (Franz Joseph Fjord) . . . . . . . . . . . . . . 142

Segelsällskapets Fjord $\ldots \ldots \ldots \ldots \ldots \ldots \ldots \ldots \ldots \ldots \ldots \ldots \ldots$ 82, 161

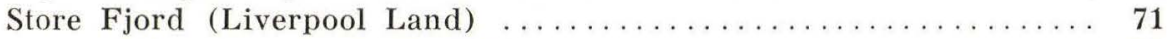

Tvekegledal (Wegener Peninsula) ....................... 75

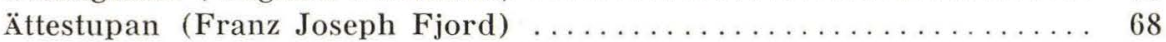

Øvre Rypegletcher (Franz Joseph Fjord) ................... 77

SE. Greenland.

Barclay Bay, north side of .......................... 159

Basishusene (Kangerdlugssuaq) . . . . . . . . . . . . . . 184 
Basistoppen (Kangerdlugssuaq) .......... 107, 121, 182, 185, 189

Brødretoppen (Kangerdlugssuaq) ................ 95, 134, 149

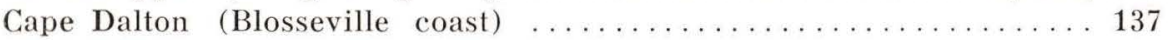

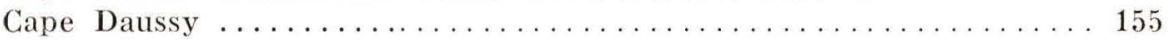

Forbindelsesgletcher (Kangerdlugssuaq) . . . . . . . . . . . . . 143

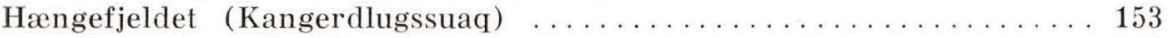

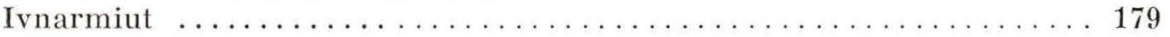

Kangerdlugssuaq, west side of $\ldots \ldots \ldots \ldots \ldots \ldots \ldots \ldots \ldots \ldots \ldots$ 88, 118

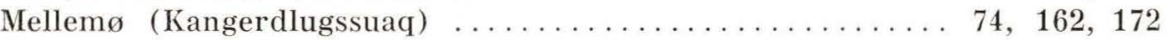

Mikis Fjord, south side of (near Kangerdlugssuaq) . . . . . . . . . 158

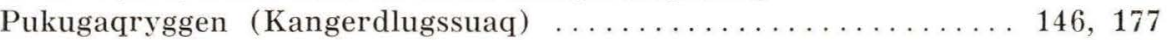

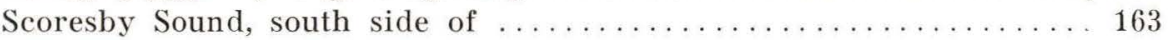

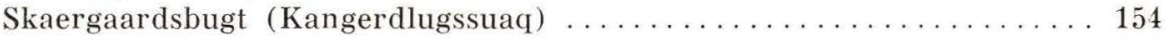

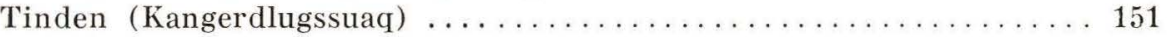

Uttentals Plateau (Kangerdlugssuaq) ................ 170, 180, 200

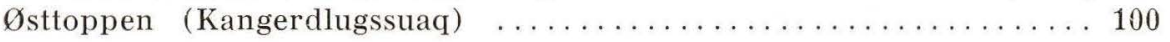




\section{Index of Rock Names.}

(Names used by the authors.)

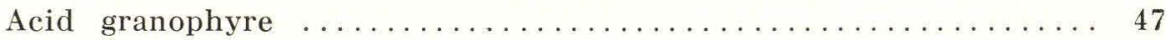

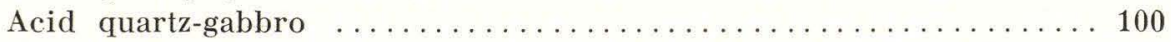

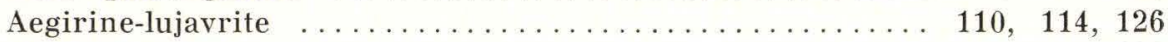

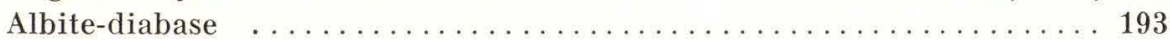

Alkali-granite ............................... 49

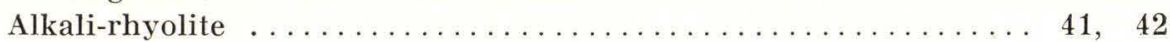

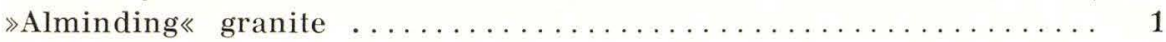

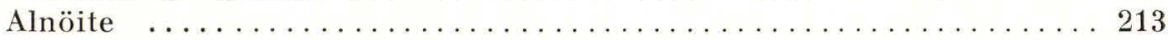

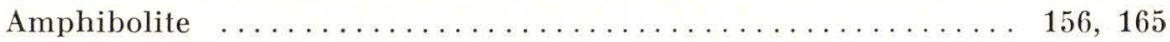

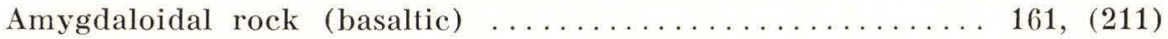

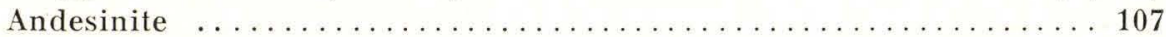

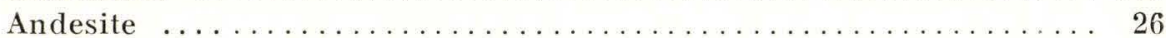

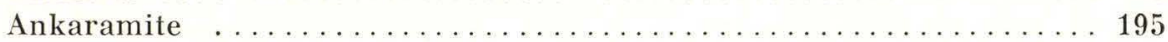

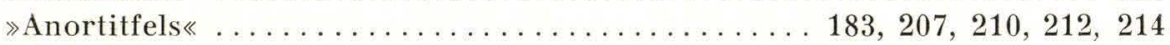

Anorthoclase-trachyte ......................... 85

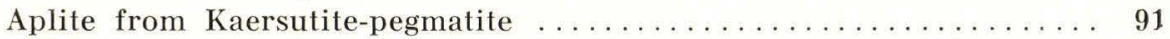

Aplite-granite .............................. 44

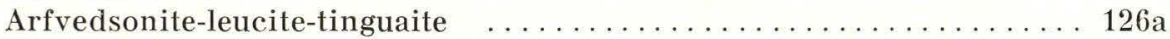

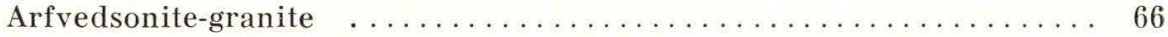

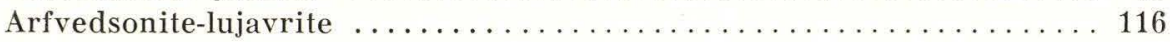

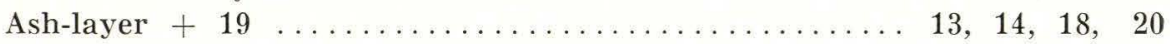

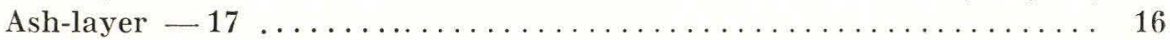

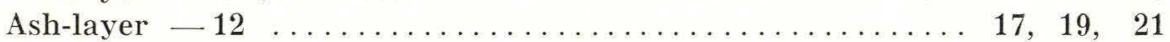

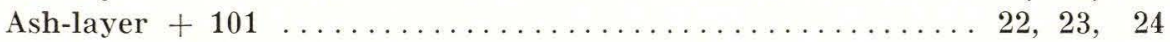

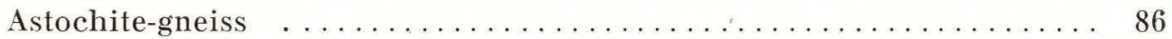

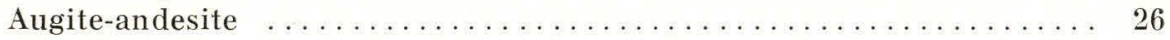

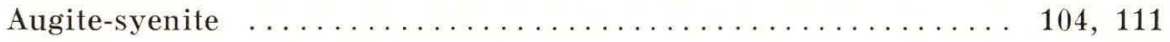

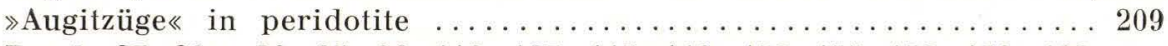

Basalt 27, 28 a, 30, 35, 36, 118, 137, 147, 148, 153, 155, 158, 159, 163,

$164,166,167,168,175,176,187,191,196,198,199,201,202,205$

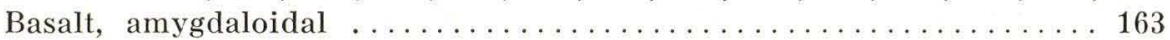

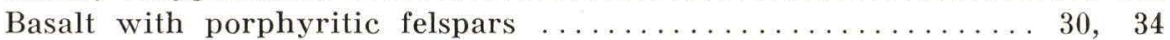

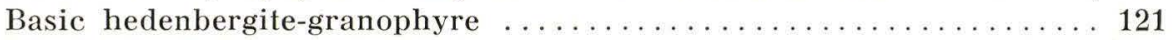

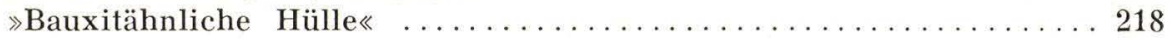

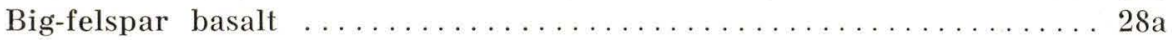

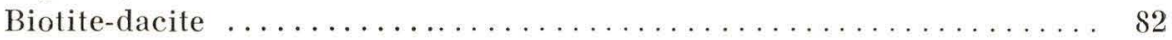




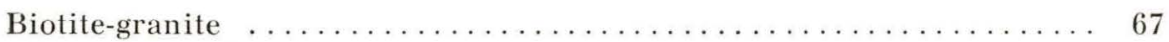

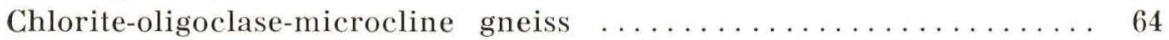

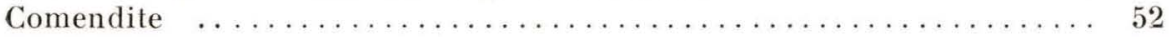

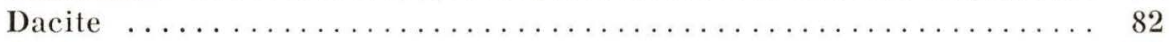

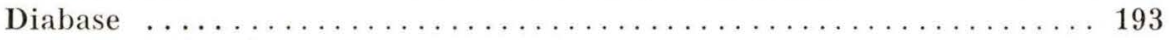

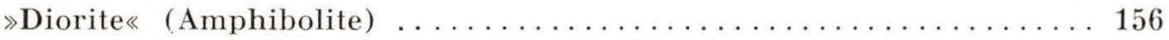

Dolerite 29, 108, 117, 127, 133, 135, 140, 144, 145, 150, 152, 160, 169, 190, 204

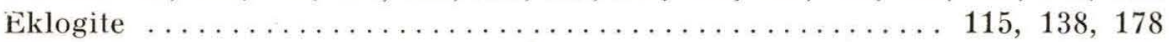

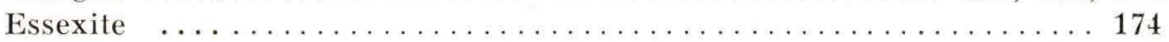

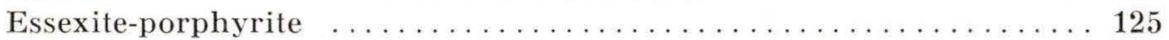

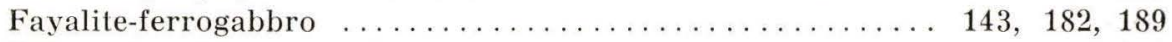

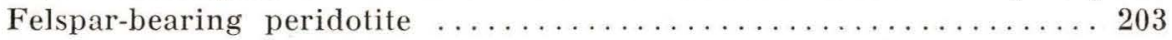

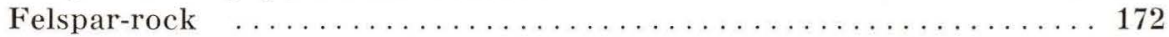

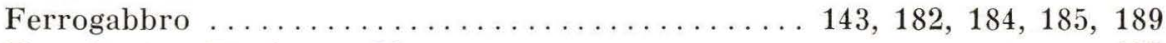

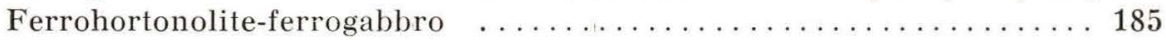

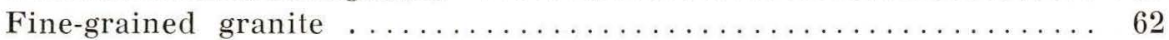

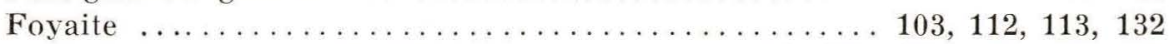

Gabbro ....... 100, 128, 134, 146, 149, 151, 154, 162, 170, 177, 179, 180

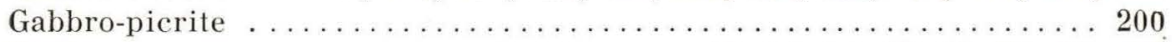

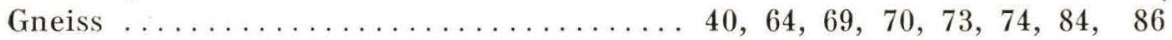

Granite... 1, 2, 3, 4, 5, 6, 7, 8, 9, 10, 11, 12, 15, 44, 45, 49, 50, 55, 57, $58,59,60,61,62,65,66,67,68,71,77,80$

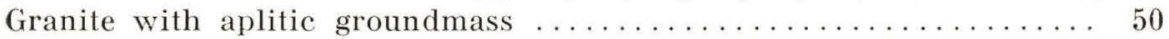

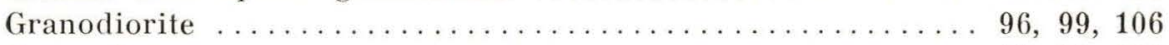

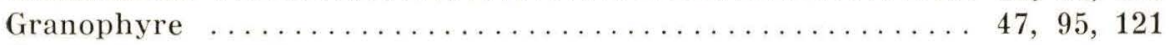

Granophyre-granite $\ldots \ldots \ldots \ldots \ldots \ldots \ldots \ldots \ldots \ldots \ldots \ldots \ldots \ldots$

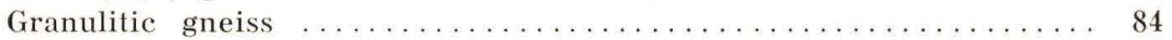

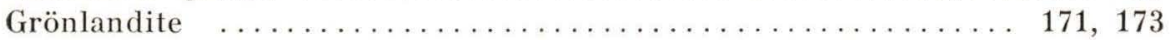

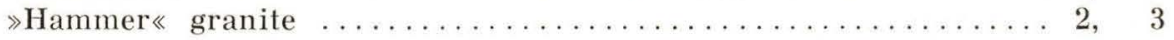

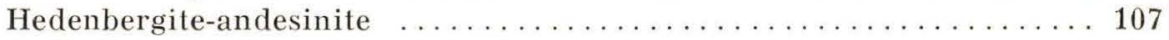

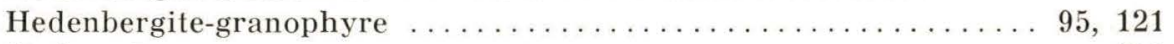

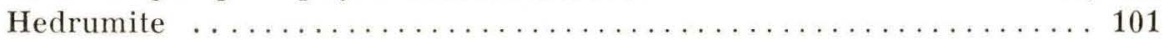

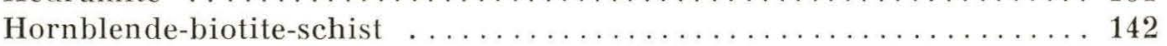

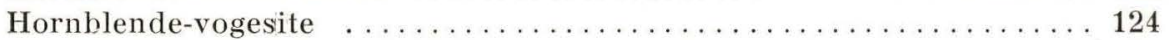

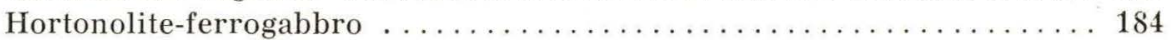

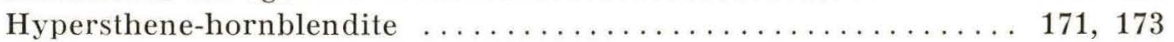

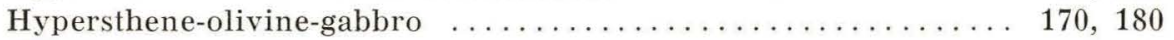

$\gg$ Ilimausak

Iron 219, 220, 221, 222, 223, 224, 225, 226, 227, 228, 229, 230, 231, 232, $233,234,235,236,237,238,239,240,241,242,243,244,245,246,247$, $248,249,250,251,252,253,254,255$, and $257,258,259,250$

Iron-bearing basalt $\ldots \ldots \ldots \ldots \ldots \ldots \ldots \ldots \ldots \ldots \ldots$ 148, 191, $196 \ldots \ldots \ldots$ Iron-bearing dolerite $\ldots \ldots \ldots \ldots$ 108, 117, 127, 144, 152, 160, 169, 204 Iron-bearing rock (basaltic) $\ldots \ldots \ldots \ldots \ldots \ldots \ldots \ldots \ldots \ldots \ldots, 94$

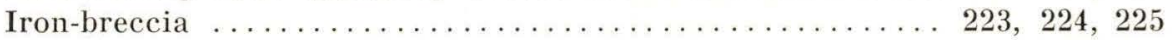

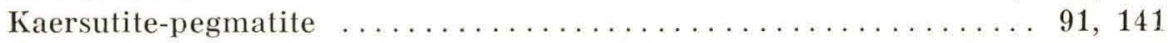

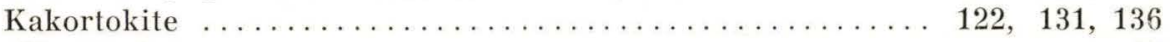

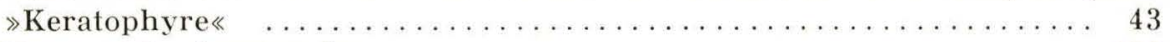

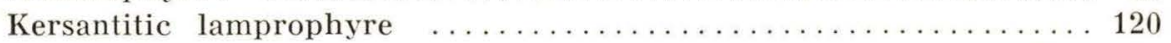

Lamprophyre .............................. 120

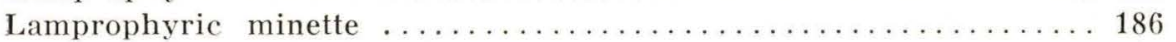


Leuko-syenite . . . . . . . . . . . . . . . . . . . . . . . . . . . . 105

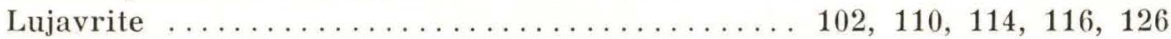

Magnesite-pyroxenite . . . . . . . . . . . . . . . . . . . . 215

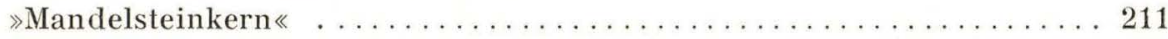

Meteoric iron ...................... 257, 258, 259, 260

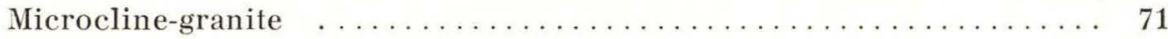

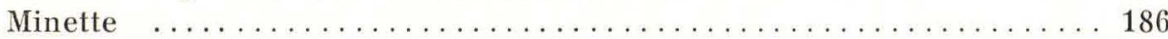

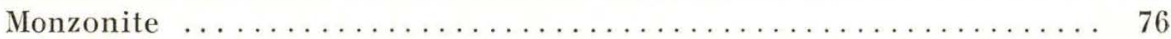

Naujaite .............................. 130, 194

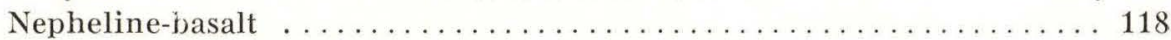

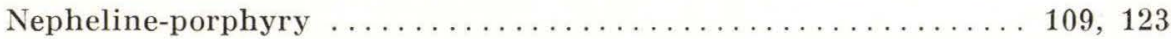

Nepheline-sodalite-syenite $\ldots \ldots \ldots \ldots \ldots \ldots \ldots \ldots \ldots \ldots \ldots \ldots . \ldots \ldots$

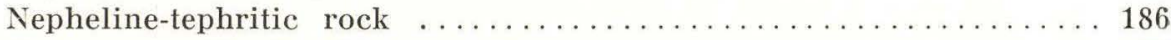

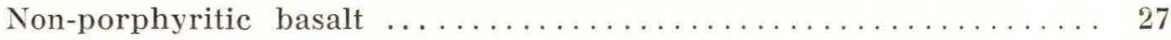

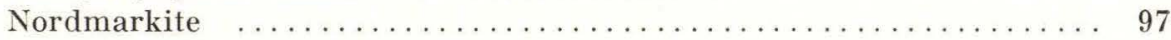

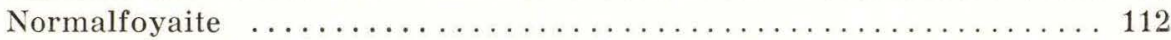

Olivine-basalt $\ldots \ldots \ldots \ldots \ldots \ldots \ldots \ldots \ldots .35,36,155,158,159,187,198$

Olivine-dolerite . . . . . . . . . . . . . . . . . . . . . . . 29

Olivine-gabbro $\ldots \ldots \ldots \ldots \ldots \ldots \ldots \ldots \ldots$ 128, 151, 154, 162, 170, 180

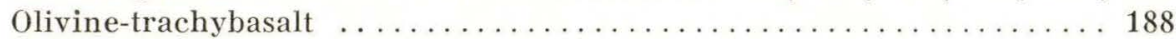

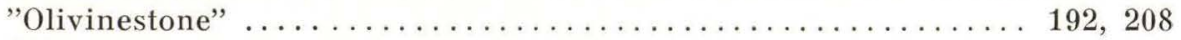

"Olivinestone", weathered ............................ 192

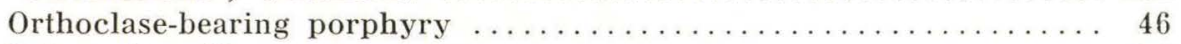

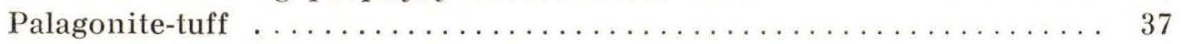

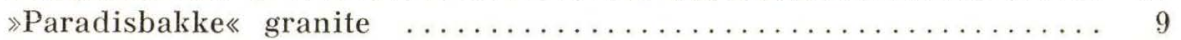

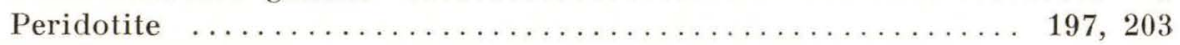

Peridotite var. picrite ............................ 197

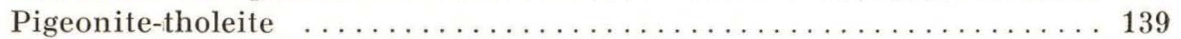

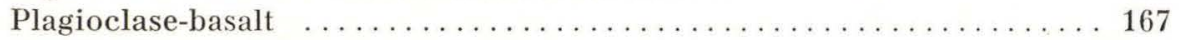

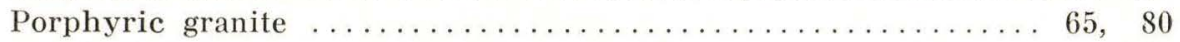

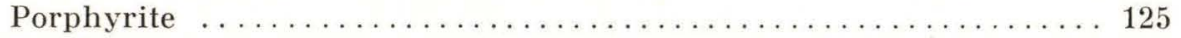

Porphyry $\ldots \ldots \ldots \ldots 46,52,54,75,78,79,83,89,90,93,109,123,129$

Porphyry granite ................................. 50

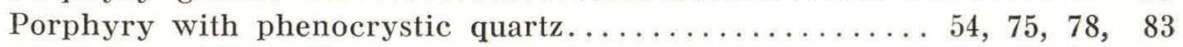

Porphyry without phenocrystic quartz.......... 79, 89, 90, 93

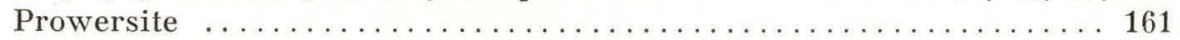

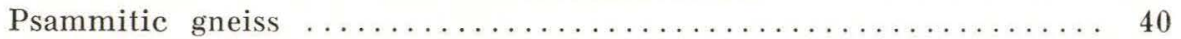

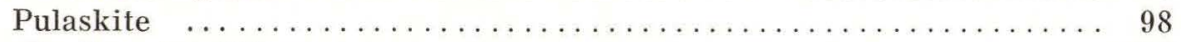

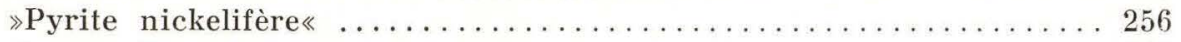

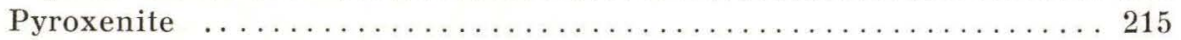

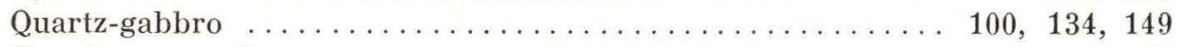

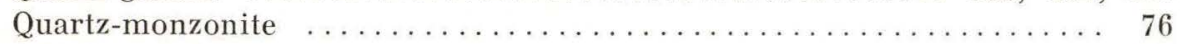

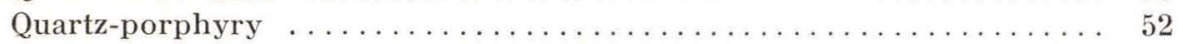

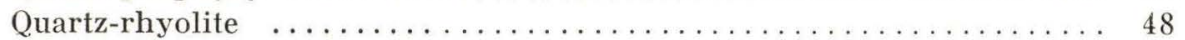

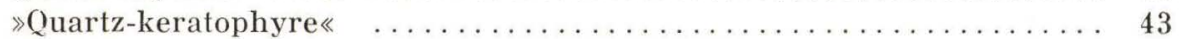

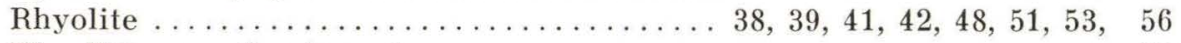

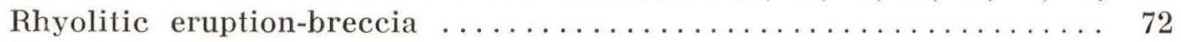

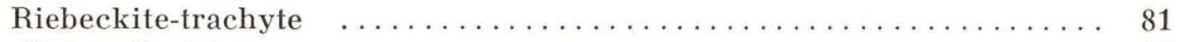

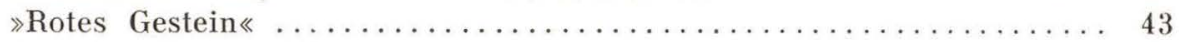

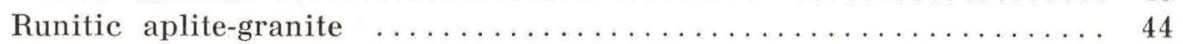

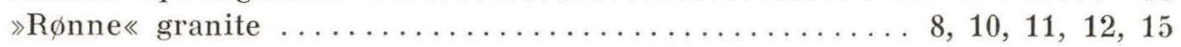


Sanidine-rhyolite

$38,39,51$

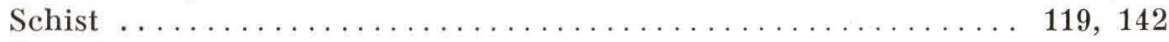

Soapstone ............................ 206, 216, 217

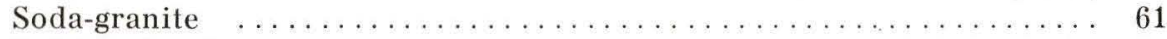

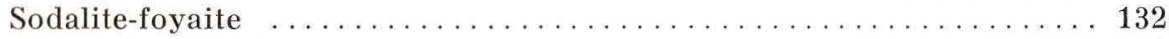

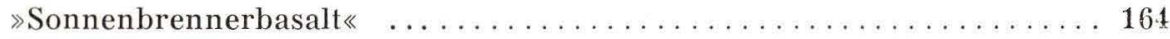

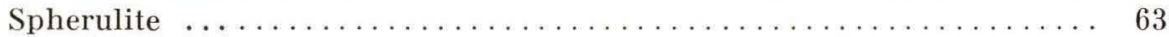

Spherulitic rhyolite $\ldots \ldots \ldots \ldots \ldots \ldots \ldots \ldots \ldots \ldots \ldots \ldots \ldots \ldots \ldots \ldots$

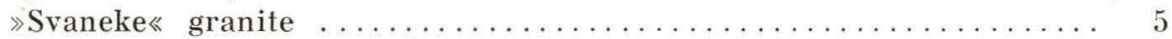

Syenite . . . . . . . . . . $\ldots \ldots \ldots \ldots \ldots \ldots \ldots \ldots$ 87, 104, 111

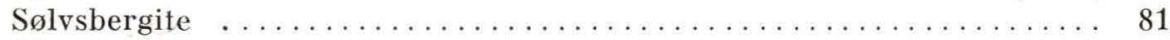

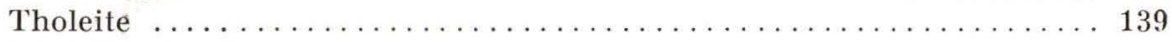

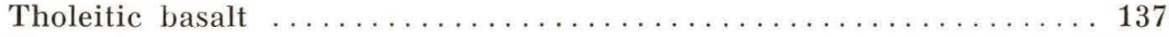

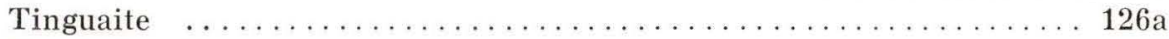

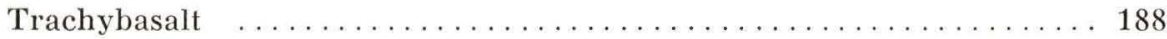

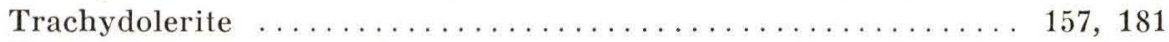

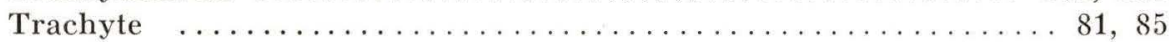

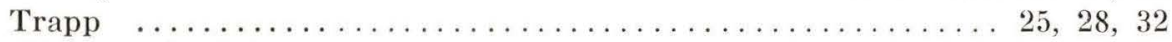

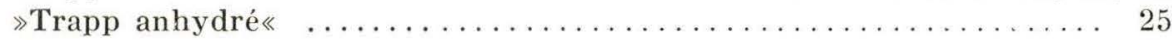

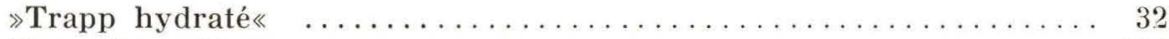

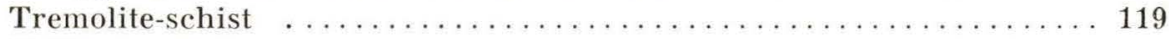

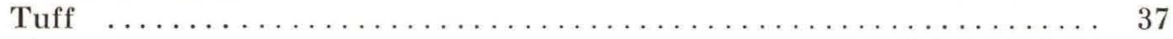

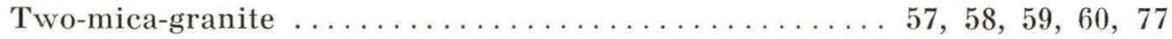

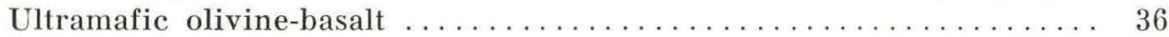

$»$ Vang granite $\ldots \ldots \ldots \ldots \ldots \ldots \ldots \ldots \ldots \ldots \ldots \ldots \ldots \ldots \ldots$

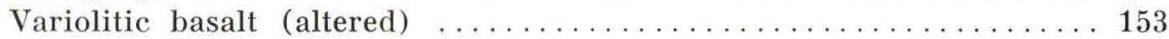

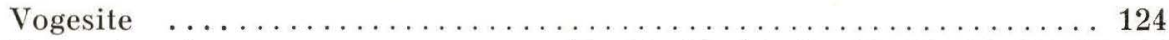

Volcanic ash .............13, 14, 16, 17, 18, 19, 20, 21, 22, 23, 24

Zeolitic amygdaloidal rock $\ldots \ldots \ldots \ldots \ldots \ldots \ldots \ldots \ldots \ldots \ldots \ldots \ldots$ 


\section{Literature.}

1. Backlund, H. G. Das Alter des »Metamorphen Komplex« von Franz Joseph Fjord in Ost-Grönland. Medd. om Grønl. Bd. 87, no. 4, Købh. 1932.

2. — \& Malmquist, D. Zur Geologie und Petrographie der nordostgrönländischen Basaltformation. Teil 1. Basische Reihe. Medd. om Grønl. Bd. 87, no. 5. Købh. 1932.

3. - - Zur Geologie und Petrographie der nordostgrönländischen Basaltformation. Teil 2. Die sauren Ergussgesteine von Kap Franklin. Medd. om Grønl. Bd. 95, no. 3 .Købh. 1935.

4. Belowsky, M. Beiträge zur Petrographie des westlichen Nord-Grönlands. Zeitschr. d. Deut. Geol. Gesellsch. Bd. 58. Berlin 1905.

Benedicks, C. (1940). Vide Löfquist \& Benedicks.

5. Bütler, H. Some new Investigations of the Devonian Stratigraphy and Tectonics of East Greenland. Medd. om Grønl. Bd. 103, no. 2. Købh. 1935.

6. Bøggild, O. B. Den vulkanske Aske i Moleret samt en Oversigt over Danmarks ældre Tertiærbjergarter. D. G. U. 2. Række, Nr. 33. Købh. 1918.

Callisen, K. (1928). Vide Madsen, Nordmann and others.

7. Callisen, K. Beiträge zur Kenntnis des Granitgrundgebirges von Bornholm. Købh. 1932.

8. - Das Grundgebirge von Bornholm. D. G. U. 2. Række, Nr. 50. Købh. 1934. (Also embracing the preceeding paper).

9. Cohen, E. Ưber das Vorkommen von Eisencarbid (Cohenit) im terrestrischen Nickeleisen von Niakornak bei Jakobshavn in NordGrönland. 1897. Medd. om Grønl. Bd. 15. Købh. 1898.

10. Daubrée. Examen des roches avec fer natif, découvertes en 1870 par M. Nordenskiöld au Groënland. Compt. Rend. des Séances de l'Acad. des sciences. Tom. 74. Paris 1872.

11. - Examen des météorites d'Ovifak (Groënland) au point de vue du carbone et des sels solubles qu'ils renferment. Compt. rend. d. Séances de l'Acad. des Sciences. Tom. 75, Paris 1872.

Davidson, Ch. (1936). Vide Walker \& Davidson.

Deer, W. A. (1939). Vide Wager \& Deer.

12. Drescher, F. K. \& Krueger, H. K. E.: Der Peridotit von Kaersut (Grönland) und sein Ganggefolge als Beispiel einer Sekretionsdifferentiation. Neues Jahrb. f. Min., Geol. u. Pal. Beil.-Band 57 A. Stuttgart 1928.

13. Durocher, M. J. Sur les roches et les minéraux des iles Féroë. Annales des Mines. Tom. 19. Paris 1841. 
14. Forchhammer, G. Meteorjærn fra det nordlige Grønland. Oversigt over d. Kgl. danske Vidensk. Selsk. Forh. No. 1. Købh. 1854.

15. Hintze, C. Handbuch der Mineralogie, 1 Band, 1. Abt. Leipzig 1904.

16. Holler, K. Rote Zersetzungszonen in grönländischen Basalten. Fortschr. d. Min., Krist. u. Petr. Bd. 17, Berlin 1933.

17. Holler, K. Hydrothermale Zersetzungserscheinungen an grönländischen Basalten. Chemie der Erde, Bd. 8. Jena 1933-34.

18. Holmes, A. The Basaltic Rocks of the Arctic Region. The Min. Mag. Vol. 18. London 1919.

19. Janovskỷ, J. Analyse zweier Grönländischer Mineralien. Berichte d. Deut. Chem. Gesellschaft zu Berlin. 1873.

20. Kalb, G. Petrographische Untersuchungen am Granit von Bornholm. Mitt. aus d. Naturwissensch. Verein f. Neuvorpommern u. Rügen in Greifswald. Jahrg. 45, 1913. Berlin 1914.

21. Kranck, E. H. On the Crystalline Complex of Liverpool Land. Medd. om Grønl. Bd. 95, no. 7. Købh. 1935.

22. Krueger, H. K. E. Úber eine sogenannte Basaltbreccie in der KreideTertiär-Formation von Westgrönland. Notizbl. d. Hessischen Geol. Landesanst. zu Darmstadt. 5 Folge, 10 Heft. 1927.

- (1928). Vide Drescher \& Kreuger.

23. Lorenzen, Joh. Kemisk Undersøgelse af det metalliske Jern fra Grønland samt nogle af de dermed følgende Bjergarter. 1882. Medd. om Grønl. Bd. 4. Købh. 1883.

24. Löfquist, H. \& Benedicks, C. Det stora Nordenskiöldska jernblocket från Ovifak, dess mikrografi och metallurgi. Jernkontorets Annaler, Årg. 124, H. 12, Stockholm 1940.

25. Machatschki, F. Enstatit-Hornblendit von Grönland. Centralblatt f. Min., Geol. u. Pal. Jahrg. 1927. Abt.-A. Stuttgart 1927.

26. Madsen, V., Nordmann, V. and others: Oversigt over Danmarks Geologi. D. G. U. 5. Række Nr. 4. Købh. 1928.

Malmquist, D. (1932, 1935). Vide Backlund \& Malmquist.

27. Nauckoff, E. G. R. Om förekomsten af gediget jern i en basaltgång vid Ovifak i Grönland. Bih. t. K. Vet.-Akad. Handlingar. Bd. 1, no. 6. Stockholm 1872.

28. Nicolau, Th. Untersuchungen an den eisenführenden Gesteinen der Insel Disko. Medd. om Grønl. Bd, 24. Købh. 1901.

29. Nieland, H. Beitrag zur Kenntnis der Deckenbasalte von Westgrönland. Chemie der Erde, Bd. 6. Jena 1931.

30. Noe-Nygaard, A. A Preliminary Report on the Palaeozoic Igneous Rocks of Canning Land. Medd. Dansk Geol. Foren. Bd. 9, H. 1. Købh. 1936.

31. - Die Palaeozoischen Eruptivgesteine von Canning-Land. Medd. om Grønl. Bd. 118, no. 6. Købh. 1937.

32. - - Syenitforekomsten ved Antarctic Havn. Østgrønland. Medd. Dansk Geol. Foren. Bd. 9, H. 5. Købh. 1940.

33. Nordenskiöld, A. E. Redogörelse för en expedition till Grönland år 1870. öfv. af K. Vet.-Akad. Förh. no. 10. Stockholm 1870.

34. Nordenskiöld, O. On the Geology and the Physical Geography of East Greenland. Medd. om Grønl. Bd. 28. Købh. 1908.

Nordmann, V. (1928). Vide Madsen, Nordmann \& others.

35. Nordström, Th. Kemisk undersökning af meteorjern från Ovifak på Grönland. öfv. af K. Vet.-Akad. Förh. no. 4, Stockholm 1871. 
36. Norin, R. Problems Concerning the Volcanic Ash Layers of the Lower Tertiary of Denmark. Geol. Fören. Förh. Bd. 62, Stockholm 1940.

37. Orvin, A. K. Beiträge zur Kenntnis des Oberdevons Ost-Grönlands. Norges Svalbards- og Ishavs-Undersøkelser. Nr. 30. Oslo 1930.

38. Osann, A. Ueber einige basaltische Gesteine der Färöer. Neues Jahrb. f. Min., Geol. u. Pal. Bd. 1. Stuttgart 1884.

38 a. Peary, R. Northward over the "Great Ice". II. 1898.

39. Phalen, W. C. Notes on the Rocks of Nugsuaks Peninsula and its Environs, Greenland. Smith. Inst. Misc. Coll. Vol. 45, (no. 1436). Washington, Philadelphia.

40. Rittmann, A. Studien an Eruptivgesteinen aus Ost-Grönland. Medd. om Grønl. Bd. 115, no. 1. Købh. 1940.

41. Rosenbusch, H. Elemente der Gesteinslehre. 1898.

42. Rørdam, K. Undersøgelse af Olivinsten fra Siorarsuit ved Kangamiut i Grønland. Medd. om Grønl. Bd. 8. Købh. 1889.

43. - Undersøgelse af mesozoiske Lerarter og Kaolin paa Bornholm i geologisk og teknisk Henseende. D. G. U. 2 Række Nr. 1. Købh. 1890.

44. - Studier over udvalgte Emner af den kemiske Geologi og den kemiske Agrogeologi. Det kgl. danske Vidensk. Selsk. Skr. 7. Række Afd. XI. 5. København 1914.

45. Sahama (Sahlstein), Th. G. Petrographie der Eklogiteinschlüsse in den Gneisen des südwestlichen Liverpool-Landes in Ostgrönland. Medd. om Grønl. Bd. 95 no. 5. Købh. 1935.

46. Simpson, J. B. Notes on the Geology of the Faeröe Islands. Geol. Magazine. Vol. 65. London 1928.

47. Smith, L. Memoire sur le fer natif du Groenland et sur la dolérite qui le renferme. Annales de chimie et de physique. 5. serie, Tom. 16. Paris 1879.

48. Streng, Aug. Beitrag zur Theorie der vulkanischen und plutonischen Gesteinsbildung. Ann. der Physik u. Chemie. Bd. 90. Leipzig 1853.

49. Tyrrell, G. W. The Petrography of some Kainozoic Igneous Rocks, and of the Cape Parry Alkaline Complex, East Greenland. Geol. Magazine. Vol. 69. London 1932.

50. Ussing, N. V. Geology of the Country around Julianehaab, Greenland. Medd. om Grønl. Bd. 38. Købh. 1912.

51. Wager, L. R. Geological Investigations in East Greenland. Part 1. General Geology from Angmagsalik to Kap Dalton. Medd. om Grønl. Bd. 105, no. 2. Købh. 1934.

52. - \& Deer, W. A. Geological Investigations in East Greenland. Part 3. The Petrology of the Skaergaard Intrusion Kangerdlugssuaq, East Greenland. Medd. om Grønl. Bd. 105, no. 4. Købh. 1939.

53. Walker, Fr. \& Davidson, Ch. A Contribution to the Geology of the Faeroes. Trans. Roy. Soc. Edinburgh. Vol. 58. 1936.

54. Washington, H. S. Deccan Traps and Other Plateau Basalts. Bull. Geol. Soc. of America. Vol. 33. Washington 1922.

Whittard, W. F. (1930). Vide Wordie \& Whittard.

55. Winkler, Cl. Zur Zusammensetzung des Eisens von Ovifak in Grönland und der bituminösen Kohle (des Kolms) aus der cambrischen Formation Westergötlands. Ofv. af K. Vet.-Akad. Förh. no. 7. Stockholm 1901. 
56. Wisemann, J. D. H. A Contribution to the Petrology of the Metamorphic Rocks of East Greenland. Quart. Journ. Geol. Soc. Vol. 88. London 1932.

51. Wolff, F, von. Der Vulkanismus. Bd. 2, Teil 2. 1. Stuttgart 1931.

58. Wordie, I. M. \& Whittard, W. F. A Contribution to the Geology of the Country between Petermann Peak and Kjerulf Fjord, East Greenland. Geol. Magazine, Vol. 67. London 1930.

59. Vrba, K. Beiträge zur Kenntniss der Gesteine Süd-Grönlands. Sitz.ber. der K. Akad. d. Wissensch. 1 Abt. Febr.-Heft, Bd. 69. Wien 1874.

60. Wöhler, F. Analyse des Meteoreisens von Ovifak in Grönland. Nachrichten v. d. K. Gesellsch. d. Wissenschaften u. d. G.-A. Univ. zu Göttingen. 1872 .

61. Wöhler, F. Bemerkungen über das grönländische Gediegen Eisen. Neues Jahrb. f. Min., Geol. u. Pal. Stuttgart 1879. 


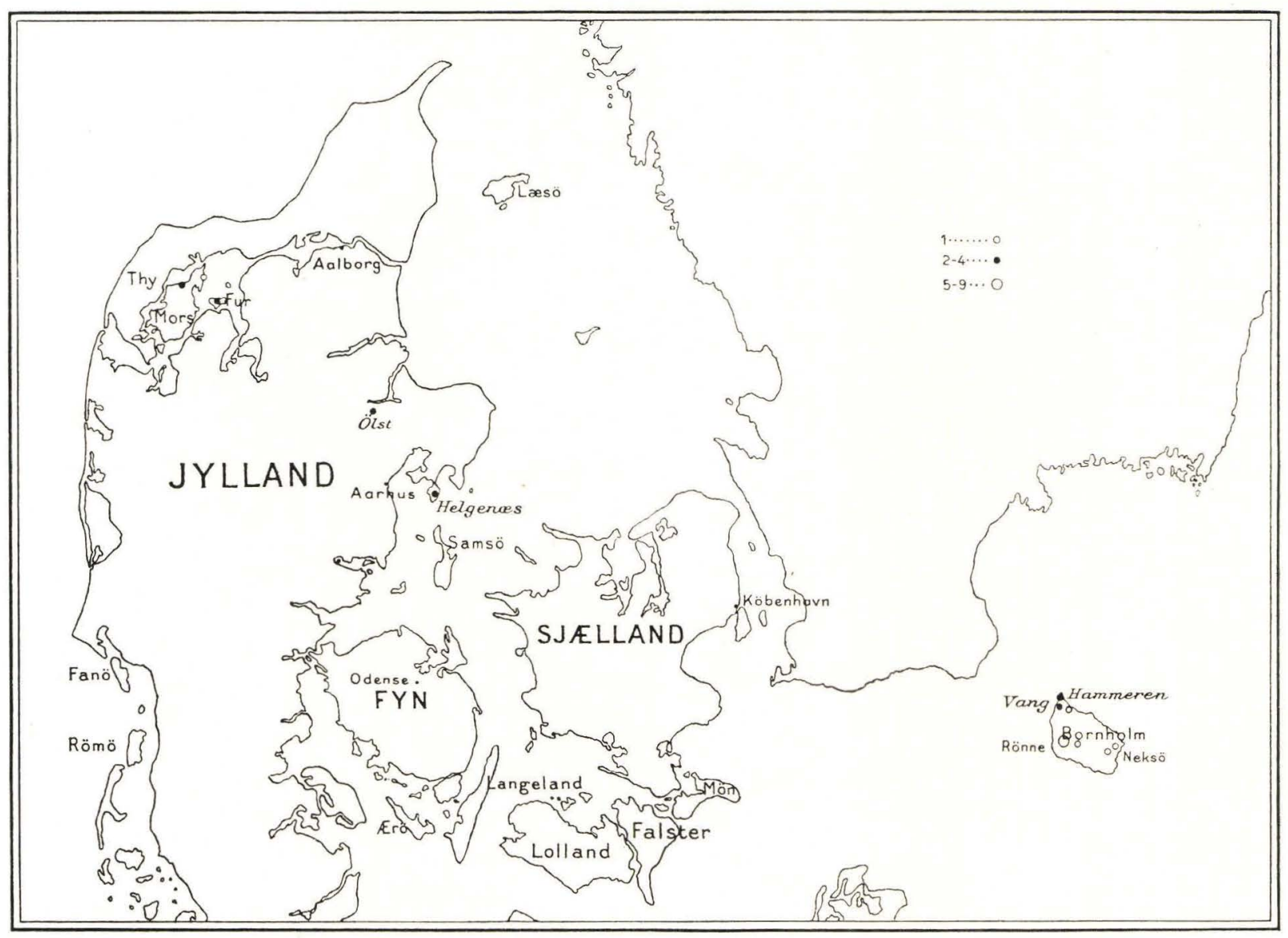

$\Xi$

Fig. 1. Map of Denmark. Letters give the number of analyses. '(Smallest dots = cities: Kobenhavn, Odense, Aarhus and Aalborg). 


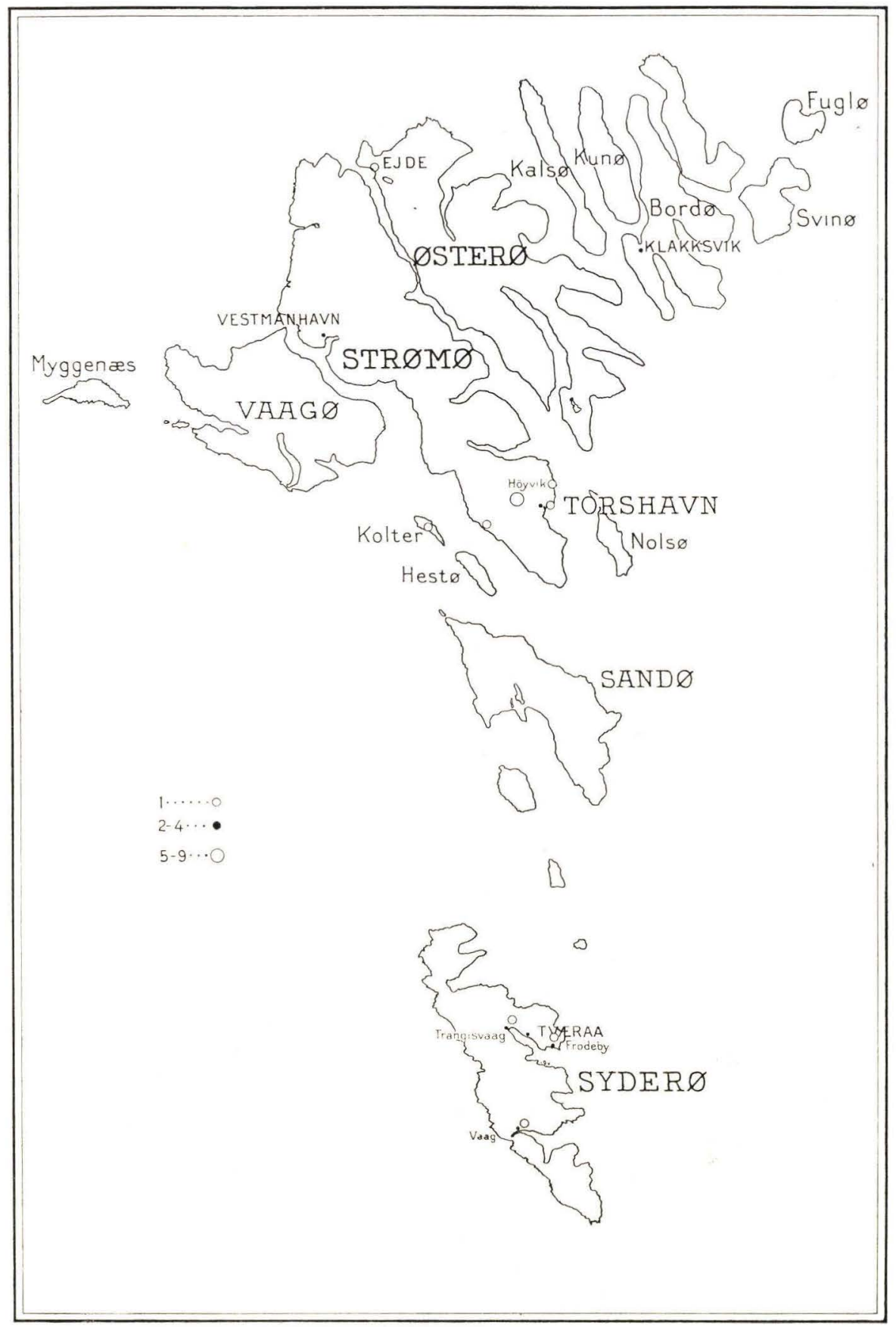

Fig. 2. Map of the Faeroes.

Letters give the number of analyses.

(Smallest dots $=$ towns and villages, Klakksvik f. inst.). 


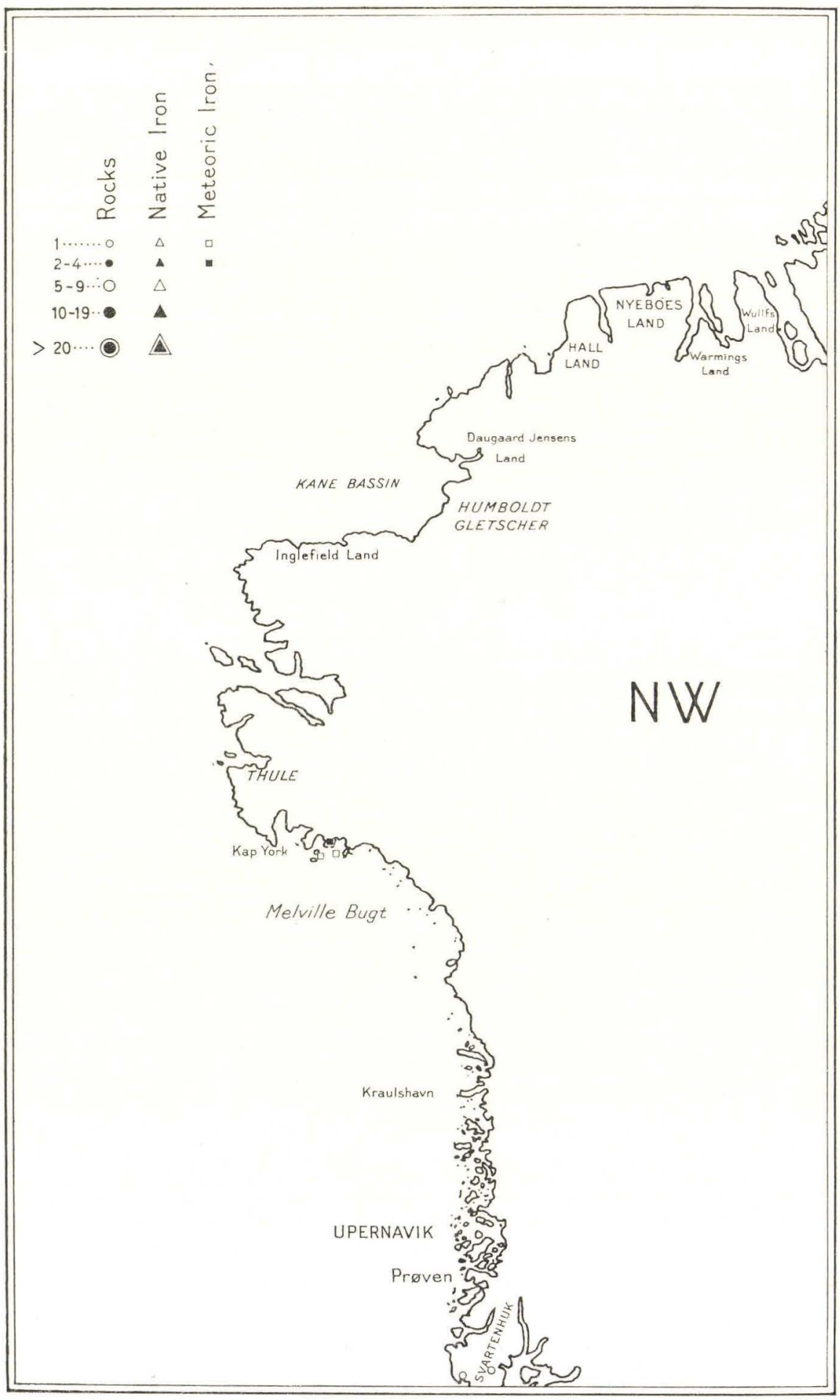

Fig. 3. Map of Greenland, northwestern part. (Continued on fig. 4). Letters give the number of analyses. 


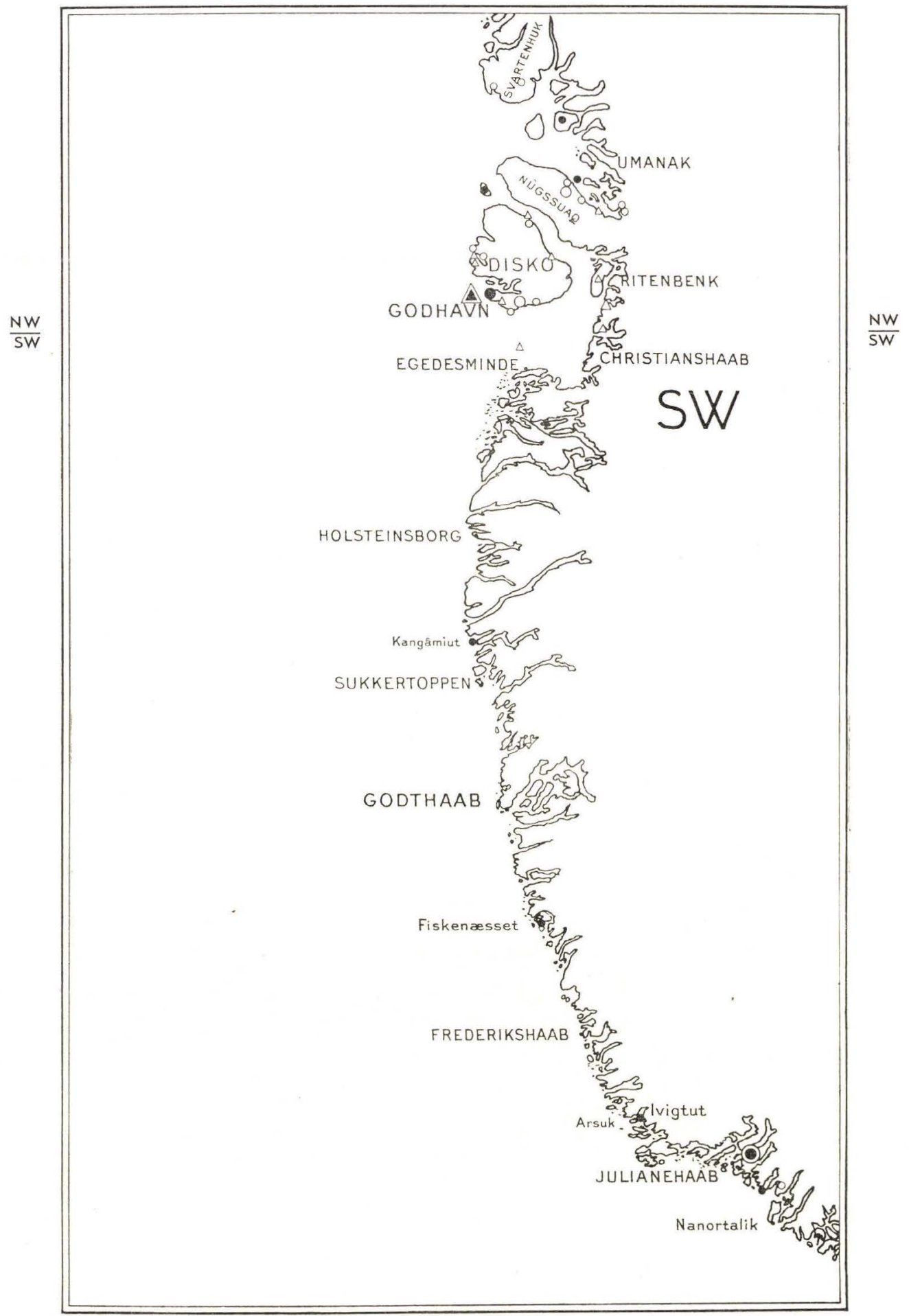

Fig. 4. Map of Greenland, southwestern part. (Legend on fig. 3). 


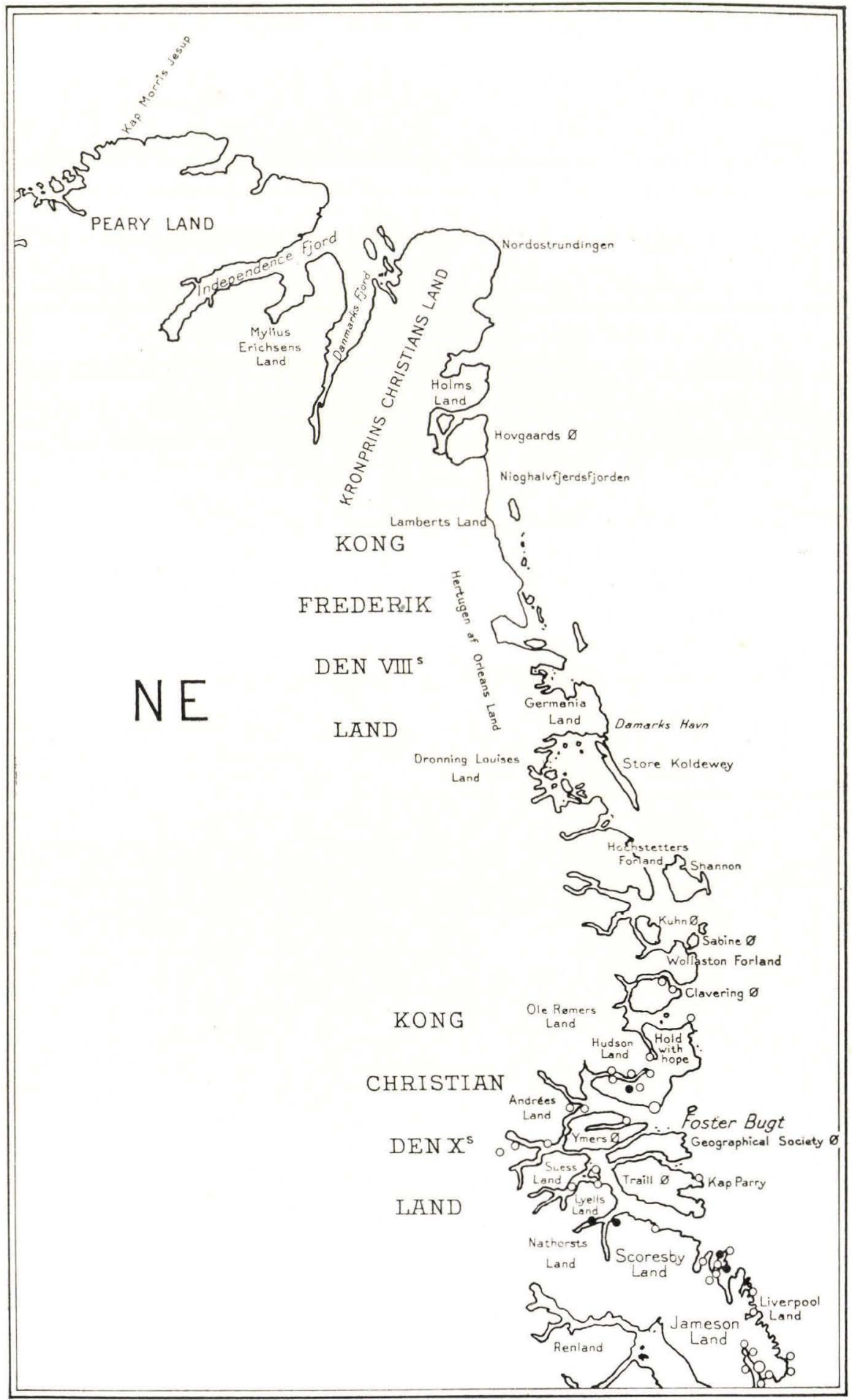

Fig. 5. Map of Greenland, northeastern part.

(Legend on fig. 3). 


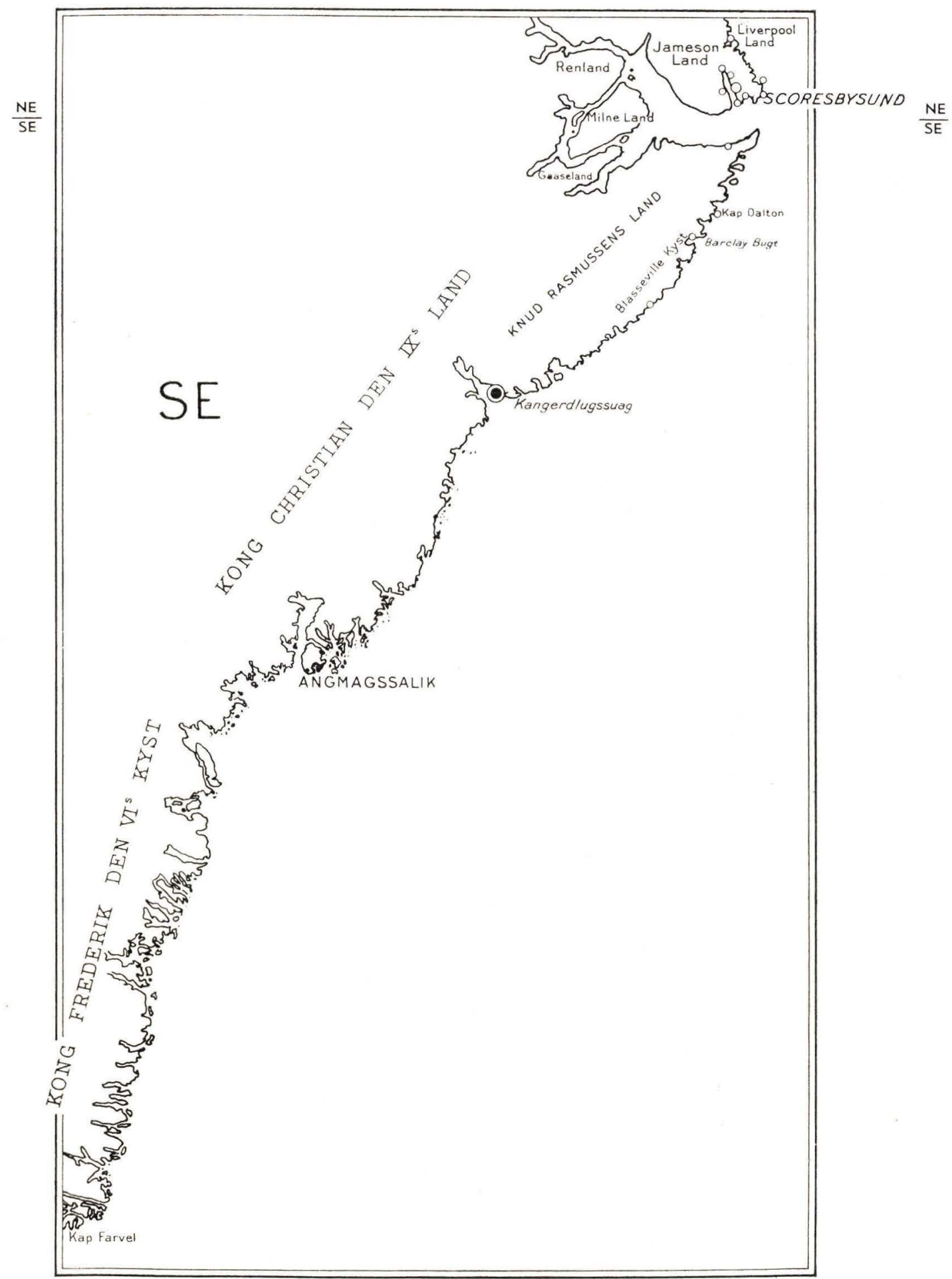

Fig. 6. Map of Greenland, southeastern part. (Legend on fig. 3). 